\title{
STUDY OF ACTIVE PARTICLES IN HETEROGENEOUS MEDIA
}

\author{
Dissertation \\ zur Erlangung des mathematisch-naturwissenschaftlichen \\ Doktorgrades \\ "Doctor rerum naturalium" (Dr.rer.nat) \\ im Promotionsprogramm ProPhys \\ der Georg-August University School of Science (GAUSS)
}

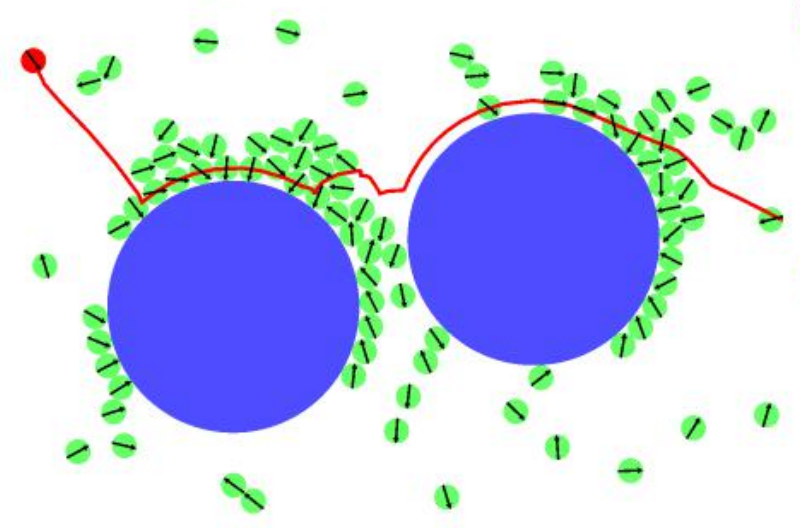

vorgelegt von

Zahra Mokhtari

aus Teheran, IRAN

Göttingen, 2018 
- Dr. Annette Zippelius

Institut für Theoretische Physik, Georg-August-Universität Göttingen

- Prof. Dr. Reiner Kree

Institut für Theoretische Physik, Georg-August-Universität Göttingen

MEMBERS OF THE EXAMINATION BOARD:

REVIEWERS

- Prof. Dr. Annette Zippelius

Institut für Theoretische Physik, Georg-August-Universität Göttingen

- Prof. Dr. Reiner Kree

Institut für Theoretische Physik, Georg-August-Universität Göttingen

FURTHER MEMBERS OF THE EXAMINATION BOARD

- Dr. Claus Heussinger, Institut für Theoretische Physik, Georg-August-Universität Göttingen

- Prof. Dr. Stefan Klumpp Institut für Nichtlineare Dynamik , Georg-August-Universität Göttingen

- Prof. Dr. Sarah Köster Institut für Röntgenphysik, Georg-August-Universität Göttingen

- Dr. Marco G. Mazza, Dynamik komplexer Fluide, Max Planck Institute for Dynamics and Self-Organization 
Dedicated to my parents,

Leila and Javad 

In this thesis I present simulation-based studies of systems of selfpropelled particles in heterogeneous media. I consider the interaction of particles with planar walls, single spherical obstacles or arrays of randomly distributed obstacles. Active particles with different propulsion mechanisms and different interactions with each other and the environment have been known to exhibit interesting universal phenomena; however, in conducting a generic theory explaining such phenomena we still require further investigation of different types of active systems. The aim of our studies in this thesis is to shed some light on the emergent behavior of individual or large collections of active particles with repulsive excluded volume interactions and linear propulsion in the presence of environmental heterogeneities.

First, I describe the behavior of single active particles in the vicinity of a simple environmental constraint: a planar wall. It is shown how the activity increases the tendency of the particles to move along the wall and spend long times in its vicinity. These results are consistent with the behavior of a large class of biological and synthetic active particles, reported previously. The distribution of the residence time on the wall is found and its dependence on different parameters is explored numerically.

Then I introduce spherical large obstacles to the system and extend our observations to this case. It is illustrated how active particles tend to reside longer on the obstacles as they flatten, and also how the residence times are affected by our model parameters, as compared to the case of a planar wall.

Next, I go on to study the collective behavior of active particles in the presence of large obstacles. The accumulation and crystallization of active particles around the obstacles are characterized: an interesting phenomenon that has been previously found in different active systems with repulsive interactions only.

I further describe a particular phenomenon of collective rotation of active particles around the obstacles. Given the purely repulsive interactions of particles with themselves and the obstacle, the absence of any active torque on the particles, and the lack of any aligning or synchronizing mechanism between the particles, such huge rotating aggregates of particles is not a trivial state of the system. I explore the origin of such rotations and using simple arguments explain why they occur. Our suggested mechanism for driving the rotations also describes some of their important properties such as the increase of 
their angular velocity as the rotations build up, and the scaling of the total torque driving the rotating crystals by their mass.

Finally, I present some results on the behavior of active systems in crowded environments. Increasing the crowdedness, the diffusion becomes non-Gaussian and slow. The decrease of the diffusion constant with the obstacle density is a function of the activity. The effect of activity on the particle's exploration of the cages, made by the many obstacles, is further investigated in this thesis. 


\section{PUBLICATIONS}

Some discussions and figures in chapter 4 have appeared previously in the following publication:

[1] Zahra Mokhtari, Timo Aspelmeier, and Annette Zippelius. "Collective rotations of active particles interacting with obstacles."

In: EPL (Europhysics Letters) 120.1 (2017), p. 14001. URL: http: //stacks.iop.org/0295-5075/120/i=1/a=14001. 

So how do you go about teaching them something new?

By mixing what they know and what they don't know.

Then when they see vaguely in their fog something they recognize, they think "Ah, I know that." And then it's just one more step to "Ah, I

know the whole thing."

And their mind thrusts forward into the unknown and they begin to recognize what they didn't know before and they increase their powers of understanding.

- Picasso, in Life with Picasso by Francoise Gilot and Carlton Lake

(Nelson, London, 1965)

\section{ACKNOWLEDGEMENTS}

Completing my doctoral studies would be far more difficult or even impossible, if it were not for some people.

My supervisor, Prof. Annette Zippelius, has been a great lead and an insightful teacher to me. Her perseverance in constantly questioning our model and our results not only guided the project, but also developed this valuable spirit in me: not to give up my questions. I am grateful for all her support and flexibility with me, and for having the experience of working with the powerful woman that she is.

I would also like to thank Dr. Timo Aspelmeier for our fruitful collaboration, and his remarkable patience and help in our long meetings. For all the situations when I gave up solving the equations and he came up with a smart solution: I am indebted and inspired by his unfailing optimism and persistence.

I am also grateful to my co-supervisor Prof. Reiner Kree for his reliable guide and his extremely instructive courses.

Many thanks go to my friends in the institute: I certainly had a great time sharing the office with Matthias Grob and enjoyed a lot from our discussions about physics. I would like to thank Stephan Filor and Timo Fischer, two of my oldest friends in Göttingen, for all the joyful memories we have. Getting to know many interesting people, during my stay on the 4 th floor of the institute, has been an unforgettable nice experience for me. Claus Heußinger, Salvatore Manmana, Till Kranz, Trisha Nath, Benjamin lenz, Florian Spreckelsen, Henry Amuasi, Shibu Saw, Florian Sohn, Kristof Harms, Thomas Köhler, Veronica Chappa, Silke Möbius, and Max Reyer, thank you for being around! 
Special thanks to Frau Schubert and Katrin Glormann, who have been unbelievably patient and helpful with sorting out all my forms and letters, and of course to Kati Oldenburg for taking care of the 4 th floor. I also appreciate Jürgen Holm's efforts on handling the computers!

I am indeed more than grateful to my husband, Ehsan Irani, who has been also a friend and colleague to me. I appreciate our inspiring discussions about my work, his constant helps, specially with running my simulations on HOOMD, and his support for me in the most frustrating situations.

My deepest gratitude goes to my family. I never forget how enthusiastic my mother was when I was lecturing her about a particular physics problem that I had learned at school, or how my father used to lead me through step-by-step solution of seemingly complicated physics problems. I owe my achievements to their inexhaustible support for me all along my life. 


\section{CONTENTS}

I INTRODUCTION I

1.1 Active particles ............... I

1.2 Biological and artifitial examples .......... 3

1.3 Modeling of active motion . . . . . . . . . . 5

1.4 Interaction with environmental geometry . . . . . . 6

1.4.1 Interaction with walls . . . . . . . . 7

1.4.2 Capture of active particles by obstacles . . . . . 7

1.4.3 Extended landscape of obstacles ........ 9

1.5 Collective behaviour and pattern formation ...... 10

1.5.1 Phase separation and crystallization ...... 10

1.5.2 Collective rotation of active particles . . . . . 12

1.6 Thesis overview . . . . . . . . . . . . . 13

2 MODEL AND METHODS 15

2.1 Model . . . . . . . . . . . . . . . . 15

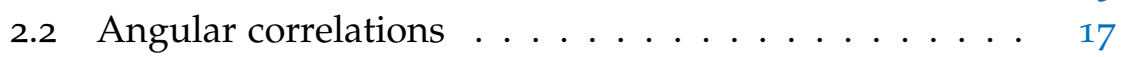

2.3 Analytical approximations . . . . . . . . . . . . 19

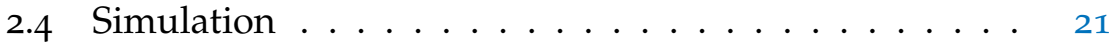

2.4.1 Implementation of obstacles . . . . . . . . 22

2.4 .2 Measurements. . . . . . . . . . . . 22

3 SElf-Propelled Particles NeAR SURfaCes 27

3.1 Residence on a flat wall . . . . . . . . . . 27

3.2 Interaction with obstacles $\ldots \ldots \ldots \ldots$

4 COLlective Rotations 39

4.1 Accumulation ..................... 39

4.2 Crystallization . . . . . . . . . . . 42

4.3 Spontaneous rotations . . . . . . . . . . 47

4.3 .1 Observation . . . . . . . . . . 48

4.3.2 Derivation of the rotations . . . . . . . 51

4.3 .3 Phase diagram ............... 56

5 CROWDED ENVIRONMENT 61

5.1 Active Lorentz model . . . . . . . . . . . . . 6 61

5.2 Transport characterization . . . . . . . . . . 62

5.3 Scaling behavior ................. 64

5.4 Exploration of cages ............. 66

6 CONCLUSION 75

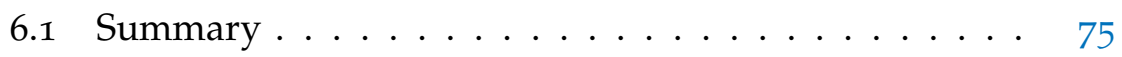

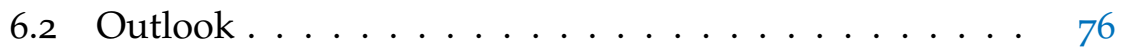

A APPENDIX 79

A.1 Finite size scaling . . . . . . . . . . . 79

BIBLIOGRAPHY 
Figure 1.1 Run-and-tumble motion of a wild type E. coli. The plotted trajectory is a projection of the bacterium's track in three dimensions. According to [4] the cell swims along straight lines for long times, then stops and tumbles, and then runs again. The tumbling time is much smaller than the run time. Reprinted by permission from [Springer Nature]: [Bacterial behaviour, H. C. Berg], Copyright (1975). . . . . .

Figure 1.2 An exhibition of polar order in a school of fish. Photo is taken by the author. . . . . . . . . . .

Figure 1.3 The rotation of an asymmetric microgear immersed in a bacterial solution. The yellow circle is to help tracking the rotation. Figure is from [57]. . . . . .

Figure 1.4 Demonstration of the mechanism through which the asymmetric shape of the gear results in the trapping of self-propelled particles in the concave corner and induces a finite torque. Figure is from [57].

Figure 1.5 System of active particles in the presence of pinned obstacles, simulated in [62]. Blue and red disks correspond to obstacles and particles respectively. The gray lines show the trajectories over a period of time. Reprinted figure with permission from [C. Reichhardt and C. J. Olson Reichhardt, Phys. Rev. E 90, 012701, 2014.] Copyright (2014) by the American Physical Society. . . . . . . . . . . . .

Figure 1.6 (a) Cluster formation in a suspension of colloidal Janus particles [71]. The indicated particle by the black arrow leaves the cluster as its orientation points away from it (left), and gets replaced by another particle (right). (b) Demonstration of self-trapping: An active particle on the cluster's boundary is trapped until its orientation changes due to rotational diffusion and points outwards. Reprinted figure with permission from [Ivo Buttinoni, et al.,Phys. Rev. Lett. 110, 238301, 2013.] Copyright (2013) by the American Physical Society. . . . . . . . . . . . II 
Figure 1.7 The static structure factor, $S(\mathbf{k})=\frac{1}{N}\left\langle\sum_{i j} e^{i \mathbf{k} . \mathbf{r}_{i j}}\right\rangle$, of a large cluster in simulations of [69]. By increasing the activity (Péclet number $\mathrm{Pe}$ ), the liquid-like isotropy changes to a sixfold-symmetry, indicating the establishment of crystalline order within the cluster. Reprinted figure with permission from [G. S. Redner, et al.,Phys. Rev. Lett. 110, 055701, 2013.] Copyright (2013) by the American Physical Society.

Figure 1.8 Rotation of a crystal composed of eight T. Majus bacteria [83]. (a)-(b)-(c) are separated by 0.17 s. (d)(f) is the result of analytical calculations which governs the dynamics of particles assuming an effective inter-particle force. Reprinted figure with permission from [A. P. Petroff, et al.,Phys. Rev. Lett. 114, 158102, 2015.] Copyright (2015) by the American Physical Society. . . . . . . . . . . . . .

Figure 2.1 Comparison of (top) the mean square displacement and (bottom) $\frac{\langle\mathbf{v}(\mathbf{t}+\tau) \cdot \mathbf{n}(\mathrm{t})\rangle}{\langle\mathbf{v}(\mathrm{t}) \cdot \mathbf{n}(\mathrm{t})\rangle}$, which measures the fidelity of the velocity to the initial orientation, in different systems with $x=y=10 \ldots \ldots \ldots$

Figure 2.2 Comparison of the exact value for $\langle\cos \phi(t) \cos \phi(0)\rangle$ derived from Eq. 2.15 (blue) and our approximation based on Eq. 2.17 (red). In the top figure $y=1$ and in the bottom one $y=0.1 \ldots \ldots$. . . . . . I9

Figure 2.3 A comparison of the results produced by our own program, "Kugeln", and HOOMD. As we can see in this figure a proper choice of corresponding parameters can generate equivalent systems, while HOOMD is much faster specially in dense systems due to its parallel computing. . . . . . . . . . . . 22

Figure 2.4 Comparison of theory with simulation results for different values of $y$. The packing fraction is 0.01 to reduce the rate of collisions and hence have a better agreement between theory and simulations. . . .

Figure 2.5 Mean displacement along the initial orientation. This quantity converges to a constant value as the displacement in future times loses correlation with the initial orientation. . . . . . . . . .

Figure 2.6 MSD for packing fraction $\phi=0.01$ and different activities. The more active a system is, the longer the initial ballistic regime lasts and the larger the translational diffusion coefficient becomes. . . . . . Translational diffusion coefficient in systems with $E_{t}=0.05$, and different $y$. The solid line represents our theoretical prediction for $\mathrm{D}_{t}$ (Eq. 2.37) and the bullets represent our simulation results. . . . . . 26 
Figure 3.1 Demonstration of the incident angle, $\theta_{i}$, in collisions with a wall. . . . . . . . . .

Figure 3.2 Distribution of the residence time of a particle on a wall, given different ranges of the incident angle. The measurement is over roughly 1000 residence

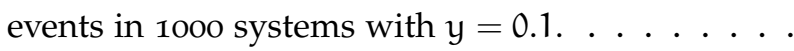

Figure 3.3 Distribution of the residence time of a particle with $y=0.1$ and $\left|\omega_{i}\right|<0.2$ on a wall. . . . . . . .

Figure 3.4 Distribution of the residence time of a particle with $y=0.1$ and $\left|\omega_{i}\right|<0.2$ on a wall, measured for $N_{f}$

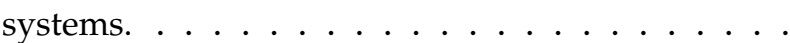

Figure 3.5 Distribution of the residence time of a particle with $y=0.1$ on a wall for different ranges of $\left|\omega_{i}\right|$. Note that according to Eq. 2.25, the average magnitude of $\omega_{i}$ is about 0.224 when $y=0.1$. This explains why we have poor statistics as $\omega_{i}>0.5$. . . . . .

Figure 3.6 Distribution of the residence time of a particle with $y=0.1$ on a wall for different ranges of the incident angle $\theta_{i}$. The long exponential tail of the distribution shifts vertically downwards as the angle between the incident orientation and the normal to the wall increases. . . . . . . . . . . .

Figure 3.7 Average of the residence time on a flat wall, given the incident angle $\theta_{i}$. The average is over 500 sys-

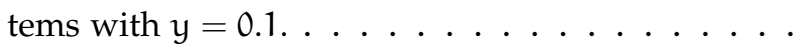

Figure 3.8

$\left\langle\tau_{\text {res }}\right\rangle$ as a function of $\theta_{i}$ and $\omega_{i}$ for $y=0.1$ (top) and $y=10$ (bottom). In active systems, this quantity changes only subtly with the initial angular velocity, but decreases considerably as the incident

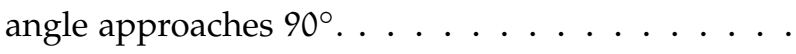

Figure 3.9 Distribution of the residence time of a particle with $y=0.1$ and different $x$ on a wall. The residence times on a planar wall are independent of $x$ and determined by the dynamics of the angle only. . .

Figure 3.10 The average residence time on a wall as a function of $y$. This quantity diverges at zero $y$ (high activity) in the form of a power-law. . . . . . . . . .

Figure 3.11 The characteristic time scale $\tau_{0}$, computed by fitting an exponential $\exp \left(-\tau_{\text {res }} / \tau_{0}\right)$ to $\mathrm{P}\left(\tau_{\text {res }}\right)$, as a

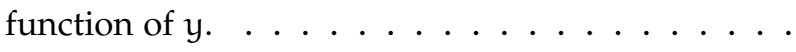

Figure 3.12 Distribution of the distance between two furthest points touched by an active particle on the wall during a residence event, for two different ranges of incident angle. . . . . . . . . 34 
Figure $3.13\left\langle\mathrm{~d}_{\text {res }}\right\rangle$ as a function of $\theta_{i}$ and $\omega_{i}$ for $y=0.1$ (left) and $y=10$ (right). In active systems this quantity changes only subtly by the initial angular velocity, but decreases considerably by enlarging the incident angle, similar to the average residence time (Fig. 3.8). . . . . . . . . . . . . . .

Figure 3.14 Comparison of the distribution of the residence time on a wall and on disk-like obstacles of different radii in systems with $y=0.1 \ldots \ldots 36$

Figure 3.15 Distribution of the residence time on anstacle with radius $R_{o} / R_{p}=10$ in systems with different $y$. Number of bins vary between 20 to 5 for $y=0.001$ to $y=1000$, and there are in average 400 points in each bin. . . . . . . . . . . .

Figure 3.16 Distribution of the residence time on abstacle with radius $R_{o} / R_{p}=10$ in systems with different $x$. The distribution changes with $x$, unlike the case of a flat wall. . . . . . . . . . . . .

Figure 3.17 Trajectory of an active particle with $x=100$ (left) and $x=0.1$ (right). Speedy particles (associated with large $x$ ) cover longer distances tangent to the obstacle's circumference in bouncing off the surface and hence slip off the obstacle quicker as compared to slower particles. . . . . . . . . . . . . .

Figure 3.18 Distribution of the number of successive collisions with an obstacle that a particle experiences during its residence on the obstacle. For systems with $y=0.1$ and $R_{o} / R_{p}=10$, the average number of successive collisions decreases dramatically by increasing x. . . . . . . . . . . . . 38

Figure 3.19 Average residence time of particles with $y=0.1$ on an obstacle with $R_{o} / R_{p}=10$ versus different incident angles. Normal incidents correspond to the longest residence times, similar to the case of a flat wall (Fig. 3.7). . . . . . . . . . . . .

Figure 4.1 Snapshot of the system with $\phi_{p}=0.08, R_{p} / R_{o}=$ 0.1 and $y=0.001$ (left), $y=10$ (right). Active particles, in contrast to passive ones, form living aggregates around the obstacles. . . . . . . . . . . Figure 4.2 Pair correlation function $(\Delta=0.5)$ of systems with $\phi_{\mathrm{o}}=\phi_{\mathrm{p}}=0.08$ and different levels of activity. The first, second and third peaks correspond to different layers of particles accumulated around obstacles at distances approximately equal to $R_{\mathrm{o}}+$ $R_{p}, R_{o}+3 R_{p}$, and $R_{o}+5 R_{p}$ from the obstacles. . . 
Figure 4.3 Height of the first peak of the pair correlation function versus $y$ for systems in Fig. 4.2. This quantity shows a crossover from the maximum number of particles that can be placed on the circumference of an obstacle in the active regime, to the value expected in a homogeneous fluid in the passive regime. 40

Figure 4.4 Obstacle-particle pair correlation function in systems with $y=0.001, R_{p} / R_{o}=0.1, \phi_{o}=0.08$ and $\phi_{p}=0.2$ (top), $\phi_{p}=0.4$ (bottom). Increasingly more layers of particles accumulate around the obstacle by increasing the packing fraction. . . . . . . Figure 4.5 Obstacle-particle pair correlation function in systems with $y=0.001, \phi_{p}=0.3, R_{p}=1$ and different obstacle radius $R_{o}$. The $x$-axis is divided by $R_{p}+R_{o}$ to compare the first peaks of the two curves. The accumulation is enhanced by enlarging the obstacle. 42

Figure 4.6 Development of crystals around the obstacles in a system with $\phi_{\mathrm{o}}=0.08, \phi_{\mathrm{p}}=0.3, \mathrm{R}_{\mathrm{p}} / \mathrm{R}_{\mathrm{o}}=0.1, \mathrm{x}=$ $0.1, y=0.001$ : figure in the left exhibits the initial configuration and figure in the right shows the configuration at some time in the steady state. . . . 43

Figure 4.7 A snapshot of a system with $\phi_{\mathrm{o}}=0.08, \phi_{\mathrm{p}}=0.4, \mathrm{R}_{\mathrm{p}} / \mathrm{R}_{\mathrm{o}}=$ $0.1, x=0.1, y=0.001$. The aggregates on the obstacle exhibit crystallization with hexagonal patches separated by grain boundaries. . . . . . . . . . . 44

Figure 4.8 The structure factor calculated for particles in the crystal of Fig. 4.7. . . . . . . . . . . 44

Figure 4.9 Snapshot of a system exhibiting a crystalline cluster around an obstacle (top) and the corresponding Delaunay triangulation of the system (bottom). Red particles in the triangulated picture, are vertices with all edges smaller than $2.1 R_{p} \ldots \ldots \ldots \ldots \ldots \ldots$

Figure 4.10 A crystalline cluster of particles formed around an obstacle $\left(R_{\mathrm{o}}=10 R_{\mathrm{p}}\right)$. Particles are colorcoded according to their activity given by Eq. 2.27 from red ( $c=1$, or perfect alignment of velocity and orientation) to blue $(\mathrm{c}=-1$, or antialignment). . . . . . . . . . . . Figure 4.11 Number of the particles within shells of radius $r$
and thickness $R_{p}$ around an obstacle, averaged over all obstacles, for a system with $\phi_{\mathrm{o}}=0.08, \phi_{\mathrm{p}}=$ $0.4, R_{p} / R_{o}=0.1, x=0.1, y=0.001 \ldots \ldots \ldots$ 
Figure 4.12 Growth, dissolution, and re-growth of the crystalline structure around the obstacle for a system with $\phi_{p}=$ $0.3, y=0.001$. (a) to (d) represent the system's configuration at different times during a single run. . $\quad 48$

Figure 4.13 Formation of a CW rotating crystal from (a) to (d). Particles are color-coded according to the orientation of their velocities: red corresponds to $\mathrm{CW}$, and blue corresponds to CCW motion around the obstacle. . . . . . . . . . . . .

Figure 4.14 The direction of angular velocity is color-coded. There is no particular order in this quantity in the rotating crystals. This is expected, as collisions do not affect $\omega \ldots \ldots \ldots \ldots \ldots$

Figure 4.15 A CW rotating growing crystal of particles around an obstacle of size $R_{o}=10 R_{p}$. The activity of particles is color-coded: red and blue correspond to $c=1$ and -1 respectively. Particles on the back of the rotating crystal tend to move almost along their orientations, whereas particles on the front are mostly pushed by the crystal opposite to their orientations. ................ 51

Figure 4.16 Gradient of local activity in two different rotating crystals, averaged over 80 snapshots. $y=0.001$ and $\phi$ is in radians. . . . . . . . . . . . . .

Figure 4.17 Demonstration of a non-rotating crystal in a system with $\phi_{p}=0.3, y=0.01$. Color-coding is based on the activity of particles. . . . . . . . . . .

Figure 4.18 Gradient of activity in the crystal shown in Fig. 4.17. The local activity is homogeneous through the entire non-rotating crystal. . . . . . . . . . . 52

Figure 4.19 Formation of a CW rotating crystal. Particles are color-coding according to their $\mathbf{v}_{\phi}$ : the spectrum from red to blue corresponds to $\mathrm{CW}$ to $\mathrm{CCW}$ motion around the obstacle respectively. Particles marked in green are to help visualizing the rigid body rotation of the cluster from snapshot a to d. . . . . . Figure 4.20 Sketch of a sample trajectory of a particle in two successive collisions with a model rough moving wall. . . . . . . . . . . . . . .

Figure 4.21 Left: $N_{ \pm}$versus time in several clusters. Red arrows indicate the time when $\frac{\mathrm{N}_{+}-\mathrm{N}_{-}}{\mathrm{N}}>0.03$; we mark the onset of rotation by this time. Right: Rotational velocity of the clusters, $\Omega \times 10^{3}$, corresponding to the left columns. Data from simulations are compared to the result of integration of Eq. 4.10 (dashed lines). . . . . . . . . . . . . . 55 
Figure 4.22

Figure 4.23

Figure 4.24

Figure 4.25

Figure 4.26

Figure 4.27

Figure 5.1

Figure 5.2

Figure $5 \cdot 3$

Figure $5 \cdot 4$
A: $\mathrm{N}_{+}-\mathrm{N}_{-}$versus total number of particles $\mathrm{N}$ in a cluster as it grows, observed in a system with $\mathrm{y}=$ $0.001, \phi_{p}=0.3$; inset: the torque $M$ as a function of $\mathrm{N}$; B: growth of $\mathrm{N}_{+}$and $\mathrm{N}_{-}$with time; C: Change of $\Omega$ with time; both (B and $C$ ) for the same cluster as in $\mathrm{A}$. The onset of the rotation is marked by the red arrow. . . . . . . . . . . . . . . Distribution of angular velocity of particles around the obstacle. $\phi_{p}=0.4$. The dashed line fits a Gaussian to the red curve. . . . . . . . . . . . . . Average magnitude of the angular velocity of the crystal around the obstacle, $\Omega$, as a function of particle activity and packing fraction. . . . . . . . Snapshot of a system at $\phi_{p}=0.7$ and $y=0.0001$, which corresponds to $1-\mathrm{c}=2.5 \times 10^{-5}$. At such high $\phi_{p}$ collective rotations are hampered by other clusters. . . . . . . . . . . . . . Snapshot of a system at $\phi_{p}=0.7$ and $y=0.1$, which corresponds to $1-c=2.5 \times 10^{-2}$. No crystalline cluster forms around the obstacle. . . . . . Demonstration of possible phases at different values of activity and packing fraction. Explanation about the boundaries between different phases can be found in the main text. . . . . . . . . . . . Distribution of the displacement of active tracers during a time interval $\tau=10000$ at different density of obstacles. The markers correspond to the simulation data points and the solid lines represent the fitted curves to the data points. Distributions are normalized and the fitted functions are normal distributions with standard deviation of the corresponding data points. . . . . . . . . . . . The non-Gaussian parameter versus time for different obstacle density. This quantity diverges at the critical point $\phi=0.22 . \ldots \ldots \ldots \ldots$ Mean square displacement of active tracers $(y=$ $0.1)$ in systems with different obstacle density $\phi$. The minimum system size corresponds to $\phi=0.16$ and is $L=450$. The dashed line $\left(\sim \tau^{0.65}\right)$ is to compare the growth of the MSD at the critical point with ref. [113]. . . . . . . . . . . . . Fraction of active particles $(y=0.1)$ that have moved less than $d=100 R_{o}$ in time interval $\tau$, for different packing fractions. . . . . . . . . . . . 65 
Figure 5.5 Similar curves as the ones in Fig. 5.4, with the $x-$ axis being re-scaled by the diffusion coefficient of the corresponding system. All the curves collapse on the top of each other. . . . . . . . . .

Figure 5.6

Figure $5 \cdot 7$

Fraction of particle that have moved less than $d=$ $50 R_{o}$ in active systems $(y=0.1)$ with different obstacle density. . . . . . . . . . . . . . Fraction of particle that have moved less than $d=$ $50 R_{o}$ in passive systems $(y=10)$ with different obstacle density. . . . . . . . . . . . . .

Figure $5.8 \quad \tau^{*}$, the time that $\mathrm{Q}(\tau, \mathrm{d})$ (given in Fig. 5.6 and 5.7) drops to 0.6 , as a function of the distance to the critical point for an active and passive system. $\tau^{*}$ appears to diverge in the form of a power-law at the critical density for both passive and active systems. The dashed line corresponds to $\tau^{*} \sim \epsilon^{-0.9}$. . . . .

Figure 5.9 The mean square displacement of tracers with different activities in obstructed systems above the percolation transition $(\phi=0.26) \ldots \ldots \ldots \ldots$

Figure 5.10 Trajectory of particles for $\Delta t \sim 300$ in systems with $\phi=0.26$ and $y=10$ (top), $y=0.1$ (bottom). Passive particles' trajectories are rather coiled as compared to active particles' extended trajectories which pass by several obstacles before re-orienting. . . . . . . Figure 5.11 Comparison of the distribution of square displacements at a time interval $t=500$ above the percolation threshold in two active and passive systems: $\phi=0.26, \mathrm{~L}=350 \ldots \ldots \ldots \ldots \ldots$

Distribution of square displacements of particles divided by their mean square displacement $(\bar{\Delta})$ in two active and passive systems. . . . . . . . . Figure 5.13 Squared radius of gyration of the trajectories of tracer particles during a time interval $\tau$ for systems with $\phi=0.26$ and $L=350 \ldots \ldots \ldots . \ldots 70$

Figure 5.14 Sample trajectories of particles in a system with $y=$ $0.1, \phi=0.4, N_{p} / N_{o}=100 / 5000, R_{p} / R_{o}=0.4$. For a particle to obstacle size ratio of $R_{p} / R_{o}=0.4$, the critical density is about $\phi=0.4 \ldots \ldots \ldots$

Figure 5.15 Obstacle-particle pair correlation function in systems with different activities above the percolation transition. $\phi=0.4, \mathrm{~N}_{\mathrm{p}} / \mathrm{N}_{\mathrm{o}}=100 / 5000, \mathrm{R}_{\mathrm{p}} / \mathrm{R}_{\mathrm{o}}=$ $0.4, \mathrm{~L}=198.166$. Active particles rather move in the vicinity of the obstacles, whereas passive ones are indifferent about them. . . . . . . . . . . 72 
Figure 5.16 Number of collisions with obstacles in systems above the percolation transition versus $y$. This number is per particle and during one unit of time. Each marker corresponds to a system similar to Fig. 5.15 with different $y \ldots \ldots \ldots \ldots \ldots$. . . . . . 72

Figure 5.17 Distribution of the distance to the closest obstacle, in a time window before reaching the steady state. $\mathrm{N}_{\mathrm{p}} / \mathrm{N}_{\mathrm{o}}=100 / 5000, \mathrm{R}_{\mathrm{p}} / \mathrm{R}_{\mathrm{o}}=0.4, \phi=0.4, \mathrm{~L}=$

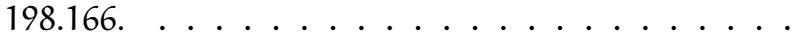

Figure 5.18 Distribution of the distance to the closest obstacle, in a time window in the steady state. System parameters are similar to Fig. 5.17. . . . . . . . .

Figure 5.19 Average distance of particles to the closest obstacle, versus $y$ in systems above the percolation threshold. This quantity reveals a crossover from moving almost tangent to the obstacles in active systems, to wandering in the middle of the cages in passive ones. 74

Figure 6.1

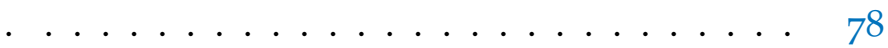

Figure A.1 $\quad \tau^{*} \epsilon^{\mu}$ as a function of $L / \epsilon^{-v}$ for different values of $\mu$ and $v$. Around $\mu=0.5, v=1.5$ the data points seem to scale power-law and then saturate at small and large values of $\mathrm{L} / \mathrm{e}^{-v}$ respectively, although the scaling is poor. . . . . . . . . . . Figure A.2 $\quad \tau^{*} \epsilon^{\mu}$ as a function of $L / \epsilon^{-\nu}$ for different values of $\mu$ and $v$. This figure is similar to Fig. A.1, but with higher resolution around $\mu=0.5, v=1.5 \ldots .8$. 81 


\section{I ACTIVE PARTICLES}

What are we talking about, when we talk about active particles?

The notion "active", used by physicists, is introduced to contrast "passive" which refers to particles with no motion of their own. A floating dust speck in water moves passively around, solely affected by the solvent's properties. The Brownian motion of the speck, run by random collisions with the surrounding molecules, is an equilibrium phenomenon well studied ever since it was first reported by Brown in 1827 and later theorized by Einstein (1905), Smoluchowski (1906), and Langevin (1908). An active particle, in contrast to the floating dust speck, owes its motion to self-propulsion: it extracts energy from the environment to move in a dissipative medium. With this basic definition, living systems provide an excellent example of active matter: They all consist of components that convert ambient or stored energy into systematic motion. In fact the study of active systems is built on the early attempts of the physicists to understand the motion of living systems. Maybe one of the earliest steps (if not the earliest) towards this understanding is the introduction of the notion of "persistent random walk" by Fürth [2] in describing the motion of biological agents in a fluid, based on his experiments. Later, the invention of the tracking microscope by Howard C. Berg and his prominent works on modeling the motion of Escherichia coli with the random walk theory [3] built an important part of the theoretical basis of this new field of study. He described the trajectories of E. coli (see Fig. 1.1) as long straight runs (persistent motion of the bacterium along the same direction) separated by tumbles (change of the direction) [4]. Purcell's inspiring paper[5] on the importance of shape and swimming mechanisms of microswimmers, is also certainly considered an insightful piece of work which has, together with other works, further enriched the groundwork of our today's knowledge of active matter.

Living systems, as the examples of active matter, are not limited to microorganisms. In fact many of the early models presented to study self propelled particles, aimed at simulating the motion of macroscopic animals [6, 7]. Fish in the ocean, birds in the sky, and animal herds on land are examples of such self-propelling systems. All these systems are different in shape, size, propulsion mechanism, interaction with each other and with their environments, but are similar with respect to some characteristics which distinguish them from passive systems. 
Most importantly, they are all out of equilibrium systems. The con-
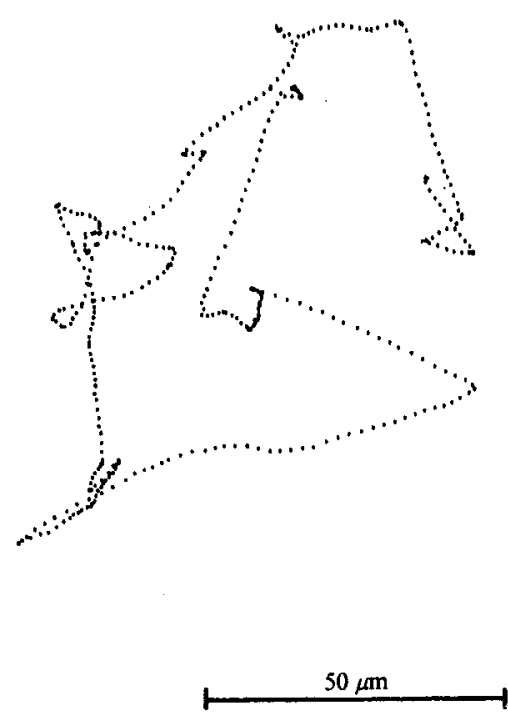

Figure 1.1: Run-and-tumble motion of a wild type E. coli. The plotted trajectory is a projection of the bacterium's track in three dimensions. According to [4] the cell swims along straight lines for long times, then stops and tumbles, and then runs again. The tumbling time is much smaller than the run time. Reprinted by permission from [Springer Nature]: [Bacterial behaviour, H. C. Berg], Copyright (1975).

stant uptake and dissipation of energy in such systems drives them out of equilibrium and hence even in the absence of any interactions with each other or the medium, they exhibit non-trivial behavior [8, 9]. Novel states of matter arise in interacting active systems, which are absent in their equilibrium counterparts. Take a simple dense suspension of bacteria for example: the fluid can exhibit unusual rheological properties [10], exhibit turbulent motion [11], the bacteria can selforganize in complex patterns [12], swim in circles near surfaces [13] and form a solid-like biofilm [14]. These are only some of such emerging collective effects in active systems that can not be predicted from the dynamics of the individual particles.

Another important property shared by all self-propelled particles is the persistence of their trajectories, as mentioned earlier. There is a correlation between successive steps of an active particle. In other words the particle remembers its orientation for some time, during which it travels a finite distance (the persistence length) along its orientation.

Active particles have attracted increasing attention in the last few decades as they allow physicists to explore the involved physical processes in evolutionary biology. Transport (diffusion or mixing), loco- 
motion, and taxis (interaction with chemicals, light, etc.) are some basic features of life a general theoretical description of which is hard to achieve, given the overall complexity of living matter. However, the development of new technologies and the theoretical efforts on quantifying the stochastic trajectories of animals have improved our understanding of their dynamics, which not only increases our control over the biological processes in the body or our environment, but also may shed some light on more fundamental questions regarding life.

In the following sections, I introduce different types of active particles and their motility mechanisms. Then different models that have successfully described distinct aspects of active systems will be explained. I will then move on from the individual dynamics of isolated particles, and introduce the studies on the interaction of an active particle with its environment. We will learn about the history of the research on interacting active particles with different realizations of heterogeneities in their media. Eventually the case of many active particles and the emergent phenomena that arise from their collective behavior will be discussed. In the last section I give an overview of the contents of this thesis.

\subsection{BIOLOGICAL AND ARTIFItiAL EXAMPLES}

Active particles are ubiquitous in nature. Bacteria $[15,16]$, protozoa [17, $18]$, sperm cells $[19,20]$, and algae [21, 22] are some examples of microswimmers. Their self-propulsion is generated by flagella or cilia, flexible lash-like appendages to cell bodies composed of several microtubules. The flagella have rotary, snake-like, or stroke-like motion in different animals. The helical motion of the flagella on Escherichia coli for example, generates a thrust that pushes the cell from the rear (pushers). Whereas a Chlamydomonas, with its two flagella on the front, pulls itself forward through a breaststroke-like motion ("pullers"). Pushers and pullers generate different flow fields around them which need to be carefully calculated when the hydrodynamic interactions between them is the subject of study.

How does a sperm cell find the egg in its journey through the female cervix? How do bacteria find food? How does a dental plaque form? How does a C. elegance move in a porous medium like soil? How do parasites like Trypanosoma manage to swim in the bloodstream and adapt their motion to the density of cells in blood [23]? Why does flagella beating in algae cells like Chlamydomonas synchronize? Or on the macroscopic scale, how do the fish or birds communicate in order to form fish schools or bird flocks, where they move collectively and aligned (see Fig. 1.2)? Can we describe the physical properties of social interactions in real world swarms and flocks? 
These are some of the questions that physical scientists have been

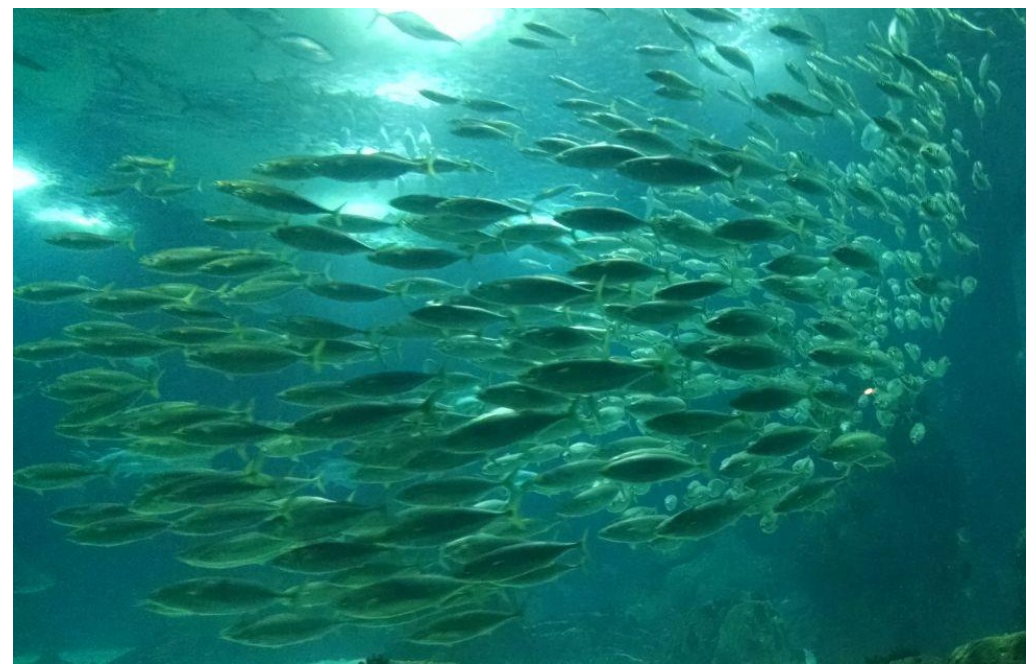

Figure 1.2: An exhibition of polar order in a school of fish. Photo is taken by the author.

trying to answer in the last few decades. To this end, minimal mathematical models that capture some of the emergent behavior in the real systems are presented. Some of these models will be introduced in the next section.

Furthermore, to realize some of the models suggested for the dynamics of active particles and reproduce the behavior of motile biological organisms, various types of artificial self propelled particles have been designed in laboratories. A famous example for such systems in the micro scale is synthetic Janus colloids (named after the two-faced Roman god), where dielectric colloids are coated with two different thin layers of catalytic materials on the two halves [24]. Immersing these colloids in a $\mathrm{H}_{2} \mathrm{O}_{2}$ solution, the two halves decompose $\mathrm{H}_{2} \mathrm{O}_{2}$ into $\mathrm{H}_{2} \mathrm{O}$ and $\mathrm{O}_{2}$ differently and thus generate a local concentration gradient that eventually leads to self-diffusiophoresis. The colloid is hence self propelled along its orientation (the vector connecting the two caps) which itself is subject to thermal rotational diffusion.

Some other examples of artificial active systems are active water droplets (see $[25,26])$, semi-flexible rods in a rotating magnetic field (see [27, 28]), and vibrated polar granular disks [29] or rods [30].

Synthetic self propelled particles not only facilitate our understanding of real active systems, but also serve as a playground to enhance our general knowledge of out of equilibrium systems. Besides, they have direct valuable applications in health care and environment [3133]. 


\subsection{MODELING OF ACTIVE MOTION}

As briefly mentioned in the previous section, since the end of the twentieth century different models have successfully described or even predicted the observed phenomena in active systems. Among all active particles in nature or laboratories microswimmers, small objects that swim in viscous fluids, generate flow fields around them and exhibit an explicit hydrodynamic coupling with the fluid. Models that consider only the observable effects of net motion, and not the explicit hydrodynamic interactions, may not then be as successful in describing the systems of microswimmers. Nevertheless, there are numerous cases where hydrodynamics interactions do not play an important role: Some of the examples are dry active systems [34], or highly crowded media [35] where steric collisions may dominate longrange hydrodynamic effects. Below I will introduce some of these models which ignore the details of the swimming in the microscopic level and yet are found to be considerably powerful in describing a wide range of active systems.

Inspired by the Brownian motion of passive particles, an "active Brownian particle" (ABP) moves with a constant velocity $v$ along its "orientation", $\mathbf{n}=(\cos \phi, \sin \phi)$, which is subject to rotational diffusion. The dynamics, in the absence of interactions, is run by the following stochastic differential equations:

$$
\left\{\begin{array}{l}
\dot{x}=v \cos \phi+\sqrt{2 \mathrm{D}_{\mathrm{T}}} \zeta_{x} \\
\dot{y}=v \sin \phi+\sqrt{2 \mathrm{D}_{\mathrm{T}}} \zeta_{\mathrm{y}} \\
\dot{\phi}=\sqrt{2 \mathrm{D}_{\mathrm{R}}} \zeta_{\phi},
\end{array}\right.
$$

where $\zeta_{x}, \zeta_{y}$, and $\zeta_{\phi}$ are white Gaussian noise with zero mean and correlation $\delta(t)$. $D_{T}$ and $D_{R}$ represent the translational and rotational diffusion coefficient of a passive particle respectively; they are functions of the temperature of the fluid and particle's geometry only and are obtained for a passive particle. Collisions between such particles is usually assumed to be repulsive. By increasing the self-propelling speed $v$, the particle performs a persistent motion along its orientation at small times. At long times the motion is diffusive due to the rotational diffusion, with a systematic enhancement of the effective translational diffusion coefficient with increasing $v$ [36]. Active Brownian particles can describe the dynamics of Janus particles fairly well.

The "run-and-tumble" dynamics, describing the motion of some bacteria and certain types of algae [37] which move in a sequence of "runs" interrupted by Poisson-distributed "tumbles" (rapid change of direction), is another extensively used model in active matter. It has been shown that the long-time diffusion properties of run-and- 
tumble particle (RTP) are equivalent to those of active Brownian particles, albeit their short-time dynamics are different $[38,39]$.

There are several more general models that consider underdamped dynamics, finite-time correlations in the orientational dynamics, active torque [40, 41], or many other scenarios for the one-particle motion in active systems. For example the dynamics of colloidal particles in a bacterial bath has been regarded as an Ornstein-Uhlenbeck process, where the magnitude of the colloid's velocity is no more constant, but multiple collisions with the bacteria gradually change its direction and amplitude [42]. Or if instead, the dynamics of the orientation is regarded as an Ornstein-Uhlenbeck process, the finite-time correlations in the orientational dynamics can affect the particle's diffusivity relative to the cases of memoryless change of orientation in $\mathrm{ABP}$ and RTP [43].

In the presence of other particles, the mutual interaction of particles can not only change the single particle motion, but also give rise to unexpected collective dynamics. That makes the adjustment of the particle-particle interaction, an important part of a model.

Various types of interactions, mainly hydrodynamic and steric (excludedvolume or soft), have been worked out in the computational study of active systems. A particularly widespread phenomena in active systems is the alignment of neighboring particles, which can lead to flocking or swarming. This suggests the introduction of aligning interactions between particles. Hydrodynamic interactions [44, 45], steric interaction between self propelled rods [46] or even disks [47], or an explicit adaptation of the orientation to the average direction of motion of the particles in their local neighborhood [7] are some known possibilities to induce particle-particle alignment and describe the collective behavior of different classes of active systems to a very good extent.

\subsection{INTERACTION WITH ENVIRONMENTAL GEOMETRY}

Active particles, biological or synthetic, hardly ever live in homogeneous environments; they rather move in the presence of physical boundaries. Can we describe the interaction of single active particles with walls or obstacles using simple physical rules? Can we simulate the natural phenomena arising from the collective behavior of active particles near physical boundaries? Have motile biological systems developed certain moving mechanisms to navigate better in complex environments like highly crowded cytoplasm or porous structure of soil?

These are some of the primary questions regarding the motion of self propelled particles in heterogeneous media. As a short first step in 
answering some of these questions, below I focus on the history of studies on the role of heterogeneity in active systems and show how the interplay of activity and interactions with boundaries can give rise to phenomena that are absent in passive systems.

\subsubsection{Interaction with walls}

The study of interaction of active particles with walls can be traced back to 1963, when Rothschild observed the accumulation of sperm cells near the walls of a container. A similar effect was observed later with bacteria [48] and algae [49]. This was a peculiar phenomena exclusively observed in active systems. Passive systems at equilibrium require large attraction strength to reproduce somehow similar effects.

There have been two main explanations for these effects. One takes long-range hydrodynamic interactions into account [48]: the far-field flows generated by pusher microswimmers induce an effective attraction to the wall and a torque that tends to turn the orientation toward parallelism.

The other approach rationalizes the observed behavior based on contact interactions with the wall[50, 51]. In this picture an elongated microswimmer travels along the wall after hitting it, due to steric forces and torques exerted by the wall, until the rotational diffusion runs it away. Spherical particles have also been shown, through this approach, to exhibit long residence times traveling on flat walls due to the persistence in their motion. The gist of this mechanism is that in collisions with a wall the orientation does not change: Although the normal component of the velocity is reflected, the active force along the orientation brings it back towards the wall. Simulations based on this approach have reproduced the experimental results for E. coli and bull spermatozoa swimming near walls [50].

Several analysis and investigation of these two explanations suggest that hydrodynamics plays a minor role and contact interactions are more dominant [22] in cell-cell interactions in dilute systems and also in cell-surface interactions.

\subsubsection{Capture of active particles by obstacles}

Obstacles, being a pile of dead non-motile cells in a bacterial colony, heavy passive particles or polymers in blood, or an apparatus implemented into artificial active systems in order to guide/sort/rectify the particles' motion, can have more complex geometries than a flat wall and hence may give rise to unexpected single-particle or collective behavior. 
Similar to flat walls, active particles are expected to experience long residence times on large spherical obstacles. This has been observed in bacterial [52] and synthetic active systems [53] and the trapping times have been shown to increase with the obstacle's size and the inverse of particle's rotational diffusion constant.

In contrast to passive particles, the persistence in active particles' motion opens up the possibility of controlling their motion and harnessing their power. In the simulations done by Kaiser et al. [54] the interaction of active rods with a stationary large wedge is studied. It is found that particles can be best trapped in the wedge for an intermediate range of the wedge angle. Such trapping of self-propelled rods is also experimentally observed in systems of sperms cells [55] and artificial rod swimmers [56]. Furthermore Leonardo et al. [57] have exploited the bacterial motion to drive a microdevice: They have reported a rotation of their designed micro ratchet immersed in a bath of bacteria (Fig. 1.3). The asymmetry of the gear together with the persistence of the self propelled rods are the key points in running the rotations; the bacteria sliding towards the concave corners get trapped there and insert local torque on the gear until a tumbling event reorient and free them, whereas those orienting towards the peaks of the gear slide along the wall and escape it when they get to the top. Fig. 1.4 illustrates these two cases.

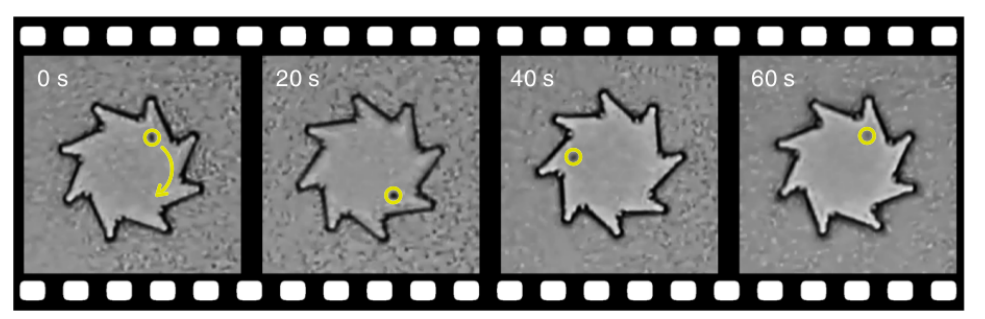

Figure 1.3: The rotation of an asymmetric microgear immersed in a bacterial solution. The yellow circle is to help tracking the rotation. Figure is from [57].

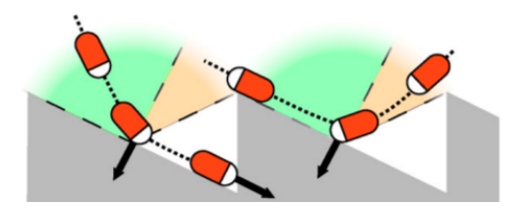

Figure 1.4: Demonstration of the mechanism through which the asymmetric shape of the gear results in the trapping of self-propelled particles in the concave corner and induces a finite torque. Figure is from [57]. 


\subsubsection{Extended landscape of obstacles}

The motion of active particles may also be highly influenced by the presence of many obstacles. Such problems are studied experimentally [58-61] and also numerically for run-and-tumble particles [62, 63], particles with aligning interactions [64] and chiral particles [65]. Below I go over some examples revealing peculiar results of the interplay of activity, inter-particle interactions, and heterogeneity.

In ref. [62] a system of run-and-tumble particles (RTP) subject to a drift force is simulated in the presence of stationary randomly distributed obstacles (Fig. 1.5). It has been shown in [62] that the transport is maximized at an optimum value of activity (the run length in RTP). For very small run length, corresponding to small activity, particles get clogged by the obstacles and the net transport is very small. As the activity is raised, the clusters behind the obstacles tend to break and let particles flow along the drift force. However at extremely long run lengths, corresponding to large activities, the transport is reduced again, due to the formation of large living crystals which facilitates the pinning of large number of particles by only a few obstacles. Cluster formation and phase separation of the system into a crystalline solid and a dilute gas is a widespread phenomenon observed in active systems and will be discussed in the next section.

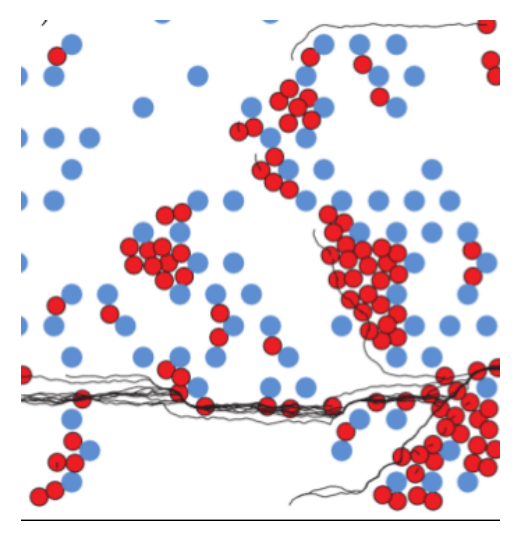

Figure 1.5: System of active particles in the presence of pinned obstacles, simulated in [62]. Blue and red disks correspond to obstacles and particles respectively. The gray lines show the trajectories over a period of time. Reprinted figure with permission from [C. Reichhardt and C. J. Olson Reichhardt, Phys. Rev. E 90, 012701, 2014.] Copyright (2014) by the American Physical Society.

Chepizhko et al. have considered in [64] a model with aligning interactions between active particles in a heterogeneous environment of obstacles. They have modeled the obstacles as series of force centers in the system which deflect the velocity vector of the approaching particles and have considered a noise term in the particle alignment 
direction. They have observed that maximal coherent motion is found for an optimum value of the noise term. Although in the absence of obstacles increasing the noise randomizes the motion and moves the system away from the state with coherent motion, the presence of obstacles hinders any coherent motion when the noise term is small. Increasing the noise to some intermediate value then facilitates particles escape from the traps and induces ordering of particles. Further increase of the noise diminishes the collective motion as expected from the obstacle-free case.

Random energy landscapes for active particles have been generated in the lab by using speckle patterns [58], or fabricated micro structures as obstacles [59-61]. In systems with purely repulsive particleobstacle interactions, the obstacles are observed to induce a slow down of motion and result in anomalous diffusion (subdiffusion) at intermediate times. Ref. [6o], realizing a random Lorentz gas that undergoes a localization transition at the void percolation threshold, provides a playground to answer questions similar to those raised in chapter 5 .

\subsection{COLLECTIVE BEHAVIOUR AND PATTERN FORMATION}

Active systems, from the scale of bacterial colonies to the scale of animal groups, exhibit similar collective phenomena such as swarming, pattern formation, turbulence, or order-disorder transitions. Ignoring the possibly psychological messages that they may transfer, a physicist's approach for understanding such collective behavior is to consider a minimal model for particles' interactions. We have reviewed in previous sections that particles with explicit or implicit aligning interactions tend to form large bundles of coherently moving particles. This is the case in Viscek-like models [7] (describing e.g. swarming of bird flocks) or the result of the combination of persistence and hard-core repulsion in active rods [30,66] (describing e.g. swarming in elongated bacterial colonies). However such collective behavior is observed in numerical and experimental studies of systems of spherical particles with no alignment rule as well. In this section I explain the out of equilibrium collective effects in several active systems and the proposed mechanisms to describe them.

\subsubsection{Phase separation and crystallization}

Spontaneous formation of clusters in the presence of attractive interparticle interactions is an old observation in systems in equilibrium. However, active systems exhibit clustering at moderate densities even when the interactions are purely repulsive. This phenomena in systems with no alignment between particles was only recently predicted 
theoretically [67-70] and realized in laboratories [71-73].

Such systems are found to exhibit an athermal clustering instability to a phase-separated regime, which can be understood in terms of a mean-field model with the propulsion velocity decreasing with the local density. In this model if the velocity decreases fast enough with the density, the homogeneous phase turns out to be unstable [67].

Such a phase separation can alternatively be explained based on the "self-trapping" of particles: Two colliding active particles, block each other for a while, due to the persistence of their motion. Collision of other particles with this small cluster leads to its growth. A particle on the edge of such a cluster needs a time $\sim 1 / D_{r}$ to re-orient and escape the cluster (see Fig. 1.6). Therefore depending on the ratio of the collision rate and the rotational diffusion $D_{r}$, the stable steady state corresponds to a homogeneous solution with small dynamical clusters, or growing clusters until the systems is separated into a large solid cluster and a dilute gas[70].

The solid phase is not static and is constantly changing its shape through exchanging particles with the diluted phase [71].

One may naively expect that activity destabilizes and ruptures the aggregates, just like thermal fluctuations do. But on the contrary, such motility-induced clustering is enhanced by increasing the activity[69] which indicates that an "effective temperature" in an equilibrium system cannot describe this effect.

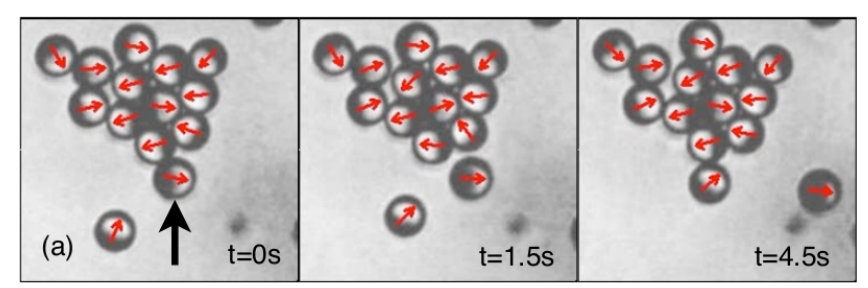

(b)

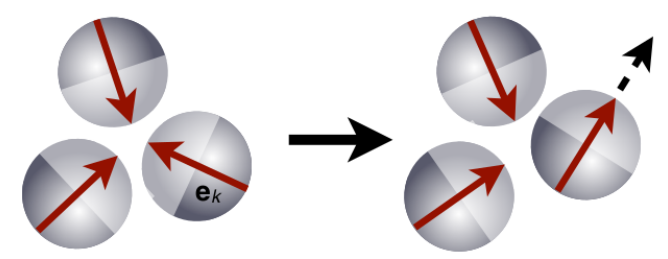

Figure 1.6: (a) Cluster formation in a suspension of colloidal Janus particles [71]. The indicated particle by the black arrow leaves the cluster as its orientation points away from it (left), and gets replaced by another particle (right). (b) Demonstration of self-trapping: An active particle on the cluster's boundary is trapped until its orientation changes due to rotational diffusion and points outwards. Reprinted figure with permission from [Ivo Buttinoni, et al.,Phys. Rev. Lett. 110, 238301, 2013.] Copyright (2013) by the American Physical Society. 
The dense phase in the phase-separated active systems mentioned above, is susceptible to crystallization [74], as expected from dense aggregates of monodisperse spheres. The sixfold-symmetry of the static structure factor of the active clusters in two-dimensional systems, indicating the hexagonal order, has been observed in computational [69, 74] (see Fig. 1.7) and experimental [71, 73, 75] studies of such systems. Such active crystals are unique, as they are held together by active forces alone, and exhibit differences from equilibrium crystals; e.g. their melting differs from their passive counterpart $[74,76]$. There are also observations of honeycomb-like clusters for flagellated marine bacteria [77] which was later explained through the generic analysis of active crystals by Löwen et al. [76, 78].

The active crystals, also known as "living crystals", are not static: They form, translate, rotate, and split constantly. Below I introduce a special dynamics exclusively observed in such active crystalline cluster.

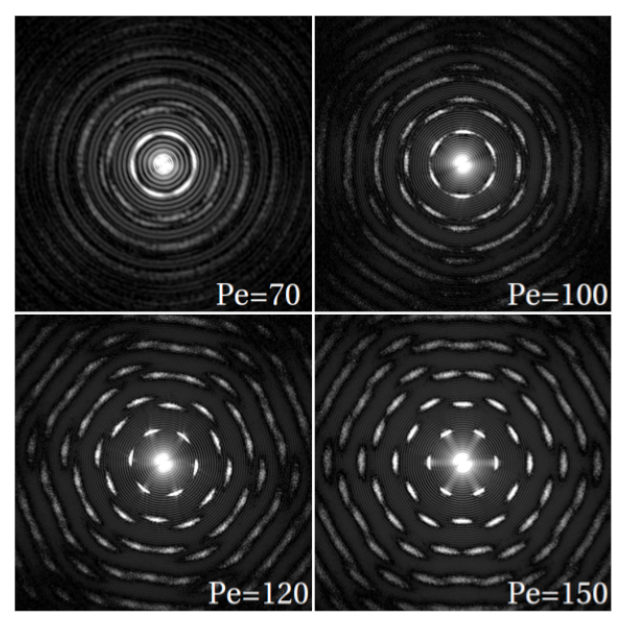

Figure 1.7: The static structure factor, $S(\mathbf{k})=\frac{1}{N}\left\langle\sum_{i j} e^{i \mathbf{k} . \mathbf{r}_{i j}}\right\rangle$, of a large cluster in simulations of [69]. By increasing the activity (Péclet number $\mathrm{Pe}$ ), the liquid-like isotropy changes to a sixfold-symmetry, indicating the establishment of crystalline order within the cluster. Reprinted figure with permission from [G. S. Redner, et al.,Phys. Rev. Lett. 110, 055701, 2013.] Copyright (2013) by the American Physical Society.

\subsubsection{Collective rotation of active particles}

Since the observation of single sperm cells' entrapment at surfaces [19], a great number of researches have been devoted to understanding the collective behavior of such cells near surfaces. A particular behavior is the organization of sperm cells into arrays of vortices near planar surfaces at high densities [20]. Such a collective rotation of several cells in a cluster around a common center has been explained based on the hydrodynamic coupling of the sperm tails [20] and re- 
produced computationally assuming inter-particle attractive and repulsive forces [79].

The study of rotating aggregates of active particles have attracted much attention ever since, and several mechanisms have been proposed to run the rotations, depending on the single particle propulsion mechanism and inter-particle forces.

Self-assembled microrotors are formed in systems of mutually attracting motile bacteria [80] due to the residual net torque exerted by the bacteria's flagella located on the surface of the cluster. Schwarz-Linek et al. [80] claim that if each of the $\mathrm{N}$ bacteria on the surface exerts a torque of magnitude $T_{0}$ on the cluster, assuming that these torques are randomly oriented, the total torque rotating the cluster is given by $T_{\text {tot }} \approx \sqrt{N} T_{0}$. Their analytical calculations, ignoring the effects of hydrodynamics, compares well with the result of their experiments.

A more drastic measurement of the rotation of active clusters suggests that in the presence of repulsive inter-particle interactions and linear self-propulsion only, dumbbellar active particles (in contrast to spherical ones) can form rotating aggregates [81, 82]. The dumbbell's orientations are essentially quenched in dense clusters due to steric interactions in the form of spirals and hence the sum of individual torques exerted by each particle in the cluster gives rise to a finite net torque.

Rotating crystals of active particles have also been observed in systems with internal torques. Experiments on T. majus bacteria [83] that rotate upon striking surfaces (see Fig. 1.8) and Janus magnetic particles that rotate in the presence of rotating magnetic fields [84] reveal collective rotation of crystalline clusters of such particles, which arises from the hydrodynamic effects leading to the synchronization of neighboring particle's rotations. Simulations of rationally driven spinners of concave shape also exhibit rotating crystals at large enough packing fraction and large active torque [41]. The gear-like shape of the particles together with the self-propelling torque gives rise to this phenomenon in [4I], explaining the rotating aggregates of rotationally-driven active particles in the absence of hydrodynamic effects.

A particular form of rotating crystals is the one we observe [85]. I will show in chapter 4 that spherical particles with repulsive interactions and linear propulsion only, can exhibit spontaneous collective rotations in the presence of large obstacles.

\subsection{THESIS OVERVIEW}

This thesis is arranged in the following chapters. In chapter 2 I introduce the model we use for active particles and through some exact calculations point out the importance of different model parameters. I 

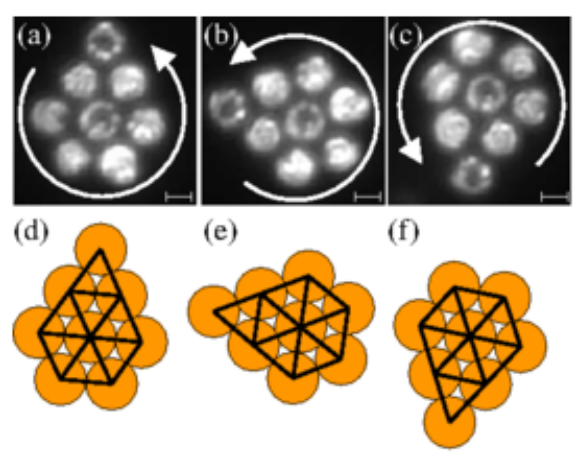

Figure 1.8: Rotation of a crystal composed of eight T. Majus bacteria [83]. (a)-(b)-(c) are separated by $0.17 \mathrm{s.}$ (d)-(f) is the result of analytical calculations which governs the dynamics of particles assuming an effective inter-particle force. Reprinted figure with permission from [A. P. Petroff, et al.,Phys. Rev. Lett. 114, 158102, 2015.] Copyright (2015) by the American Physical Society.

further explain the details of our simulation techniques and show the comparison of simulation results and exact solution of some quantities in dilute systems. In chapter 3 I explore the effect of boundaries, planar walls or large spherical obstacles, on the single particle dynamics in our active system. Active particles are found to experience long residence times on the obstacles (as predicted by the literature), the dependence of which on our model parameters and collision properties are discussed. The collective behavior of particles in the presence of large obstacles is studied in chapter 4 , where I present the crystallization of active particles on the obstacles and the new phenomenon of the crystal's systematic rotations. We develop a minimal argument to describe the rotations and find an estimation for the onset of rotations, as well as the crystal's rotating velocity. Chapter 5 focuses on the dynamics of particles in highly crowded environments. Given the tendency of active particles to linger at the surfaces, the effect of activity on the transport in such systems is not trivial. This question, or in other words the role of activity in escaping from the local cages surrounded by obstacles, is investigated in this chapter. Finally in chapter 6 I conclude and introduce part of our studies that are yet to be pursued. 
In this chapter we describe how we model active particles analytically. We further explain the implementation of our model through computer simulations. Different simulation techniques and tools that we have utilized are discussed and detailed calculation of several quantities that will be measured in this work, is given.

\subsection{MODEL}

We consider the particles in our system as hard spheres of mass $m$, moment of inertia $I$ and radius $R_{p}$ moving in a fluid in 2D. Selfpropulsion of a particle is achieved through an active force, $\mathrm{ma}_{\mathrm{d} r}$, along its "orientation". The orientation of a particle,

$$
\mathbf{n}=(\cos \phi, \sin \phi),
$$

is specified by an angle $\phi$ that is initially chosen arbitrarily and is subjected to changing due to its rotational diffusion. The motion in the fluid is both rotationally and translationally damped with drag coefficients $\alpha$ and $\gamma$ respectively. Particles undergo hard-core collisions which reflect the normal component of velocities and leave the magnitude of the translational and rotational velocity unchanged (elastic non-dissipative collisions). The orientations are untouched in the collisions as well. One can then describe our rotational dynamics as

$$
I \dot{\omega}(t)=-\alpha \omega(t)+\sqrt{v_{r}} \zeta(t),
$$

where $\zeta(t)$ is a white Gaussian noise $\left(\langle\zeta\rangle=0,\left\langle\zeta(t) \zeta\left(t^{\prime}\right)\right\rangle=\delta\left(t-t^{\prime}\right)\right)$. For a disk with uniform distribution of mass, I can be written in terms of the mass and radius as $I=\frac{1}{2} m R_{p}^{2}$. However we keep I as an independent parameter to allow for non-uniform distribution of mass which provides us with the opportunity of studying the underdamped regime as well as the overdamped (with $\mathrm{I} \rightarrow 0$ ) regime.

Translational motion can similarly be described as

$$
m \dot{\mathbf{v}}(t)=-\gamma \mathbf{v}(t)+m a_{d r} \mathbf{n}(t)+\left.m \frac{\Delta \mathbf{v}}{\Delta t}\right|_{\text {coll }} .
$$

Choosing the radius of particles $\left(R_{p}=1\right)$ and their mass $(m=1)$ as the unit of length and mass respectively, we pursue de-dimensionalization of our equations of motion. Throughout this work, we take $\mathrm{I}=1$ unless said otherwise. Given all the parameters, $\alpha, \gamma, v_{r}$, and $a_{d r}$ determining our timescale is not trivial at this point. One should note that the initial energy of the system does not set the timescale in our 
simulation, and is relaxed to the steady-state energy after adequate number of collisions.

We consider $1 / \alpha$ as our unit of time and define dimensionless velocity and angular velocity as functions of the dimensionless time $\tilde{t}=\alpha \mathrm{t}$ : $\tilde{\mathbf{v}}(\tilde{\mathrm{t}})=\frac{\mathbf{v}(t)}{\alpha R_{\mathrm{p}}}$ and $\tilde{\omega}(\tilde{\mathfrak{t}})=\frac{\omega(t)}{\alpha}$. With this definition, the derivatives with respect to the dimensionless time of these quantities are

$$
\begin{aligned}
& \frac{d}{d \tilde{t}} \tilde{\mathbf{v}}(\tilde{t})=\frac{1}{\alpha^{2} R_{p}} \frac{d \mathbf{v}(t)}{d t}, \\
& \frac{d}{d \tilde{t}} \tilde{\omega}(\tilde{\mathfrak{t}})=\frac{1}{\alpha^{2}} \frac{d \omega(t)}{d t} .
\end{aligned}
$$

We furthermore define a noise term as a function of the dimensionless time, $\tilde{\zeta}(\tilde{\mathfrak{t}})=\frac{\zeta(\mathrm{t})}{\sqrt{\alpha}}$, which is a white Gaussian noise with delta correlation in the dimensionless time:

$$
\begin{aligned}
\left\langle\tilde{\zeta}\left(\tilde{\mathrm{t}}_{1}\right) \tilde{\zeta}\left(\tilde{\mathrm{t}}_{2}\right)\right\rangle= & \frac{1}{\alpha}\left\langle\zeta\left(\mathrm{t}_{1}\right) \zeta\left(\mathrm{t}_{2}\right)\right\rangle \\
& =\frac{1}{\alpha} \delta\left(\mathrm{t}_{1}-\mathrm{t}_{2}\right) \\
& =\delta\left(\tilde{\mathrm{t}}_{1}-\tilde{\mathrm{t}}_{2}\right) .
\end{aligned}
$$

Now considering the fact that $\gamma$ and $\alpha$ both are functions of the viscosity and the geometry of particles only, we can assume that $\gamma=c \alpha$ (where $c$ is a function of the radius of particles only ${ }^{1}$ ). The above equations of motion can then be rewritten as

$$
\begin{gathered}
\frac{\partial}{\partial \tilde{\mathrm{t}}} \tilde{\mathbf{v}}(\tilde{\mathrm{t}})=-\frac{\mathrm{c}}{\mathrm{m}} \tilde{\boldsymbol{v}}(\tilde{\mathrm{t}})+\sqrt{\tilde{x}} \mathbf{n}(\tilde{\mathrm{t}})+\left.\frac{\Delta \tilde{\mathbf{v}}}{\Delta \tilde{\mathrm{t}}}\right|_{\text {coll }}, \\
\mathrm{I} \frac{\partial}{\partial \tilde{\mathrm{t}}} \tilde{\mathfrak{\omega}}(\tilde{\mathrm{t}})=-\tilde{\mathfrak{\omega}}(\tilde{\mathrm{t}})+\sqrt{\tilde{\mathrm{y}}} \tilde{\zeta}(\tilde{\mathrm{t}})
\end{gathered}
$$

where $\tilde{x}=\frac{a_{\mathrm{dr}}^{2}}{\alpha^{4} R_{\mathrm{p}}^{2}}$ and $\tilde{y}=\frac{v_{\mathrm{r}}}{\alpha^{3}}$.

We can now remove the tildes and rename the dimensionless quantities to eye-friendlier ones, considering that we take $m=1$ and $R_{p}=1$ :

$$
\begin{aligned}
\dot{\mathbf{v}}(t) & =-\mathrm{cv}(\mathrm{t})+\sqrt{x} \mathbf{n}(\mathrm{t})+\left.\frac{\Delta \mathbf{v}}{\Delta t}\right|_{\text {coll }} \\
\mathrm{I} \dot{\omega}(t) & =-\omega(t)+\sqrt{y} \zeta(t)
\end{aligned}
$$

with $x=\frac{a_{d r}^{2}}{\alpha^{4}}$ and $y=\frac{v_{r}}{\alpha^{3}}$. Hence we have shown that our model can be described by just the two parameters $x$ and $y$. This is confirmed by our simulation results. Fig. 2.1 compares quantities in several different systems with similar $x, y$. By de-dimensionalizing the $x$-axis (dividing it by our unit of time) all the curves in each figure fall on the top of each other. Detailed explanation of the simulations we used for plotting the figures below, will come in the next section.

1 Calculation of $\mathrm{c}$ in 2D is not viable through Stokes equations and one may instead consider particles as spheres bounded to move on a plane in a thin sheet. This assumption allows a finite estimation for $c$ (function of the thickness and viscosity of the sheet [86]). We take $c=1$ for simplicity. 

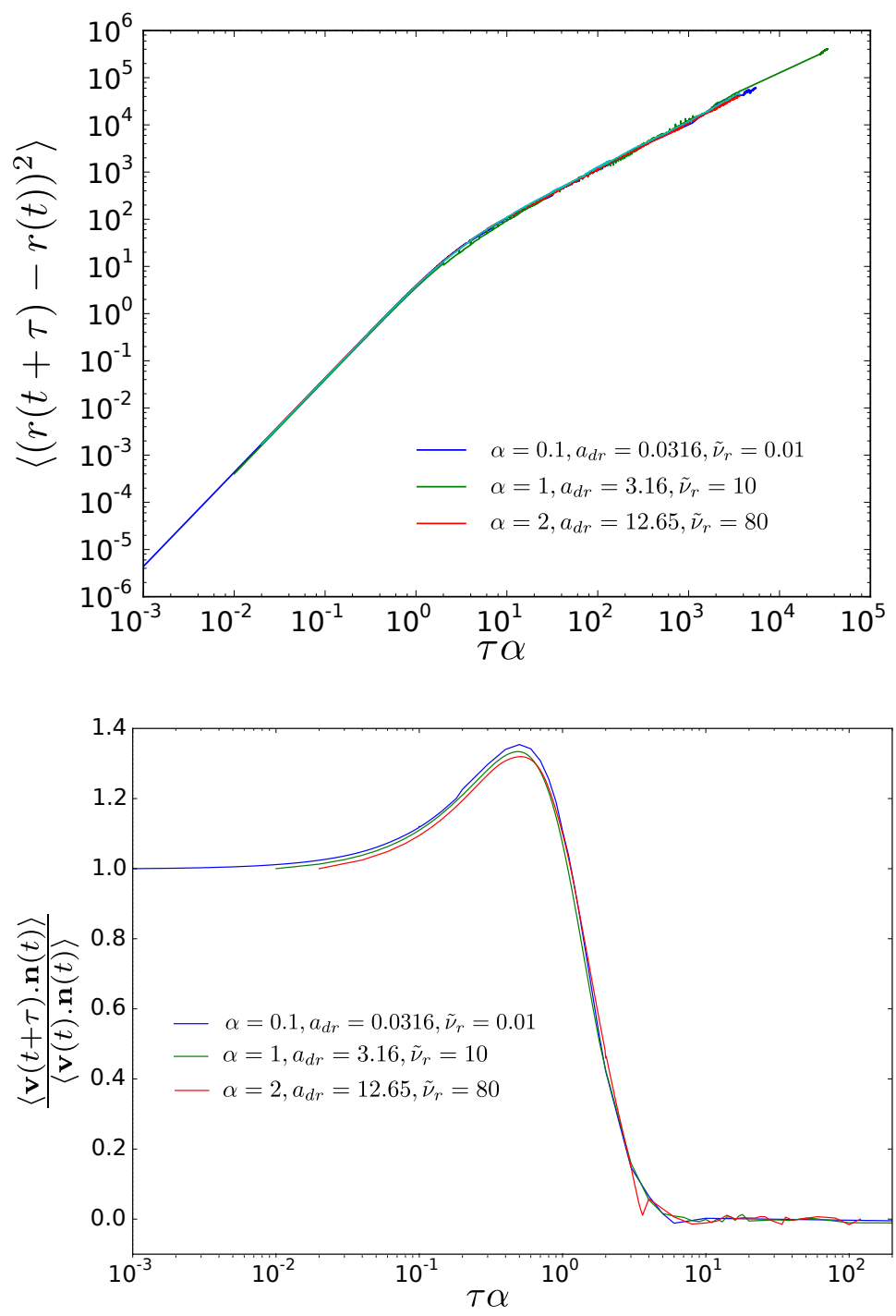

Figure 2.1: Comparison of (top) the mean square displacement and (bottom) $\frac{\langle\mathbf{v}(\mathbf{t}+\tau) \cdot \mathbf{n}(t)\rangle}{\langle\mathbf{v}(t) \cdot \mathbf{n}(t)\rangle}$, which measures the fidelity of the velocity to the initial orientation, in different systems with $x=y=10$.

\subsection{ANGULAR CORRELATIONS}

The Langevin equation for rotational motion, Eq. 2.9b, has a well known solution and predicts the observables regarding particles' orientations exactly. Namely an observable, which is essential in obtaining the velocity, translational energy or other quantities related to the displacement of particles, is the autocorrelation of the noise in 
Eq. 2.9a: $\langle\mathbf{n}(t) \mathbf{n}(t /)\rangle$. In order to calculate it, the first step is to find the mean square angular displacement (MSAD):

$$
\begin{aligned}
\left\langle(\phi(t)-\phi(0))^{2}\right\rangle & =\left\langle\int_{0}^{t} \omega\left(t_{1}\right) d t_{1} \int_{0}^{t} \omega\left(t_{2}\right) d t_{2}\right\rangle \\
& =\int_{0}^{t} \int_{0}^{t}\left\langle\omega\left(t_{1}\right) \omega\left(t_{2}\right)\right\rangle d t_{1} d t_{2},
\end{aligned}
$$

where $\omega(t)=\omega(0) e^{-t / I}+\int_{0}^{t} \frac{\sqrt{y}}{I} \zeta\left(t_{1}\right) e^{-\frac{t-t_{1}}{I}} d t_{1}$. Therefore we need to calculate $\left\langle\omega\left(t_{1}\right) \omega\left(t_{2}\right)\right\rangle$ first. In the steady state, when the initial velocities are not important any more:

$$
\begin{aligned}
\left\langle\omega\left(t_{1}\right) \omega\left(t_{2}\right)\right\rangle & =\frac{y}{I^{2}} e^{-\frac{t_{1}+t_{2}}{I}} \int_{0}^{t_{1}} \int_{0}^{t_{2}} \delta\left(t_{1}^{\prime}-t_{2}^{\prime}\right) e^{\frac{t_{1}^{\prime}+t_{2}^{\prime}}{I}} d t_{1}^{\prime} d t_{2}^{\prime} \\
& =\frac{y}{2 I}\left(e^{-\frac{\left|t_{1}-t_{2}\right|}{I}}-e^{-\frac{t_{1}+t_{2}}{I}}\right) .
\end{aligned}
$$

Using the calculated value of $\left\langle\omega\left(t_{1}\right) \omega\left(t_{2}\right)\right\rangle$ in Eq. 2.10 and noting that the second term in Eq. 2.11 vanishes in the steady state, we find that

$$
\begin{aligned}
\left\langle(\phi(t)-\phi(0))^{2}\right\rangle & =\frac{y}{2 I} \int_{0}^{t} \int_{0}^{t} e^{-\frac{\left|t_{1}-t_{2}\right|}{I}} d t_{1} d t_{2} \\
& =y\left[t-I\left(1-e^{-t / I}\right)\right] .
\end{aligned}
$$

The autocorrelation of the $x$ component of the noise in Eq. 2.9a is

$$
\begin{aligned}
\langle\cos \phi(t) \cos \phi(0)\rangle & =\frac{1}{4}\left\langle\left(e^{i \phi(t)}+e^{-i \phi(t)}\right)\left(e^{i \phi(0)}+e^{-i \phi(0)}\right)\right\rangle \\
& \approx \frac{1}{2}\left\langle e^{i(\phi(t)-\phi(0))}\right\rangle
\end{aligned}
$$

at large time t. Since $\phi$ has a Gaussian distribution,

$$
\left\langle e^{i(\phi(t)-\phi(0))}\right\rangle=e^{-\frac{1}{2}\left\langle(\phi(t)-\phi(0))^{2}\right\rangle} .
$$

Consequently we have

$$
\begin{aligned}
\langle\cos \phi(t) \cos \phi(0)\rangle & =\frac{1}{2} e^{-\frac{1}{2}\left\langle(\phi(t)-\phi(0))^{2}\right\rangle} \\
& \approx \frac{1}{2} \exp \left(-\frac{y}{2}\left[t-I\left(1-e^{-t / I}\right)\right]\right) .
\end{aligned}
$$

In the absence of collisions, having the exact value for the autocorrelation of the noise in the equation for translational motion, calculation of observables like

$$
\left\langle v_{x}(t) \cos \phi(t)\right\rangle=\int_{0}^{t} \sqrt{x} e^{-\left(t-t_{1}\right)}\left\langle\cos \phi\left(t_{1}\right) \cos \phi(t)\right\rangle d t_{1}
$$


seems plausible. However, given the complicated form of $\left\langle\cos \phi\left(t_{1}\right) \cos \phi(t)\right\rangle$ in Eq. 2.15, carrying out the integration in e.g. Eq. 2.16 is not straightforward and demands increasingly more effort for more complicated integrals. Therefore we make the following approximation:

$$
\left\langle(\phi(t)-\phi(0))^{2}\right\rangle= \begin{cases}0 & t<I, \\ y t-y I & t \geqslant I\end{cases}
$$

A comparison of the exact value and our approximation for $\langle\cos \phi(t) \cos \phi(0)\rangle$ is illustrated Fig. 2.2 for $I=1$ and $y=1$ (left) and $y=0.1$ (right). The approximation improves by decreasing $y$.
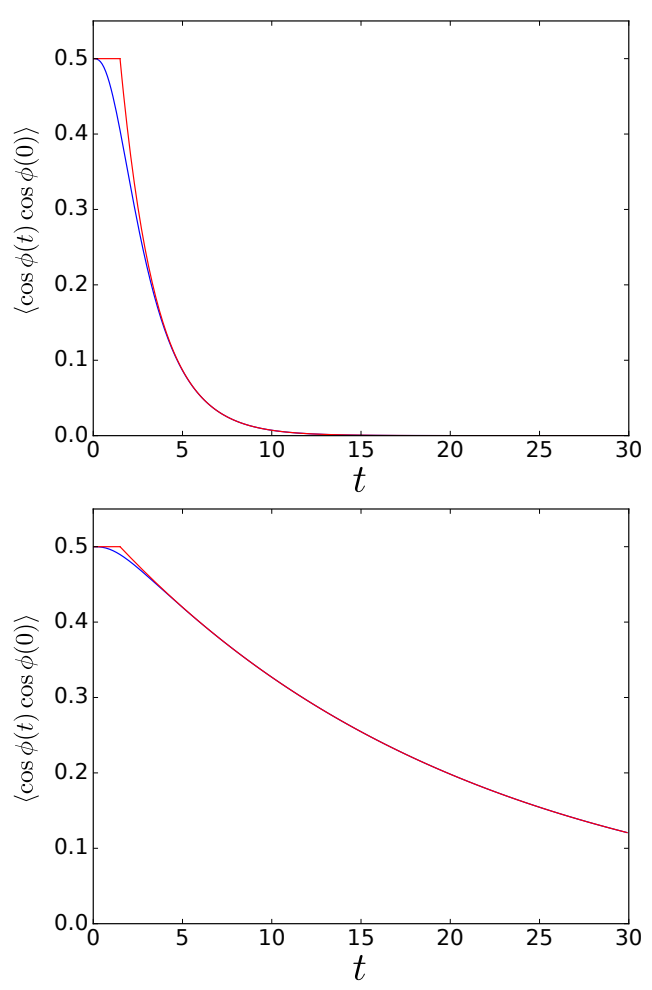

Figure 2.2: Comparison of the exact value for $\langle\cos \phi(t) \cos \phi(0)\rangle$ derived from Eq. 2.15 (blue) and our approximation based on Eq. 2.17 (red). In the top figure $y=1$ and in the bottom one $y=0.1$.

\subsection{ANALYTICAL APPROXIMATIONS}

In Dilute systems, where the collisions can be neglected in the equations of motion (Eq. 2.9a), we use the above approximation (Eq. 2.17) to find

$$
\begin{aligned}
\left\langle v_{x}(t) \cos \phi(t)\right\rangle & =\frac{\sqrt{x}}{2} e^{-t}\left[\int_{0}^{t-I} e^{t_{1}} e^{-y \frac{t-t_{1}}{2}} e^{y I / 2} d t_{1}+\int_{t-I}^{t} e^{t_{1}} d t_{1}\right] \\
& =\frac{\sqrt{x}}{2} e^{-t}\left[e^{y I / 2} e^{-y t / 2} \frac{e^{(1+y / 2)(t-I)}-1}{1+y / 2}+e^{t}-e^{t-I}\right]
\end{aligned}
$$


Hence as $t \rightarrow \infty$

$$
\langle\mathbf{v} . \mathbf{n}\rangle=\frac{\sqrt{x}}{1+y / 2}\left[e^{-I}+(1+y / 2)\left(1-e^{-I}\right)\right],
$$

Similarly $\left\langle\mathbf{v}^{2}\right\rangle$ can be analytically estimated:

$$
\begin{aligned}
&\left\langle v_{x}^{2}\right\rangle=x \int_{0}^{t} \int_{0}^{t} d t_{1} d t_{2} e^{-\left(t-t_{1}\right)} e^{-\left(t-t_{2}\right)}\left\langle\cos \phi\left(t_{1}\right) \cos \phi\left(t_{2}\right)\right\rangle \\
&=2 x \int_{0}^{t} d t_{1} e^{-\left(t-t_{1}\right)} \int_{0}^{t_{1}} d t_{2} e^{-\left(t-t_{2}\right)}\left\langle\cos \phi\left(t_{1}\right) \cos \phi\left(t_{2}\right)\right\rangle \\
&=\frac{2 x}{2} \int_{0}^{t} d t_{1} e^{-\left(t-t_{1}\right)}\left[\int_{0}^{t_{1}-I} d t_{2} e^{-\left(t-t_{2}\right)} e^{-y \frac{t_{1}-t_{2}}{2}} e^{y I / 2}+\int_{t_{1}-I}^{t_{1}} d t_{2} e^{-\left(t-t_{2}\right)}\right] \\
&=x e^{-2 t} \int_{0}^{t} d t_{1}\left[\frac{e^{y I / 2}\left(e^{2 t_{1}-I(1+y / 2)}-e^{t_{1}(1-y / 2)}\right)}{1+y / 2}+\left(e^{2 t_{1}}-e^{2 t_{1}-I}\right)\right] \\
&=x e^{-2 t}\left[\frac{e^{y I / 2-I(1+y / 2)} \frac{1}{2}\left(e^{2 t}-1\right)-e^{y I / 2} \frac{e^{(1-y / 2) t}-1}{1-y / 2}}{1+y / 2}+\frac{1}{2}\left(e^{2 t}-e^{2 t-I}\right)\right] \\
&=\frac{x}{1-y^{2} / 4}\left[e^{-I} \frac{1}{2}(1-y / 2)\left(1-e^{-2 t}\right)-e^{y I / 2}\left(e^{-(1+y / 2) t}+e^{-2 t}\right)\right. \\
&\left.\quad+\frac{1}{2}\left(1-y^{2} / 4\right)\left(1-e^{-I}\right)\right] .
\end{aligned}
$$

Hence at large $t$

$$
\left\langle\mathbf{v}^{2}\right\rangle=\frac{x}{1+y / 2}\left[\frac{y}{2}\left(1-e^{-I}\right)+1\right] .
$$

Carrying out similar calculations enables us to approximate quantities like rotational and translational diffusion coefficient, energy, and fidelity of velocity to the orientation of a particle. In the limit of $I \rightarrow 0$, when

$$
\langle\cos \phi(t) \cos \phi(0)\rangle=\frac{1}{2} e^{-\frac{y}{2} t},
$$

the result of such calculations are presented below:

$$
\begin{gathered}
\mathrm{D}_{\mathrm{r}}=\frac{\mathrm{y}}{2}, \\
\mathrm{D}_{\mathrm{t}}=\frac{\mathrm{x}}{\mathrm{c}^{2} \mathrm{y}}, \\
\left\langle\omega^{2}\right\rangle=\frac{\mathrm{y}}{2} \\
\left\langle v^{2}\right\rangle=\frac{\mathrm{x}}{\mathrm{c}\left(\mathrm{c}+\frac{\mathrm{y}}{2}\right)^{\prime}} \\
\mathrm{C}=\langle\mathbf{v} \cdot \mathbf{n}\rangle / \sqrt{\left\langle\mathbf{v}^{2}\right\rangle}=\sqrt{\frac{1}{1+\frac{1}{2 c} y}},
\end{gathered}
$$


where the translational and rotational diffusion constants are defined as $D_{t}=\lim _{t \rightarrow \infty} \frac{\Delta r^{2}}{4 \Delta t}$ and $D_{r}=\lim _{t \rightarrow \infty} \frac{\Delta \phi^{2}}{2 \Delta t}$ respectively. The latter in the list above, is the quantity which we call the "activity parameter". $1 / y$ is a measure of the activity, such that the correlation between velocity and orientation approaches its maximum value of 1 as $y \rightarrow 0$. The other control parameter, $x$, is the analogue of the temperature in a passive system.

The limit of a passive particle is obtained by taking $y \rightarrow \infty$ while keeping $\left\langle\mathbf{v}^{2}\right\rangle$ fixed. In that limit, the persistence time of the noise $(\sqrt{x} \mathbf{n})$ in Eq.2.9a goes to zero, while the amplitude increases correspondingly, resulting in white noise:

$$
\lim _{x, y \rightarrow \infty} x e^{-y|t-t /|} \sim \delta(t-t /),
$$

where $\delta(t)$ is the Dirac delta function.

\subsection{SIMULATION}

In this section I elaborate on the implementation of our model in computer simulations. Designing the heterogeneity of the medium and technical detail in carrying out measurements are further discussed in this section.

We simulate Eq. 2.2 and 2.3 through an event driven algorithm, described in [87] in a periodic square box of size $L$ with $N_{p}$ particles at a packing fraction $\phi_{p}$. The active acceleration, $a_{d r} n$, is implemented by kicks at regular time intervals $\tau_{\mathrm{dr}}=0.1 \alpha$, which changes particle's velocity by an amount $a_{d r} \tau_{d r}$ along its momentary orientation. Simultaneously the rotational velocity is changed by a random amount, $\omega_{\mathrm{d} r}$, drawn from a Gaussian with zero mean and variance $\left\langle\omega_{\mathrm{dr}}^{2}\right\rangle=v_{\mathrm{r}} \tau_{\mathrm{dr}}$. Two particle collisions are hard core elastic with no change of the orientation or angular velocity.

Our simulations in chapter 4.3, were done on graphical processing units (GPUs) with the HOOMD-blue software package [88, 89], with the kicks being substituted by a constant active force. Particles in these MD simulations experience soft interactions, namely through the conservative part of a DPD potential. The corresponding spring constant, $\mathrm{K}_{\mathrm{s}}$, is chosen large compared to the parameter $x$ (first defined in Eq. 2.9a) in order to keep the overlap of particles in collisions small $\left(\frac{\Delta x^{2}}{R_{p}^{2}}=\frac{\left\langle v^{2}\right\rangle}{R_{p}^{2}} \frac{m}{K_{s}} \ll 1\right)$ and be consistent with our assumption of hard particles. Equivalent systems are realizable by a proper choice of the corresponding parameters in the two programs. Fig. $2.3 \mathrm{com}-$ pares the mean square displacement of two similar systems simulated by our event-driven program and HOOMD. The agreement is almost perfect and the later is considerably faster as it runs in parallel and on GPU. 


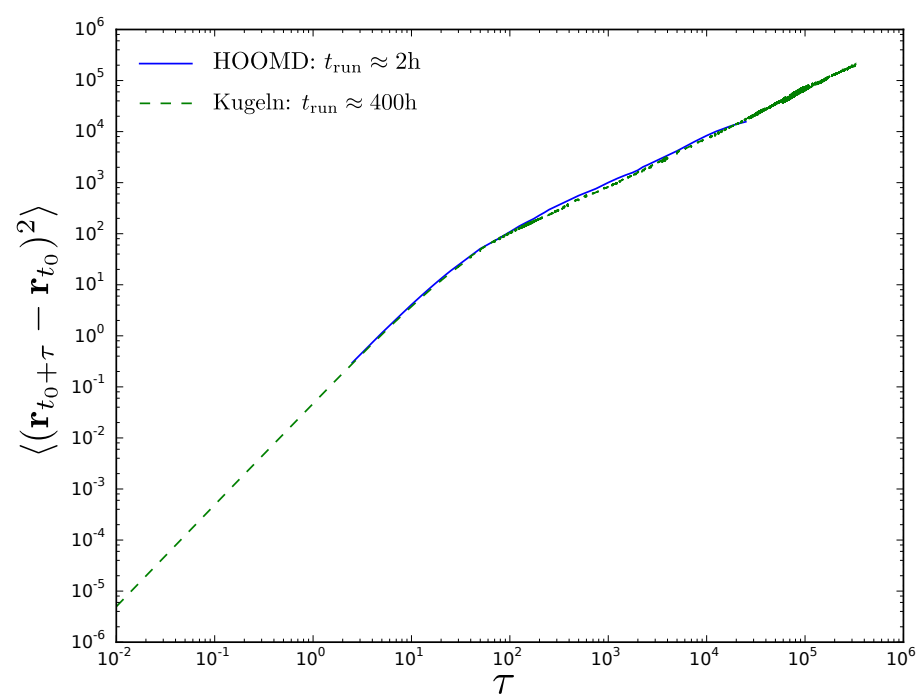

Figure 2.3: A comparison of the results produced by our own program, "Kugeln", and HOOMD. As we can see in this figure a proper choice of corresponding parameters can generate equivalent systems, while HOOMD is much faster specially in dense systems due to its parallel computing.

\subsubsection{Implementation of obstacles}

We implement the heterogeneity by means of immobile spherical obstacles of radius $R_{0}$. Particles experience the same interaction with obstacles as with the other particles. In chapter 5 an active Lorentz model is discussed thoroughly in the first section. The obstacles in this section are initially inserted randomly in the system with the only constraint that they do not overlap. Different scenarios for the geometry of the obstructions leads to various collective behavior of active particles and our goal in the next chapters is to try to shed some light on this issue.

\subsubsection{Measurements}

Before proceeding to studying the dynamics of active particles in different heterogeneous media, it is instructive to compare some predictions of our theory model to the simulation results in dilute systems (where collisions that are ignored in the analytical approach are rare). The results presented below, also develop our intuition about the significance of different model parameters. 


\subsubsection{Velocity along the initial orientation}

Starting from Eq. 2.22 our analytical calculations suggest that in the steady state $(t \rightarrow \infty)$,

$$
\langle\mathbf{v}(t+\tau) \cdot \mathbf{n}(t)\rangle=\sqrt{x}\left[\frac{e^{-\frac{y}{2} \tau}}{c-\frac{y}{2}}-\frac{y}{c^{2}-\frac{y^{2}}{4}} e^{-c \tau}\right] .
$$

Hence, we can write the quantity plotted in Fig. 2.4 as

$$
\operatorname{VCF}(\tau)=\frac{\langle\mathbf{v}(t+\tau) \cdot \mathbf{n}(t)\rangle}{\langle\mathbf{v}(t) \cdot \mathbf{n}(t)\rangle}=\frac{\left(c+\frac{y}{2}\right) e^{-\frac{y}{2} \tau}-y e^{-c \tau}}{c-\frac{y}{2}} .
$$

The averaging in numerical analysis is over the particles as well as time. In other words,

$$
\langle\mathbf{v}(t+\tau) \cdot \mathbf{n}(t)\rangle=\frac{1}{T} \sum_{t=t_{1}, t_{2}, \ldots}^{t_{T}} \frac{1}{N_{p}} \sum_{i=1}^{N_{p}} \mathbf{v}_{i}(t+\tau) \cdot \mathbf{n}_{i}(t) .
$$

$\operatorname{VCF}(\tau)$ measures the average alignment of particle's orientation with its velocity at a later time and hence vanishes at large times, when the velocity completely loses its correlation with the previous orientation. At small times however, as long as the orientation has not changed much, driving leads to the increase of velocity along $\mathbf{n}(t)$. The time when the slope of this quantity changes $\operatorname{sign}\left(\left.\frac{\mathrm{d}}{\mathrm{d} \tau} \operatorname{VCF}(\tau)\right|_{\tau_{0}}=0\right)$ can be exactly calculated:

$$
\tau_{0}=\frac{1}{\frac{y}{2}-c} \ln \left(\frac{y / 2+c}{2 c}\right),
$$

as well as the height of the corresponding maximum in $\operatorname{VCF}(\tau)$ :

$$
\operatorname{VCF}\left(\tau_{0}\right)=2 e^{-c \tau_{0}}=2\left(\frac{c+y / 2}{2 c}\right)^{\frac{c}{c-\frac{y}{2}}}
$$

Equations 2.32, 2.33 confirm that the peaks in this quantity occur at smaller times and with larger height as $y$ increases, as one can see in Fig 2.4. Differences between the theoretical (dashed lines) and simulation arguments in this figure are due to collisions and have been seen to vanish in very dilute systems.

We have defined activity as the parameter that measures the alignment of velocity and orientation of a particle (Eq. 2.27) and have predicted it to decrease with $y$, the rotational diffusion constant (Eq. 2.23). Fig. 2.4 confirms that such a definition leads to the emergence of persistence of the motion in active particle, which is a key aspect in such systems.

\subsubsection{Displacement along the initial orientation}

An answer to the question of how long particles "remember" their initial orientation can be delivered by investigating $\langle\mathbf{v}(t+\tau) \cdot \mathbf{n}(t)\rangle$, given 


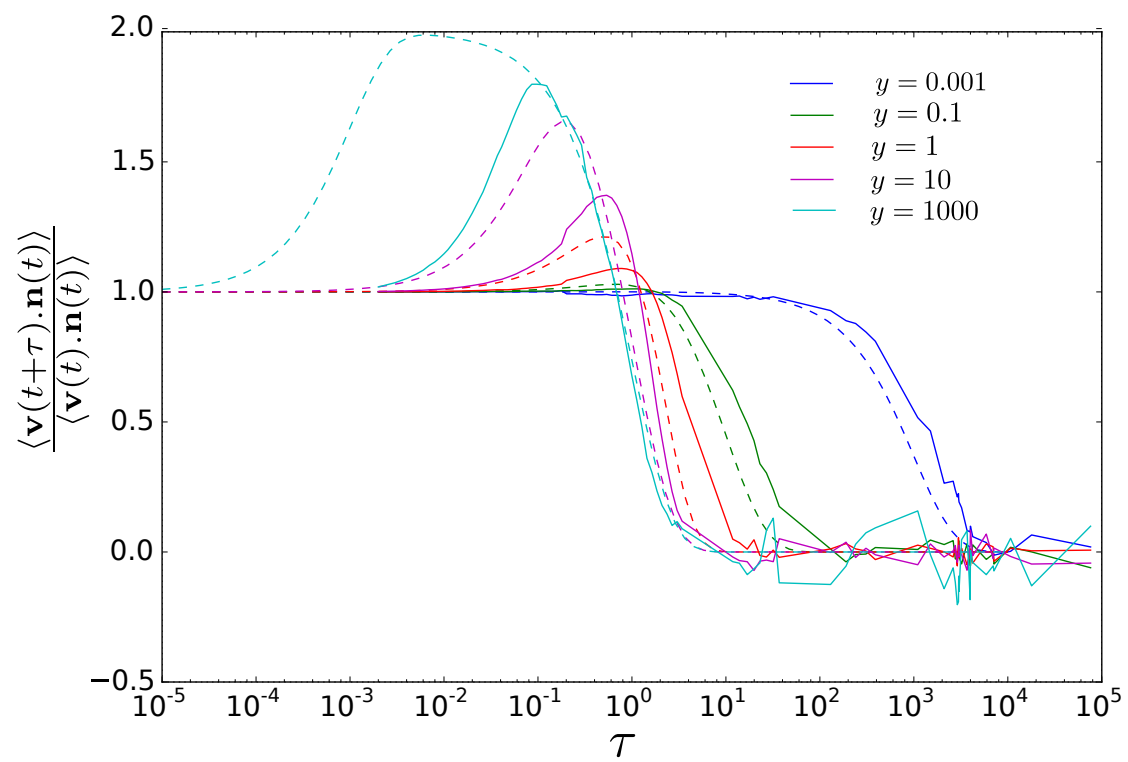

Figure 2.4: Comparison of theory with simulation results for different values of $y$. The packing fraction is 0.01 to reduce the rate of collisions and hence have a better agreement between theory and simulations.

by the previous part. To answer how far a particle can travel before it deflects from its initial orientation, we need to look at $\left\langle\Delta r^{\|}(\tau)\right\rangle=$ $\langle(\mathbf{r}(t+\tau)-\mathbf{r}(t)) \cdot \mathbf{n}(t)\rangle$. This quantity can be analytically derived by integrating eq. 2.29:

$\left\langle\Delta \mathrm{r}^{\|}(\tau)\right\rangle=\sqrt{x}\left[-\frac{2}{(\mathrm{c}-\mathrm{y} / 2) \mathrm{y}} e^{-y \tau / 2}+\frac{\mathrm{y}}{\mathrm{c}\left(\mathrm{c}^{2}-\mathrm{y}^{2} / 4\right)} e^{-\mathrm{c} \tau}+\frac{2 \mathrm{c}(\mathrm{c}+\mathrm{y} / 2)-\mathrm{y}^{2}}{\mathrm{cy}\left(\mathrm{c}^{2}-\mathrm{y}^{2} / 4\right)}\right]$,

which reveals that $\left\langle\Delta \mathrm{r}^{\|}(\tau)\right\rangle$ grows linearly for small $\tau$ and saturates at large times. The saturating begins when particles' displacements lose correlation with their initial orientations, namely when the first term on the RHS of the equation below vanishes:

$$
\left\langle\Delta \mathrm{r}^{\|}(\tau+2 \Delta \tau)\right\rangle=\langle(\mathbf{r}(\tau+2 \Delta \tau)-\mathbf{r}(\tau+\Delta \tau)) \cdot \mathbf{n}(0)\rangle+\left\langle\Delta \mathrm{r}^{\|}(\tau+\Delta \tau)\right\rangle,
$$

Saturation is expected to occur at around the time when $\langle\mathbf{v}(t+\tau) \cdot \mathbf{n}(t)\rangle$ drops to a small value. One can define the "correlation time", $\tau_{\text {corr }}$, as the time when $\langle\mathbf{v}(t+\tau) . \mathbf{n}(t)\rangle$ drops to half of its initial value. From Fig. $2.4, \tau_{\text {corr }} \sim 1500,15,3,2,1.5$ for $y=0.001$ to 1000 respectively. These values compare relatively well with the beginning of the saturation in Fig. 2.5

The saturation value predicted by Eq. 2.34,

$$
\left\langle\Delta \mathrm{r}^{\|}(\infty)\right\rangle \sim \frac{\sqrt{x}}{\mathrm{y}}
$$

puts emphasis on the fact that the more active, the longer particles move along their initial orientation. 


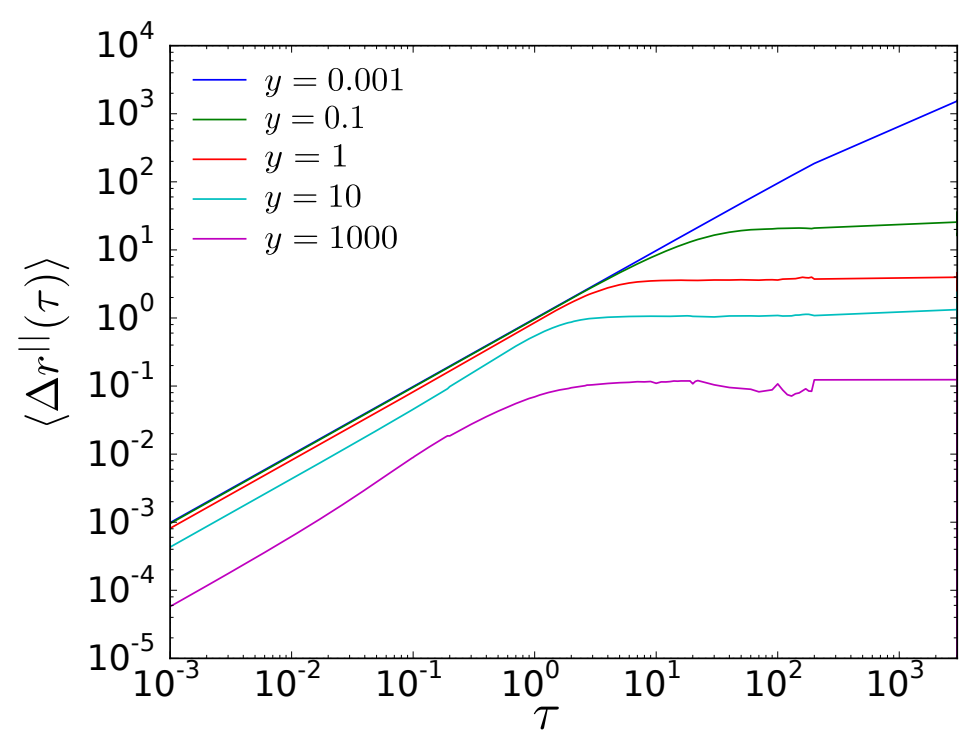

Figure 2.5: Mean displacement along the initial orientation. This quantity converges to a constant value as the displacement in future times loses correlation with the initial orientation.

\subsubsection{Mean square displacement}

The mean square displacement (MSD) of particles in systems with different magnitude of activity is plotted in Fig. 2.6. Activity increases the translational diffusion coefficient as expected from Eq. 2.24. More specifically, since we adapt $x$ to the increase of $y$ (explained in the last paragraph before Eq. 2.28) the diffusion coefficient in our simulations is expected to be

$$
D_{t}=\frac{2 E_{t}}{c} \frac{c+y / 2}{y}
$$

where $E_{t}$ is the translational kinetic energy of the system which we keep constant throughout all simulations. Hence $D_{t}$ diverges as activity is increased $(y \rightarrow 0)$ and saturates to a value specified by $E_{t}$ in the passive limit. Fig. 2.7 compares the result of simulations with the analytical estimation for $D_{t}$. 


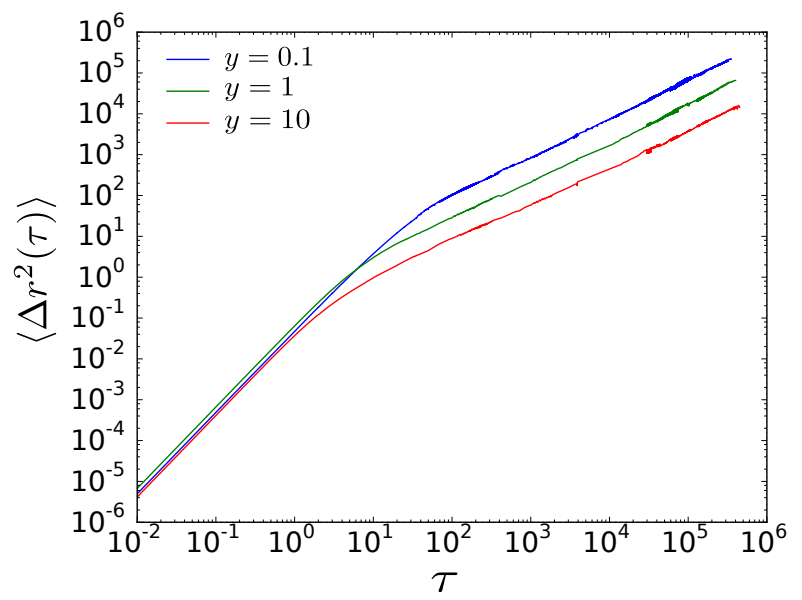

Figure 2.6: MSD for packing fraction $\phi=0.01$ and different activities. The more active a system is, the longer the initial ballistic regime lasts and the larger the translational diffusion coefficient becomes.

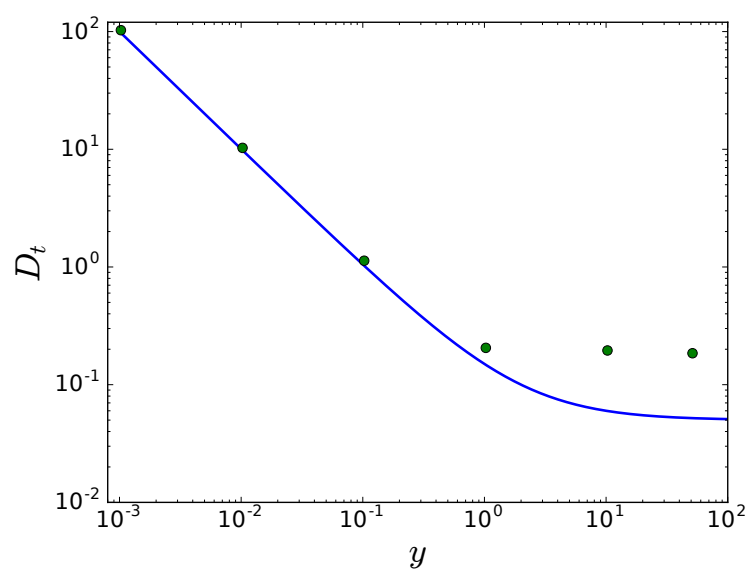

Figure 2.7: Translational diffusion coefficient in systems with $E_{t}=0.05$, and different $y$. The solid line represents our theoretical prediction for $D_{t}$ (Eq. 2.37) and the bullets represent our simulation results. 
In real environments, active particles move in the presence of walls and soft or solid obstacles. The examples range from microorganisms in confined physiological environments, to larger swimmers in the ocean. A wide range of experiments reveal the tendency of microswimmers to traverse along the boundaries of an obstacle for a prolonged time [52, 90-92]. Bacteria for example have been observed to be trapped at surfaces [48], swim in circles [13] or experience suppressed tumbling near them [93]. Such long "residence times" near walls and obstacles have been recognized to build the early stages of the formation of complex aggregates such as biofilms [94-96], hence studying them facilitates the design of systems where the formation of such aggregates can be controlled. Several mechanisms have been proposed to explain the long residence times and therefore accumulation of active particles at surfaces, among them hydrodynamic interactions was initially suggested to play the central role. However, it is known now that the particle-obstacle dynamics results from an interplay of particle's shape, noise and direct collisions [97]. It has been particularly shown that the combination of Brownian motion and selfpropulsion can already give rise to the observed accumulation, in the absence of any explicit hydrodynamic interaction between particles and the wall [51].

In this chapter we investigate the interaction of a single particle with a hard planar wall and then spherical immobile obstacles. Active particles in our model tend to experience successive collisions with the wall after they first hit it, consequently one can define a residence time during which the particle moves close and along the surface. Here we study some general aspects of the residence time.

\section{I RESIDENCE ON A FLAT WALL}

An important distinction between active and passive particles is their orientational persistence. Particles keep pushing in the same direction for some time comparable to the inverse of the rotational diffusion coefficient. It is evident from eq. 2.23 that in our model this time decreases as $y$ is increased, e.g. as the activity is reduced. In the case of collisions with an infinite planar wall, the self-propelling force returns an active particle to the wall after it bounced back in the first contact. This procedure repeats and the tangent component of particle's velocity to the wall, slides it on the wall until its orientation points away from the wall. Hence we define the residence time, $\tau_{\text {res, }}$ 
as the time from the first contact with the wall until the orientation is for the first time parallel to the wall.

All we need to know about the residence times, can be extracted from its distribution, $P\left(\tau_{\text {res }}\right)$, and hence we try to find this function either analytically or computationaly below. We expect $P\left(\tau_{\text {res }}\right)$ to depend on particle's orientation when it first collides the wall and its rotational diffusion. Consider the two following cases: a particle striking the wall perpendicularly and a particle that hits the wall almost parallel to it. The particle in the former case is expected to wander along the wall for a longer time, as compared to the latter which only briefly touches the wall. Similarly a particle with smaller rotational diffusion is expected to linger at the wall for a longer time in average, as compared to a particle that requires smaller time to reorient and leave the wall. The dependence of the residence time on other parameters is less intuitive and will be discussed below.

The residence time on the wall can possibly be calculated through the approach of first passage time problems. In principle $\tau_{\text {res }}$ in our model can be considered as the first passage time of the angle $\theta(t)=$ $\theta_{i}+\int_{0}^{t} \omega\left(t^{\prime}\right) d t^{\prime}$ from the incident angle $\theta_{i}$ (namely the angle between particle's orientation and normal to the wall when it first hits the wall, demonstrated in Fig. 3.1) at time 0 to $\pm \pi / 2$ at time $t$, where $\omega$ is the Ornstein-Uhlenbeck process defined by Eq. 2.9b. The distribution of the first passage time for the Ornstein-Uhlenbeck process has been a research topic of its own [98-100].

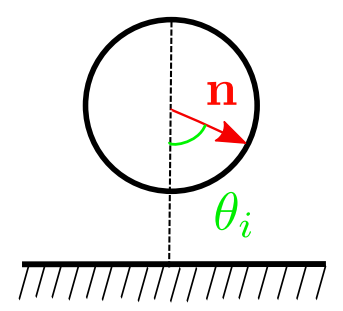

Figure 3.1: Demonstration of the incident angle, $\theta_{i}$, in collisions with a wall.

In [101], the related problem of residence times of active microswimmers against a wall was solved, neglecting inertial terms. One of the results of that paper is that the distribution depends on the incident angle , $\theta_{i}$, and shows a peak at a finite typical residence time which shifts towards smaller values for larger $\theta_{i}$. The average residence time in ref. [101] is analytically derived and found to decrease with particle's rotational diffusivity and $\theta_{i}$. Similar observations are qualitatively found by our simulations (see Fig. 3.2) and will be thoroughly discussed below. However a quantitative comparison of our simulation results to this work is not viable since the inertial terms are known to make a difference [102]. The inertial term in the equation for rotational motion changes the order of the differential equation 
and introduces an extra dependence of $\tau_{\text {res }}$ on the initial angular velocity, $\omega_{i}$.

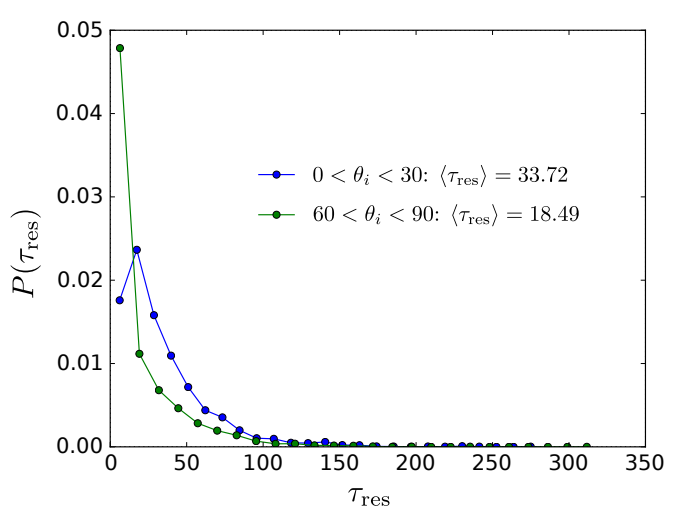

Figure 3.2: Distribution of the residence time of a particle on a wall, given different ranges of the incident angle. The measurement is over roughly 1000 residence events in 1000 systems with $y=0.1$.

In order to compute $\tau_{\text {res, }}$ I run several simulations for a single particle with wall boundary. The system size is chosen very large compared to the particle radius in order to represent an infinite wall. I consider a fixed time window in my simulation time during which the particle in all my systems experiences in average 1000 "residence events". I define residence event as the succession of collisions with the wall within a residence time. I compute $\tau_{\text {res }}$ for such events, together with $\theta_{i}$, and $\omega_{i}$.

Plotting the distribution of $\tau_{\text {res }}$ for all the possible incident angles (Fig. 3.3) and a given range of $\omega_{i}$, reveals that there is a very small probability for the occurrence of very large $\tau_{\text {res. }}$. We are going to ignore those irrelevant residence times in the following.

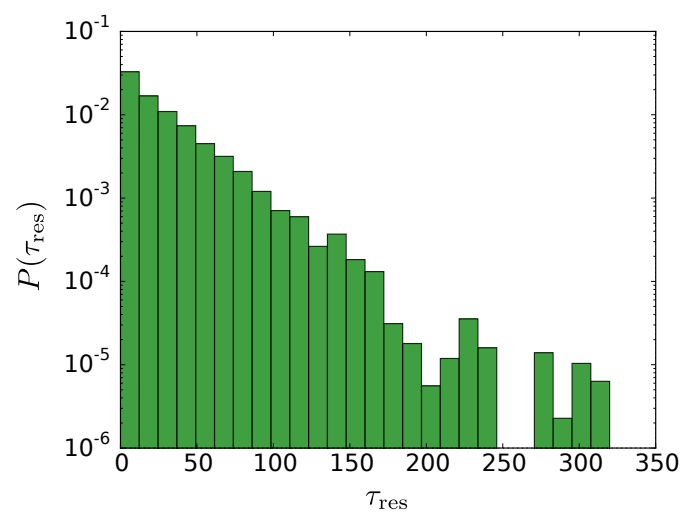

Figure 3.3: Distribution of the residence time of a particle with $y=0.1$ and $\left|\omega_{i}\right|<0.2$ on a wall. 
Fig. 3.4 illustrates $\mathrm{P}\left(\tau_{\text {res }}\right)$ for different number of simulation runs. The statistics for 100 simulation runs seems to be good enough and we may stick to that for the rest of our analysis in this section.

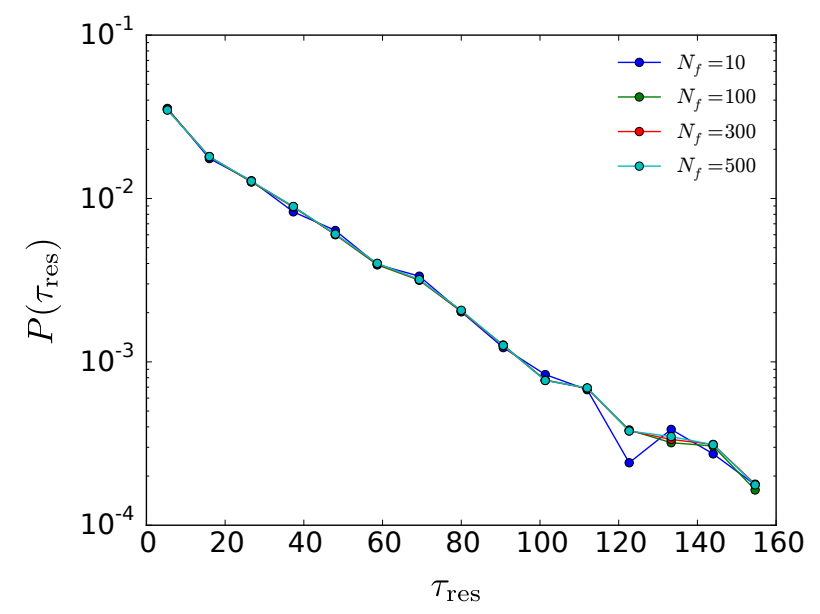

Figure 3.4: Distribution of the residence time of a particle with $y=0.1$ and $\left|\omega_{i}\right|<0.2$ on a wall, measured for $N_{f}$ systems.

The residence time is expected to depend on $\omega_{i}$. Fig. 3.5 demonstrates $\mathrm{P}\left(\tau_{\text {res }}\right)$ for different ranges of $\omega_{i}$ : we observe a faster decay of the mean residence time by the increase of $\omega_{i}$. Rapid change of the orientation from almost normal incidents to $\theta(t)= \pm \pi / 2$ due to a large $\omega_{i}$, can explain this observation.

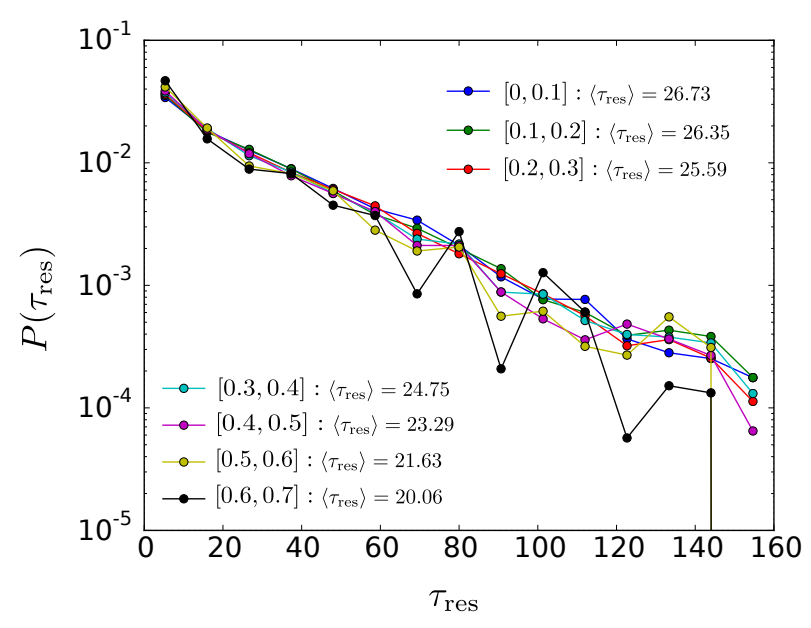

Figure 3.5: Distribution of the residence time of a particle with $y=0.1$ on a wall for different ranges of $\left|\omega_{i}\right|$. Note that according to Eq. 2.25, the average magnitude of $\omega_{i}$ is about 0.224 when $y=0.1$. This explains why we have poor statistics as $\omega_{i}>0.5$. 
Dependence of $\mathrm{P}\left(\tau_{\text {res }}\right)$ on the incident angle is illustrated in Fig. 3.6. Given the discussion above, it is no surprise that $\left\langle\tau_{\text {res }}\right\rangle$ decreases as $\theta_{i}$ approaches $\pi / 2$ (Fig. 3.7). The dashed line in Fig. 3.7 is the estimation for $\left\langle\tau_{\text {res }}\right\rangle$ in the overdamped model of Schaar et al.[101] for similar parameters as our model.

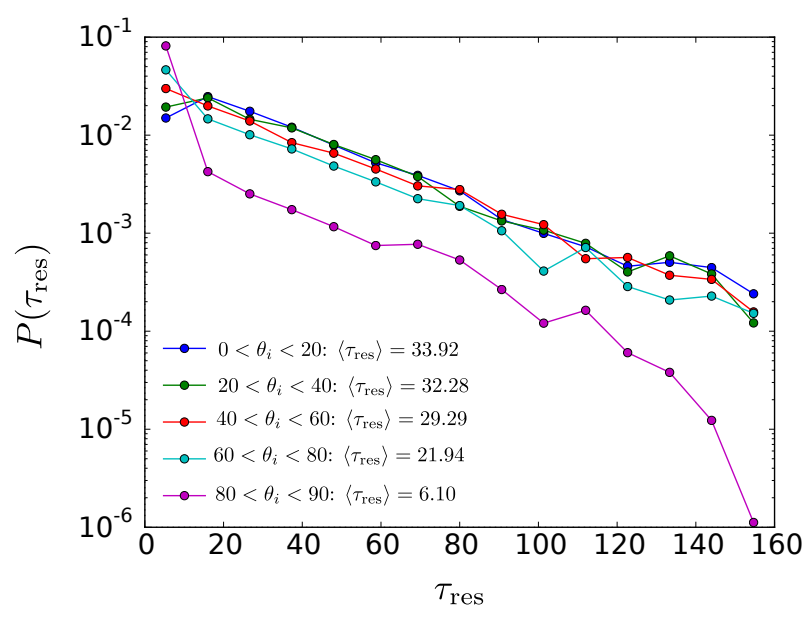

Figure 3.6: Distribution of the residence time of a particle with $y=0.1$ on a wall for different ranges of the incident angle $\theta_{i}$. The long exponential tail of the distribution shifts vertically downwards as the angle between the incident orientation and the normal to the wall increases.

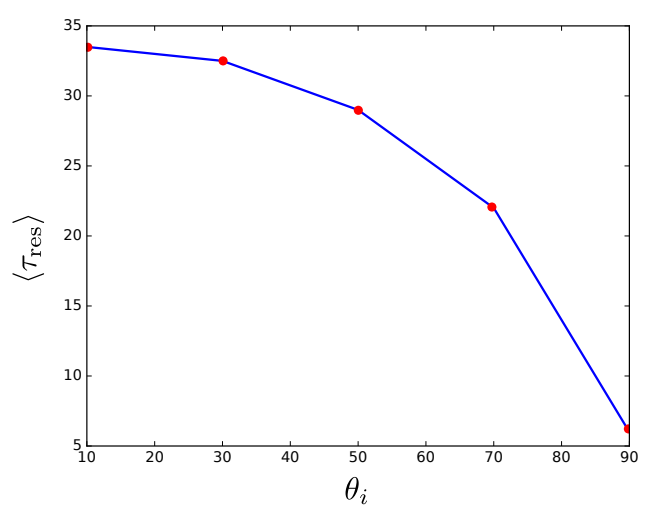

Figure 3.7: Average of the residence time on a flat wall, given the incident angle $\theta_{i}$. The average is over 500 systems with $y=0.1$.

The above plots are all only for a given $\omega_{i}$ or a given $\theta_{i}$ and provide us with e.g. no information about the dependence of $\left\langle\tau_{\text {res }}\right\rangle$ on $\omega_{i}$ at a specific $\theta_{i}$. We need to plot our quantities of interest versus both $\omega_{i}$ and $\theta_{i}$. Fig. 3.8 shows $\left\langle\tau_{\text {res }}\right\rangle$ versus $\omega_{i}$ and $\theta_{i}$ in an active and passive system. The value of $\left\langle\tau_{\text {res }}\right\rangle$ is quite different in these two systems (the range of the investigated $\omega_{i}$ is different in passive and active systems as explained above). One can also infer from this figure that $\left\langle\tau_{\text {res }}\right\rangle$ 
does not appear to change strongly with $\omega_{i}$.
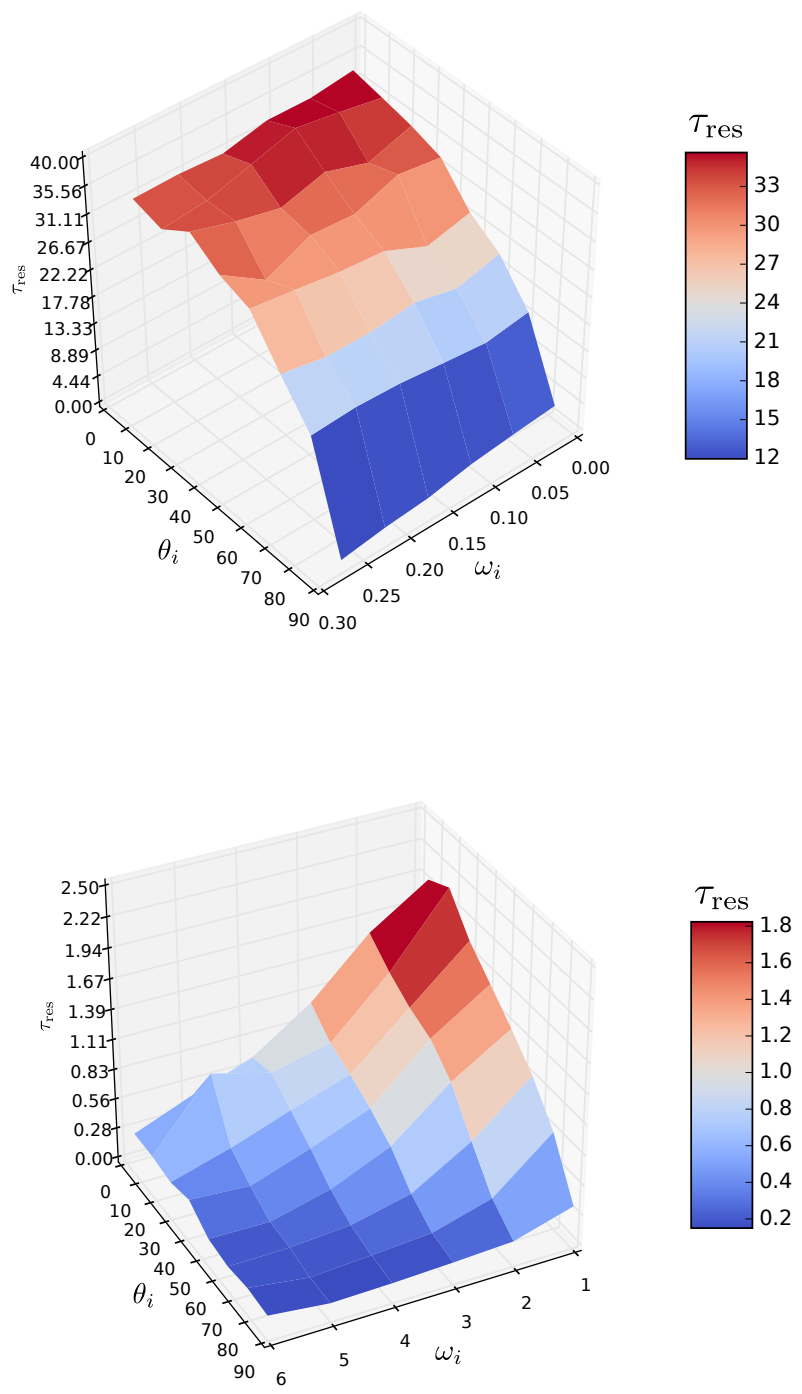

Figure 3.8: $\left\langle\tau_{\text {res }}\right\rangle$ as a function of $\theta_{i}$ and $\omega_{i}$ for $y=0.1$ (top) and $y=10$ (bottom). In active systems, this quantity changes only subtly with the initial angular velocity, but decreases considerably as the incident angle approaches $90^{\circ}$.

Other than the initial angular velocity and orientation, the dependence of the residence time on our model parameters, $x$ and $y$ needs to be investigated as well. For a given $y$ one expects $\tau_{\text {res }}$ on an infinite wall to be indifferent to the particle's speed, i.e. $x$. This is confirmed by Fig. 3.9. Activity on the other hand has a strong effect on $\tau_{\text {res }}$ : in the limit of zero $y$ particles stay close to the wall for an infinitely long time (Fig. 3.10). 


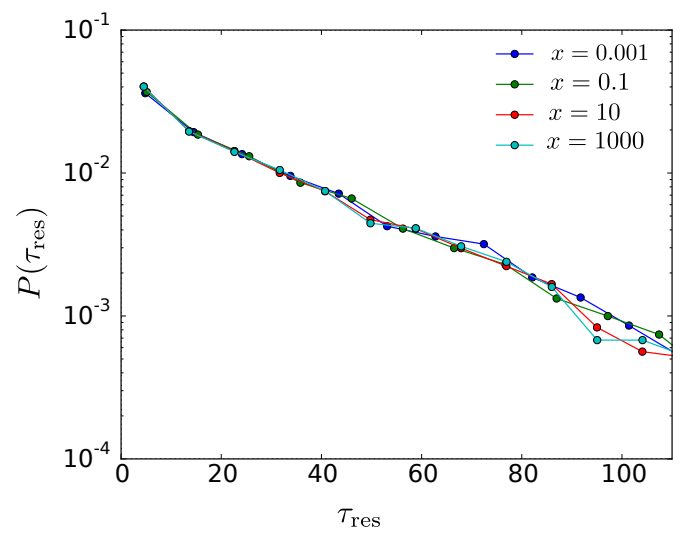

Figure 3.9: Distribution of the residence time of a particle with $y=0.1$ and different $x$ on a wall. The residence times on a planar wall are independent of $x$ and determined by the dynamics of the angle only.

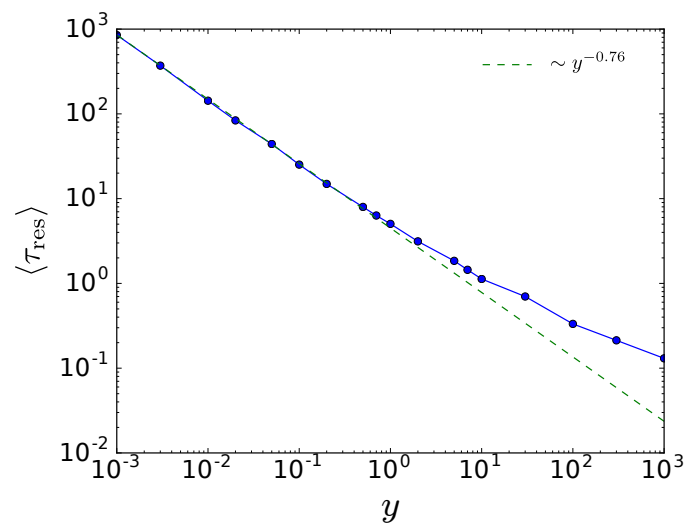

Figure 3.10: The average residence time on a wall as a function of $y$. This quantity diverges at zero $y$ (high activity) in the form of a power-law.

$\mathrm{P}\left(\tau_{\text {res }}\right)$ drops as $\exp \left(-\tau_{\text {res }} / \tau_{0}\right)$ at intermediate to long times with a characteristic time, $\tau_{0}$, that is a function of $y$. We have fitted an exponential to $P\left(\tau_{\text {res }}\right)$ for different $y$ and plotted $\tau_{0}$ as a function of $y$ (Fig. 3.11).

Furthermore we compute the distance the particle moves along a wall (in other words the distance between the two furthest points on the wall, touched by the particle) during one residence event, $d_{\text {res }}$. The distribution of this quantity is shown in Fig. 3.12 for two different $\theta_{i}$. The average of $d_{\text {res }}$ in an active system is larger than in a passive one and its overall dependence on $\theta_{i}$ and $\omega_{i}$ is roughly similar to $\tau_{\text {res }}$ (Fig 3.13). $\left\langle d_{\text {res }}\right\rangle$ can roughly be estimated as the mean displacement of the particle during a residence event and is then expected to have a value close to $\sqrt{2 D_{t} \tau_{\text {res }}}$. Calculating $D_{t}$ for the system in Fig. 3.13 and extracting $\left\langle\tau_{\text {res }}\right\rangle$ for small angles from Fig. 3.8, the estimation 


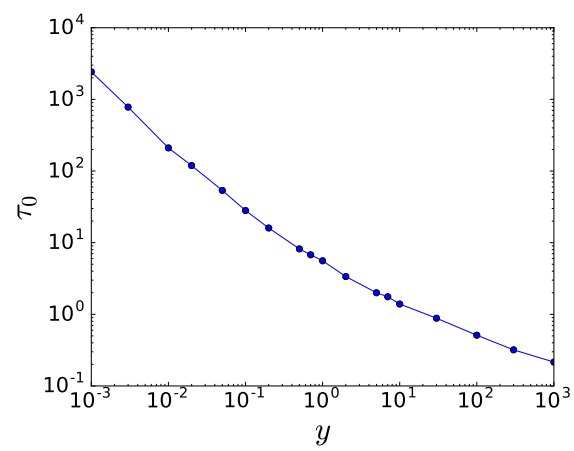

Figure 3.11: The characteristic time scale $\tau_{0}$, computed by fitting an exponential $\exp \left(-\tau_{\text {res }} / \tau_{0}\right)$ to $P\left(\tau_{\text {res }}\right)$, as a function of $y$.

$\left(\left\langle\mathrm{d}_{\text {res }}\right\rangle \approx \sqrt{2 \mathrm{D}_{\mathrm{t}} \tau_{\text {res }}} \simeq 6\right)$ is found to be similar to the value observed in Fig. 3.12 at small angles: $\left\langle d_{\text {res }}\right\rangle \simeq 4$. The agreement is of course not expected to be exact as $\tau_{\text {res }}$ may not be long enough to assume the motion is diffusive.

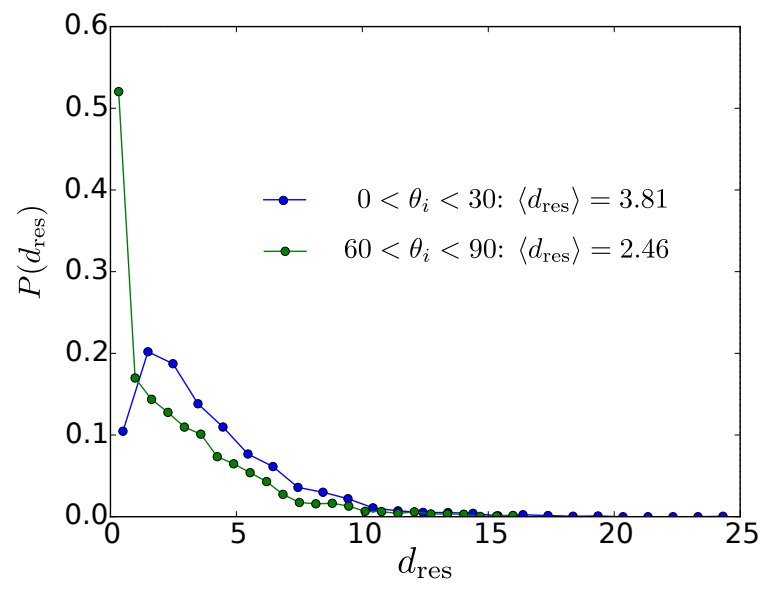

Figure 3.12: Distribution of the distance between two furthest points touched by an active particle on the wall during a residence event, for two different ranges of incident angle.

\subsection{INTERACTION WITH OBSTACLES}

Active particles in real environments interact not only with planar walls, but also with obstacles of different geometries. Immersed passive colloids in a fluid, large aggregate of dead cells, biological polymers are some examples of real obstacles an active particle may interact with. Convex obstacles have been realized in several experiments $[52,92,103]$ and shown to trap active particles when their size is sufficiently larger than the particle. The capture of active particles by such obstacles is often associated with hydrodynamic interac- 

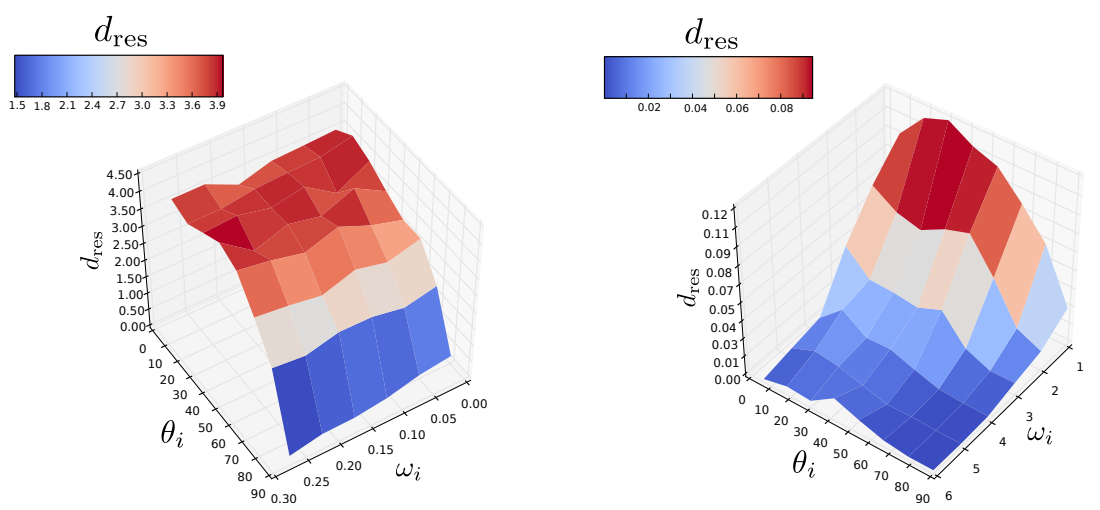

Figure 3.13: $\left\langle\mathrm{d}_{\text {res }}\right\rangle$ as a function of $\theta_{i}$ and $\omega_{i}$ for $y=0.1$ (left) and $y=$ 10 (right). In active systems this quantity changes only subtly by the initial angular velocity, but decreases considerably by enlarging the incident angle, similar to the average residence time (Fig. 3.8).

tions $[52,53,92]$, but steric forces are also known to play a major role for some types of active particles [103].

In this section we study the motion of a single particle in the vicinity of a stationary spherical obstacle. Analytical calculation of $p\left(\tau_{\text {res }}\right)$ and $\left\langle\tau_{\text {res }}\right\rangle$ is even more demanding than the case of a wall due to the non-zero curvature and a more complex boundary condition. We compare the result of our simulations for the residence time to the case of a flat wall and learn how this time is influenced by our model parameters.

Fig. 3.14 reveals that the residence time on an obstacle 1o times larger than the particle still has an exponential distribution but its average is smaller than in the case of a wall. Obstacles with $R_{o} / R_{p}=30$ or larger, reproduce almost the same results as a flat wall. One can make sense of these results by considering a particle with normal incident that moves along an arc of magnitude $\Delta \theta$ around an obstacle before its orientation leads it away from it. The distance that this particle travels on the obstacle is expected to be comparable to the value of $\left\langle d_{\text {res }}\right\rangle$ found for a flat wall; in other word $\left(R_{o}+R_{p}\right) \Delta \theta=\left\langle d_{\text {res }}\right\rangle$. On the other hand, the particle's change of orientation, $\pi / 2-\Delta \theta$, is expected to satisfy $\left\langle(\pi / 2-\Delta \theta(t))^{2}\right\rangle=y t$, and the time of the traveling on the obstacle is expected to be comparable with the residence time on a flat wall: $t=\left\langle\tau_{\text {res }}\right\rangle$. We conclude that the threshold obstacle radius, $R_{o}^{*}$, which reproduces the same results as the wall can be roughly estimated as

$$
R_{o}^{*}+R_{p}=\frac{\left\langle d_{\text {res }}\right\rangle}{\mid \pi / 2-\sqrt{y\left\langle\tau_{\text {res }}\right\rangle}},
$$

which gives $R_{0}^{*} \approx 30$ for $x=y=0.1$ and the values for $\left\langle\tau_{\text {res }}\right\rangle$ and $\left\langle\mathrm{d}_{\text {res }}\right\rangle$ that our simulation results suggest $\left(\left\langle\tau_{\text {res }}\right\rangle_{\theta_{i}=0} \approx 29\right.$ and 
$\left\langle\mathrm{d}_{\text {res }}\right\rangle_{\theta_{i}=0} \approx 4$ ). From here on, we keep $R_{\mathrm{o}} / R_{p}=10$ and study the

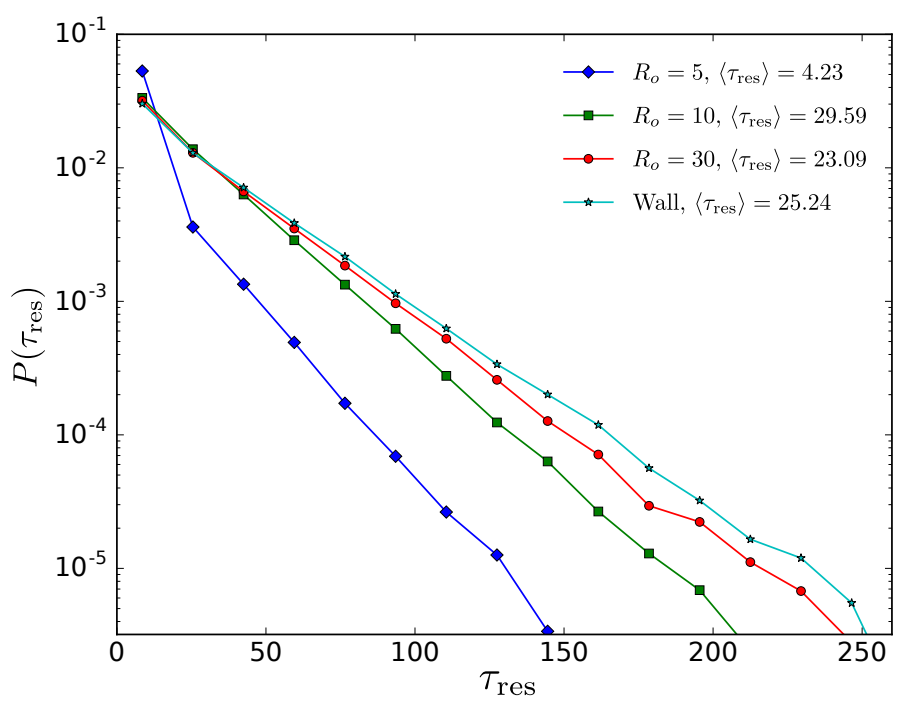

Figure 3.14: Comparison of the distribution of the residence time on a wall and on disk-like obstacles of different radii in systems with $y=0.1$.

dependence of the distribution and average of the residence time on $x, y$, and the incident angle $\theta_{i}$.

Fig. 3.15 and 3.16 illustrate the distribution of residence times of an active particle with different $y$ and different $x$ on an obstacle.

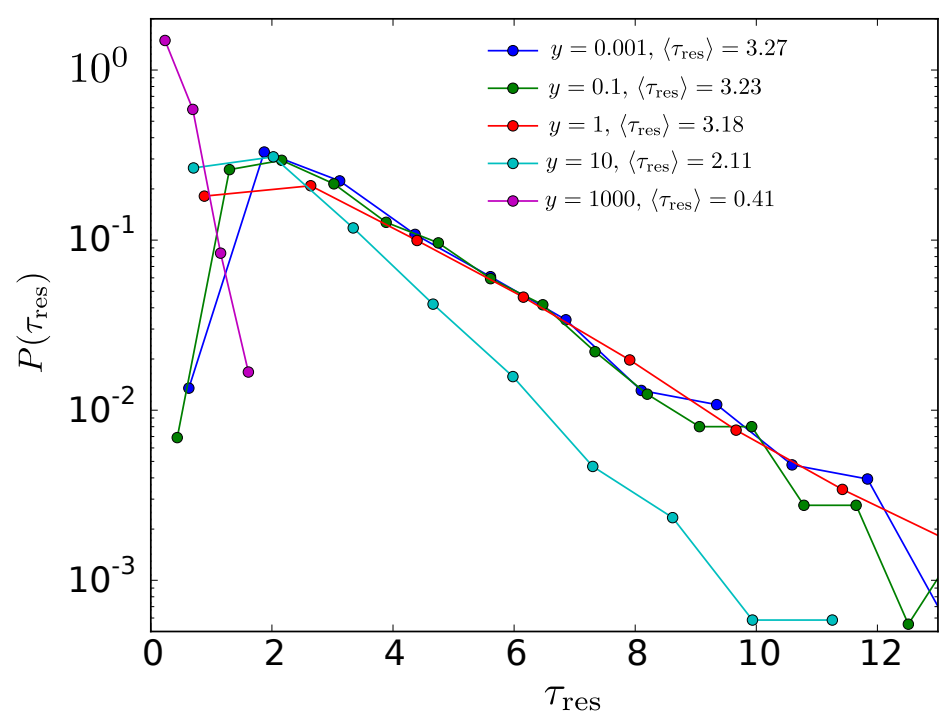

Figure 3.15: Distribution of the residence time on an obstacle with radius $R_{\mathrm{o}} / R_{p}=10$ in systems with different $y$. Number of bins vary between 20 to 5 for $y=0.001$ to $y=1000$, and there are in average 400 points in each bin. 


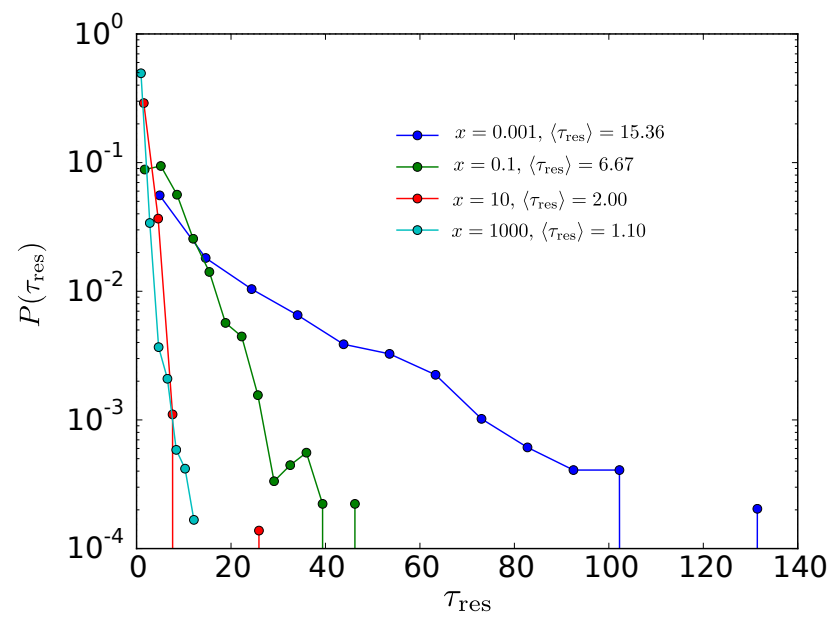

Figure 3.16: Distribution of the residence time on an obstacle with radius $R_{\mathrm{o}} / R_{\mathrm{p}}=10$ in systems with different $x$. The distribution changes with $x$, unlike the case of a flat wall.

According to these results, the average residence time grows as $y$ decreases, e.g. as particle's activity increases. This is in fact what we have learned from the wall case. However unlike residence on a wall, the distribution of $\tau_{\text {res }}$ here converges to a certain function (and its mean to a certain value) as $y \rightarrow 0$. The limited size of an obstacle prohibits infinitely long residence times.

Furthermore, unlike the wall case, increasing $x$ (and hence the speed) decreases the residence time on an obstacle, because it increases the distance particles bounce off the obstacle and therefore decreases the number of successive collisions with the obstacle. As a result the particle with a larger $x$ leaves the obstacle sooner and has a smaller $\tau_{\text {res }}$ and $d_{\text {res. }}$ One can see this in Fig. 3.17.

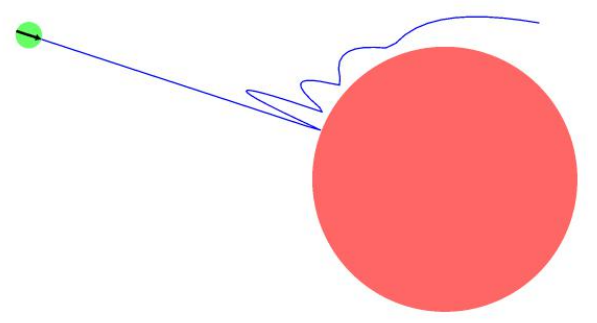

Figure 3.17: Trajectory of an active particle with $x=100$ (left) and $x=0.1$ (right). Speedy particles (associated with large $x$ ) cover longer distances tangent to the obstacle's circumference in bouncing off the surface and hence slip off the obstacle quicker as compared to slower particles. 
The decrease of the number of successive collisions with an obstacle (within a residence event) by an increase of $x$ is shown in Fig. 3.18.

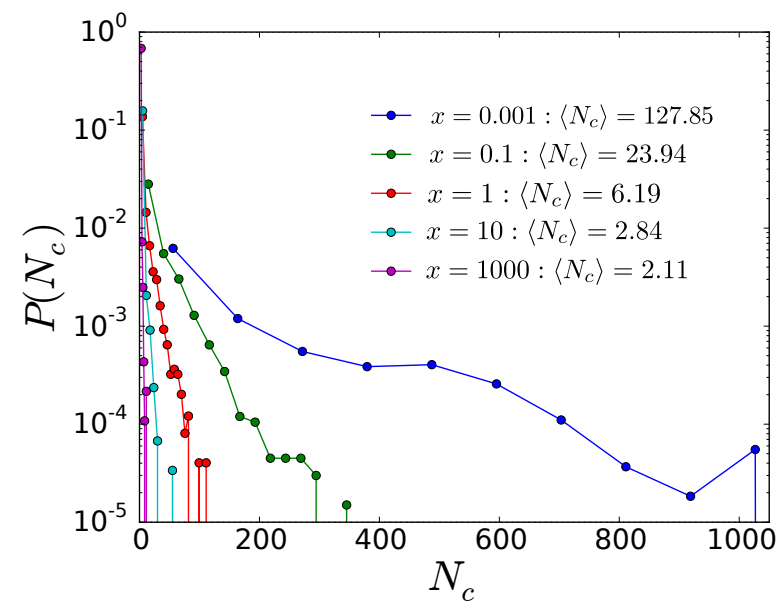

Figure 3.18: Distribution of the number of successive collisions with an obstacle that a particle experiences during its residence on the obstacle. For systems with $y=0.1$ and $R_{o} / R_{p}=10$, the average number of successive collisions decreases dramatically by increasing $x$.

The longest residence times correspond to collisions with a very small initial angle between the orientation and the connecting vector between the obstacle and particle. As one would expect from the case of the wall, the average residence time decreases by increasing this angle $\theta_{i}$ (Fig. 3.19).

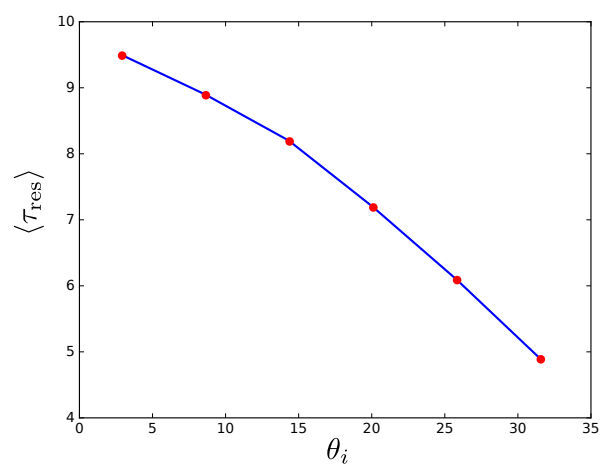

Figure 3.19: Average residence time of particles with $y=0.1$ on an obstacle with $R_{o} / R_{p}=10$ versus different incident angles. Normal incidents correspond to the longest residence times, similar to the case of a flat wall (Fig. 3.7). 
We have learned in the previous chapter that single active particles spend long times in the vicinity of large spherical obstacles and are effectively attracted to them. It is therefore not surprising that increasing the number of particles gives rise to accumulation around the obstacle. In this chapter we study the accumulation and crystallization of particles around large stationary obstacles and later report a peculiar observation: collective rotation of the crystals of particles around the obstacle. We explain the existence and important properties of such rotating crystals through some simple arguments.

\subsection{ACCUMULATION}

Given the attraction of individual active particles to surfaces, it is no surprise that particles accumulate at surfaces and form large clusters. The small residence times of passive particles (Fig. 3.15) provokes no accumulation of such particles around obstacles (see Fig. 4.I).
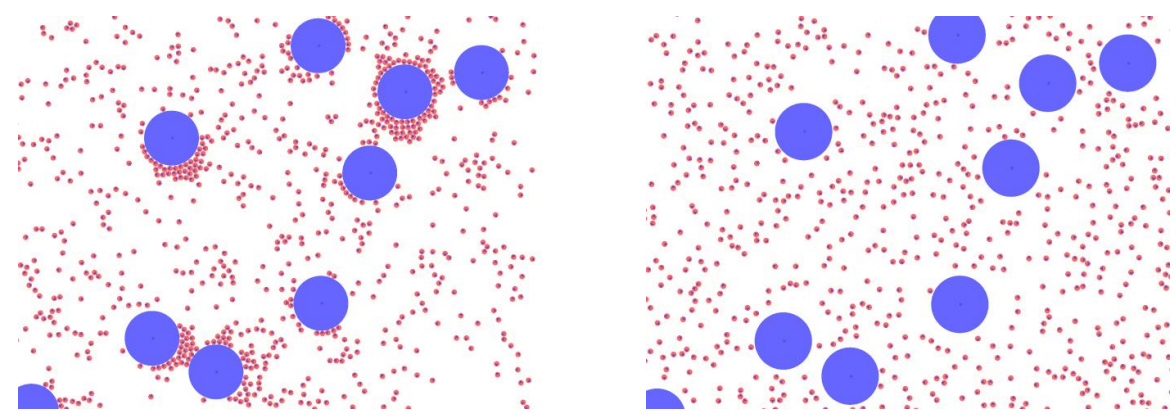

Figure 4.1: Snapshot of the system with $\phi_{p}=0.08, R_{p} / R_{o}=0.1$ and $y=0.001$ (left), $y=10$ (right). Active particles, in contrast to passive ones, form living aggregates around the obstacles.

This accumulation is reflected in the sharp peaks of the pair correlation of the obstacles with the surrounding particles (Figure 4.2), defined as

$g(r)=\frac{L^{2}}{2 \pi r \Delta} \frac{1}{N_{s}} \Sigma_{s} \frac{1}{N_{o}} \Sigma_{i=1}^{N_{o}} \frac{1}{N_{p}} \Sigma_{j=1}^{N_{p}} \theta\left(r+\Delta-\left(r_{i}-r_{j}\right)\right) \theta\left(\left(r_{i}-r_{j}\right)-r\right)$,

where $\theta$ is the Heaviside step function and $\Delta$ is bin size. $\Sigma_{s}$ is the average over $N_{s}$ snapshots, to improve the statistics. $g(r)$ is computed by counting the number of particles within a shell of thickness $\Delta$ at a 
distance $r$ from an obstacle. The prefactor is chosen so that $g(r) \rightarrow 1$ at large $r$. The first peak corresponds to the first layer of particles at distance $R_{p}+R_{o}$ from the center of the obstacle. The next peaks correspond to the outer layers of particles. Figure 4.3 reveals that the height of the first peak increases with activity and the accumulation is absent for passive particles $(y>1)$.

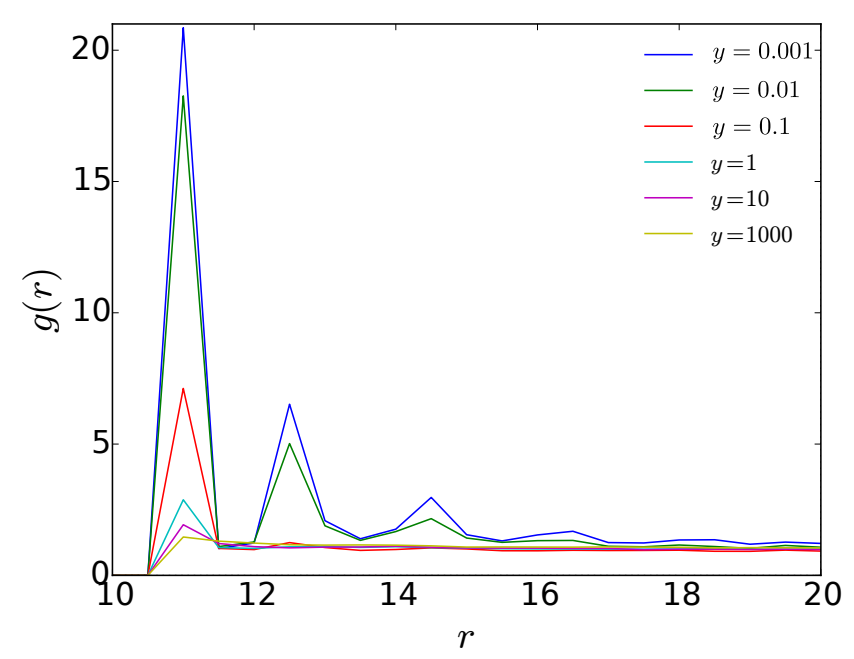

Figure 4.2: Pair correlation function $(\Delta=0.5)$ of systems with $\phi_{\mathrm{o}}=$ $\phi_{p}=0.08$ and different levels of activity. The first, second and third peaks correspond to different layers of particles accumulated around obstacles at distances approximately equal to $R_{o}+R_{p}, R_{o}+3 R_{p}$, and $R_{o}+5 R_{p}$ from the obstacles.

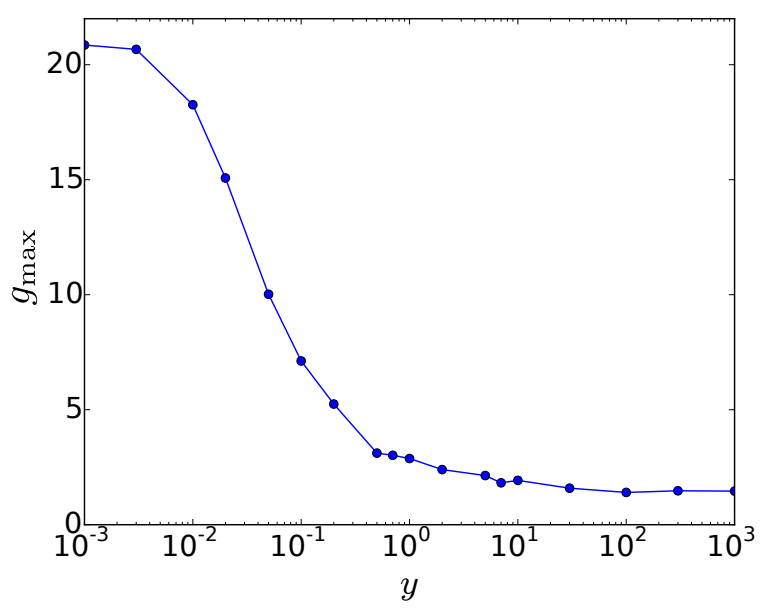

Figure 4.3: Height of the first peak of the pair correlation function versus $y$ for systems in Fig. 4.2. This quantity shows a crossover from the maximum number of particles that can be placed on the circumference of an obstacle in the active regime, to the value expected in a homogeneous fluid in the passive regime. 
The accumulation is further enhanced by increasing the particle density $\phi_{p}=\frac{N_{p} \pi R_{p}^{2}}{L^{2}}$; more layers form around obstacles. Fig. 4.4 illustrates this speculation. Note that the height of the peaks depend on the total number of particles in the system and hence decreases as $\phi_{p}$ increases. What reveals the large accumulation around obstacles is the number of layers around them which is reflected in the number of successive peaks in $\mathrm{g}(\mathrm{r})$.
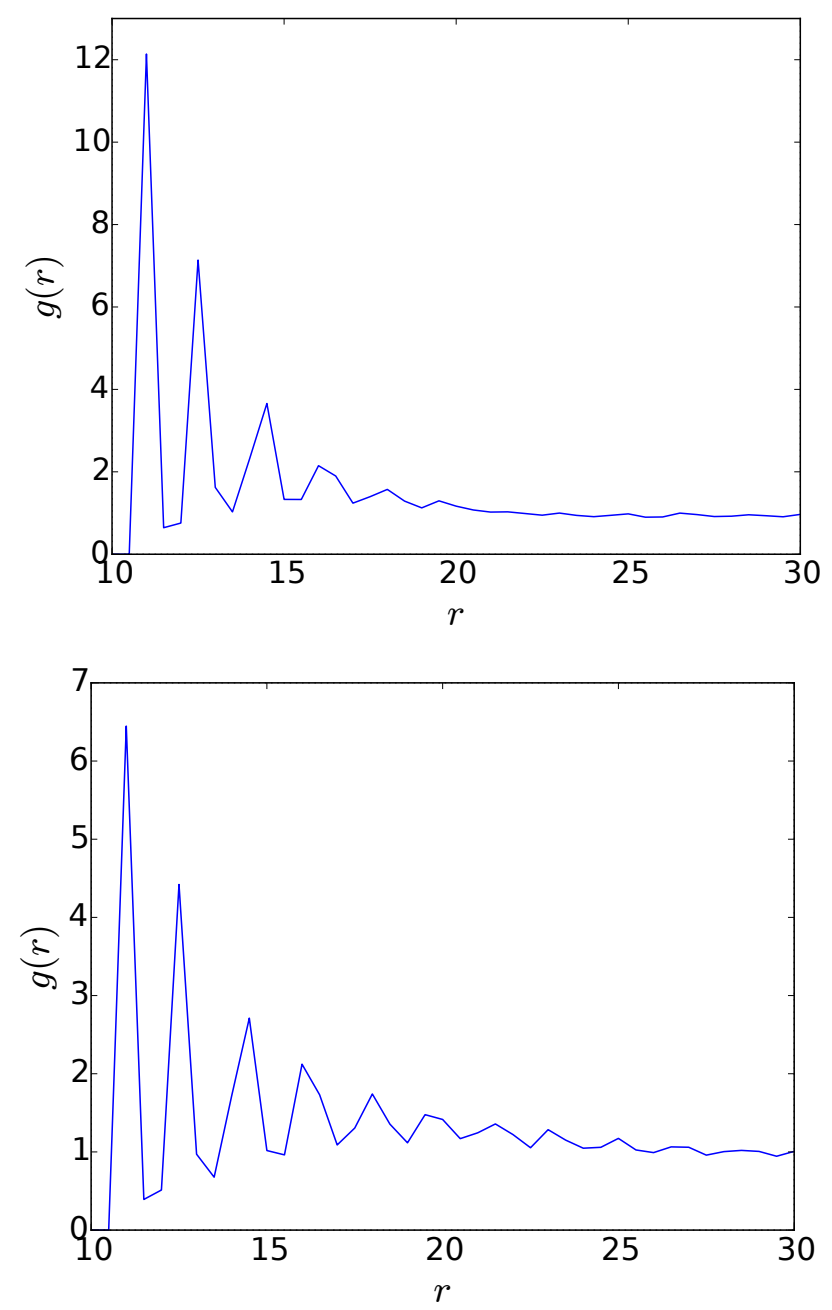

Figure 4.4: Obstacle-particle pair correlation function in systems with $y=$ $0.001, R_{p} / R_{o}=0.1, \phi_{o}=0.08$ and $\phi_{p}=0.2$ (top), $\phi_{p}=0.4$ (bottom). Increasingly more layers of particles accumulate around the obstacle by increasing the packing fraction.

As mentioned in chapter 1 , not only activity, but also the radius of the convex obstacles has been found to affect the particle trapping by the obstacles in systems where hydrodynamic interactions are dominant [53]. We also observe an increase of the residence times and the accumulation by enlarging the obstacle size (Fig. 4.5), simply because 


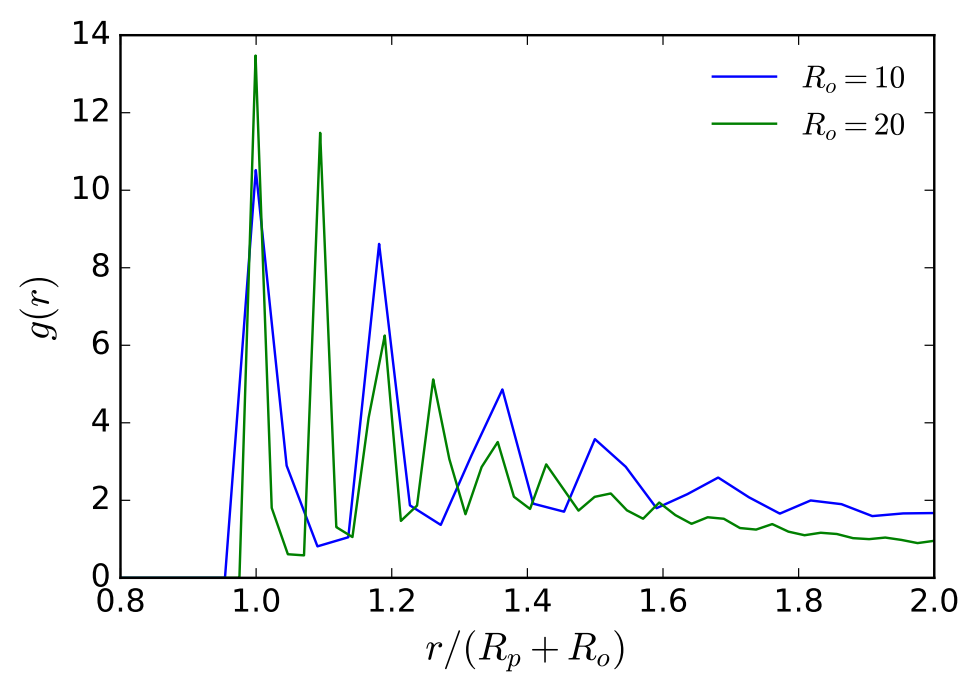

Figure 4.5: Obstacle-particle pair correlation function in systems with $y=$ $0.001, \phi_{p}=0.3, R_{p}=1$ and different obstacle radius $R_{o}$. The $x-$ axis is divided by $R_{p}+R_{o}$ to compare the first peaks of the two curves. The accumulation is enhanced by enlarging the obstacle.

in active systems (small rotational diffusion) the residence events are bounded by the finite size of the obstacle.

\subsection{CRYSTALLIZATION}

Systems in equilibrium (no self-propulsion) with short-range attractive and long-range repulsive interactions can exhibit clustering. Small clusters coarsen to form large ones and the system phase separates [104106]. While the details of such equilibrium clustering is known for more than a decade now, study of clustering in systems out of equilibrium is still in progress. Particularly systems with self-propulsion, which constantly uptake and dissipate energy and are hence out of equilibrium, have been explored by experiments, simulations and theory and shown to also exhibit clustering if the activity is sufficiently high $[34,69-71,73]$. The important distinction between this purely kinetic clustering and the analogue in equilibrium systems is the absence of attractive interactions in active systems. In other words, active systems with only repulsive interactions, can still exhibit clustering once the activity and density is sufficiently high. These clusters have been initially observed in elongated active particles where steric interactions can lead to mutual aligning of particles and thereby block their motion. However we know now that even purely repulsive spherical active systems with no aligning mechanism exhibit similar clustering.

At large densities the clusters show crystalline order. Experimental examples are hexagonal structures observed in catalytically-driven colloids [72] or vibrated monolayers of polar discs [75], and honeycomb- 
like clusters in colonies of flagellated marine bacteria [77]. Computer simulations have confirmed crystallization [69, 74] and theories have been proposed to describe this phenomenon $[76,78]$.

It is shown in the last section that in spite of no explicit attraction, particles tend to accumulate around obstacles below $y \approx 1$ (see Fig. 4.3) and form multiple layers as their density increases (Fig. 4.4). In fact for $\phi_{p}$ as large as 0.4 in a system with $\phi_{o}=0.08, R_{p} / R_{o}=0.1$ this effect is so strong that the system becomes heterogeneous, with dense clusters of particles around obstacles and a dilute gas of particles elsewhere (Fig. 4.6).
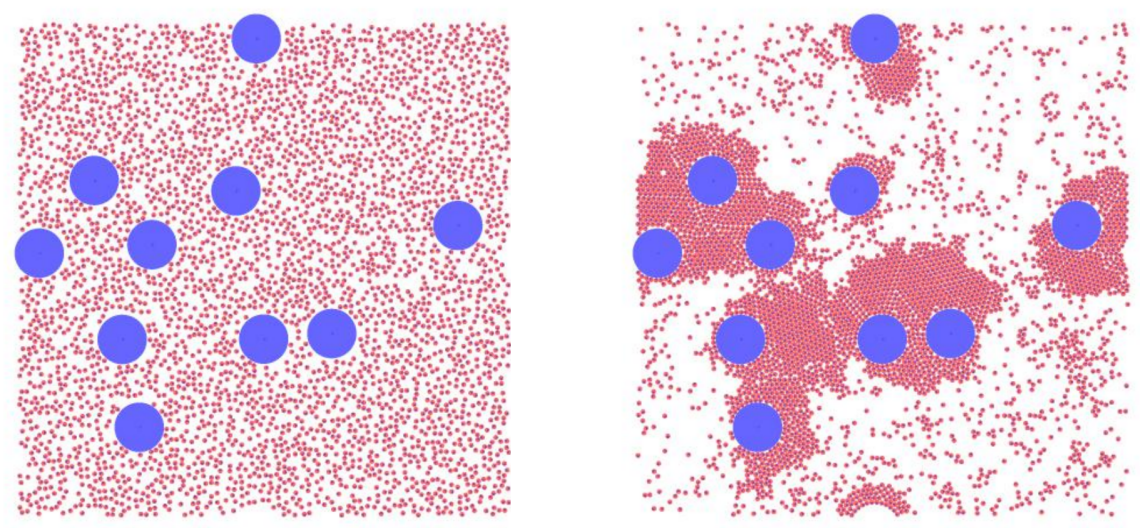

Figure 4.6: Development of crystals around the obstacles in a system with $\phi_{\mathrm{o}}=0.08, \phi_{\mathrm{p}}=0.3, R_{\mathrm{p}} / R_{\mathrm{o}}=0.1, x=0.1, y=0.001$ : figure in the left exhibits the initial configuration and figure in the right shows the configuration at some time in the steady state.

Our simulation results reveal that the formed clusters of particles are in fact hexagonal crystalline patches separated by grain boundaries. The structure factor,

$$
S(q)=\frac{1}{N} \sum_{i=1}^{N} \sum_{j=1}^{N} e^{-i \mathbf{q} \cdot\left(\mathbf{r}_{i}-\mathbf{r}_{j}\right)},
$$

for the $\mathrm{N}$ particles in a crystalline cluster around one of the obstacles (Fig. 4.7) is plotted in Fig. 4.8. This figure clearly reveals Bragg peaks of the hexagonal lattice. The magnitude of this reciprocal lattice vectors are found to be $\left|\mathrm{a}^{*}\right|=\left|\mathrm{b}^{*}\right| \approx 3.6$, which is in agreement with the real-space lattice vectors of magnitude $|a|=|b|=2 R_{p}=2$ (in a hexagonal lattice $\left|a^{*}\right|=\frac{2 \pi}{a} \sqrt{4 / 3}$ ). 


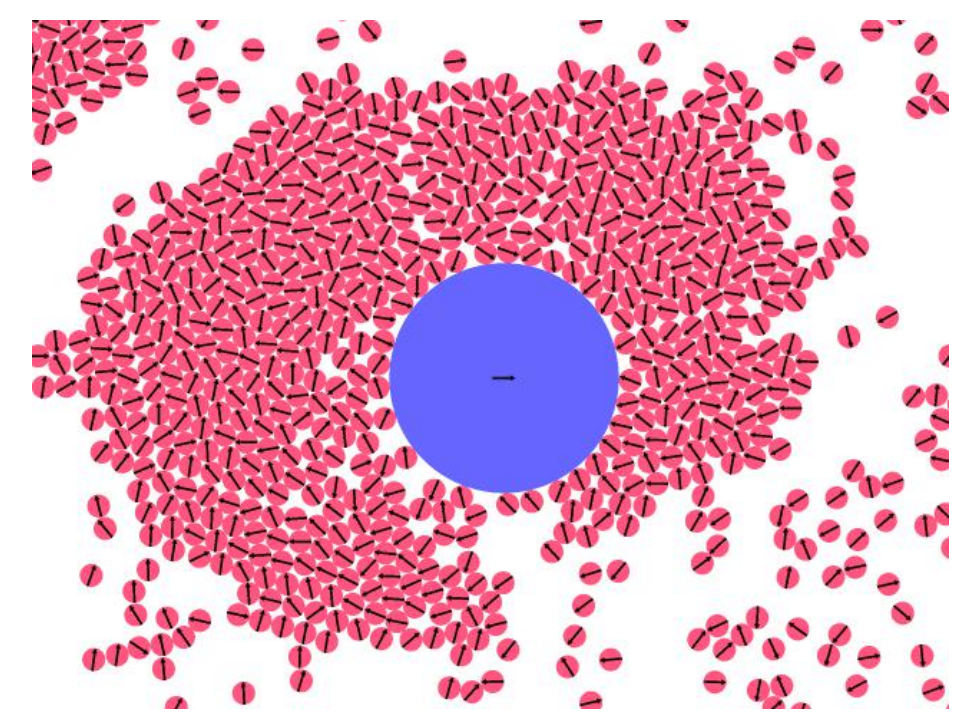

Figure 4.7: A snapshot of a system with $\phi_{\mathrm{o}}=0.08, \phi_{\mathrm{p}}=0.4, \mathrm{R}_{\mathrm{p}} / \mathrm{R}_{\mathrm{o}}=$ $0.1, x=0.1, y=0.001$. The aggregates on the obstacle exhibit crystallization with hexagonal patches separated by grain boundaries.

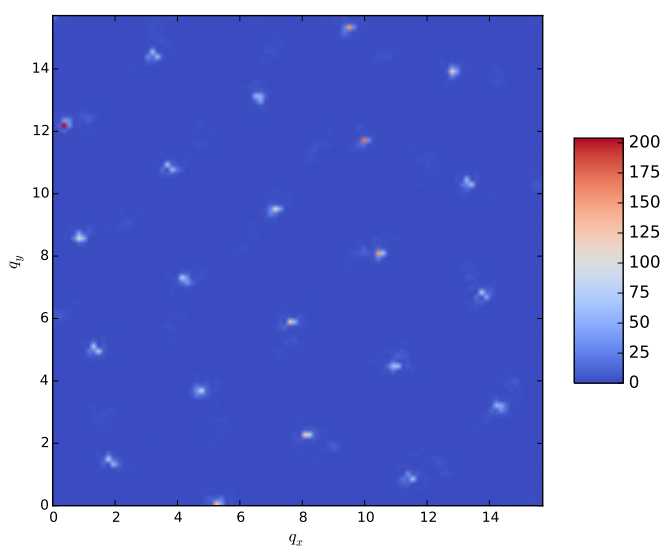

Figure 4.8: The structure factor calculated for particles in the crystal of Fig. 4.7 .

I have used a Delaunay triangulation to determine the boundaries of the crystalline cluster. Particles whose connecting edges are all smaller than some threshold, taken to be $2.1 R_{p}$, are considered inside the crystal and marked with red in the bottom part of Fig. 4.9. The bottom part of this figure illustrates a snapshot of the system under triangulation.

Particles with different orientations collide with the crystal and before they could turn around and escape it, they get trapped inside by other incoming particles. The orientation of particles in the crystalline state is therefore disordered and in general misaligned with their velocity. All the particles experience the same orienting force and try 

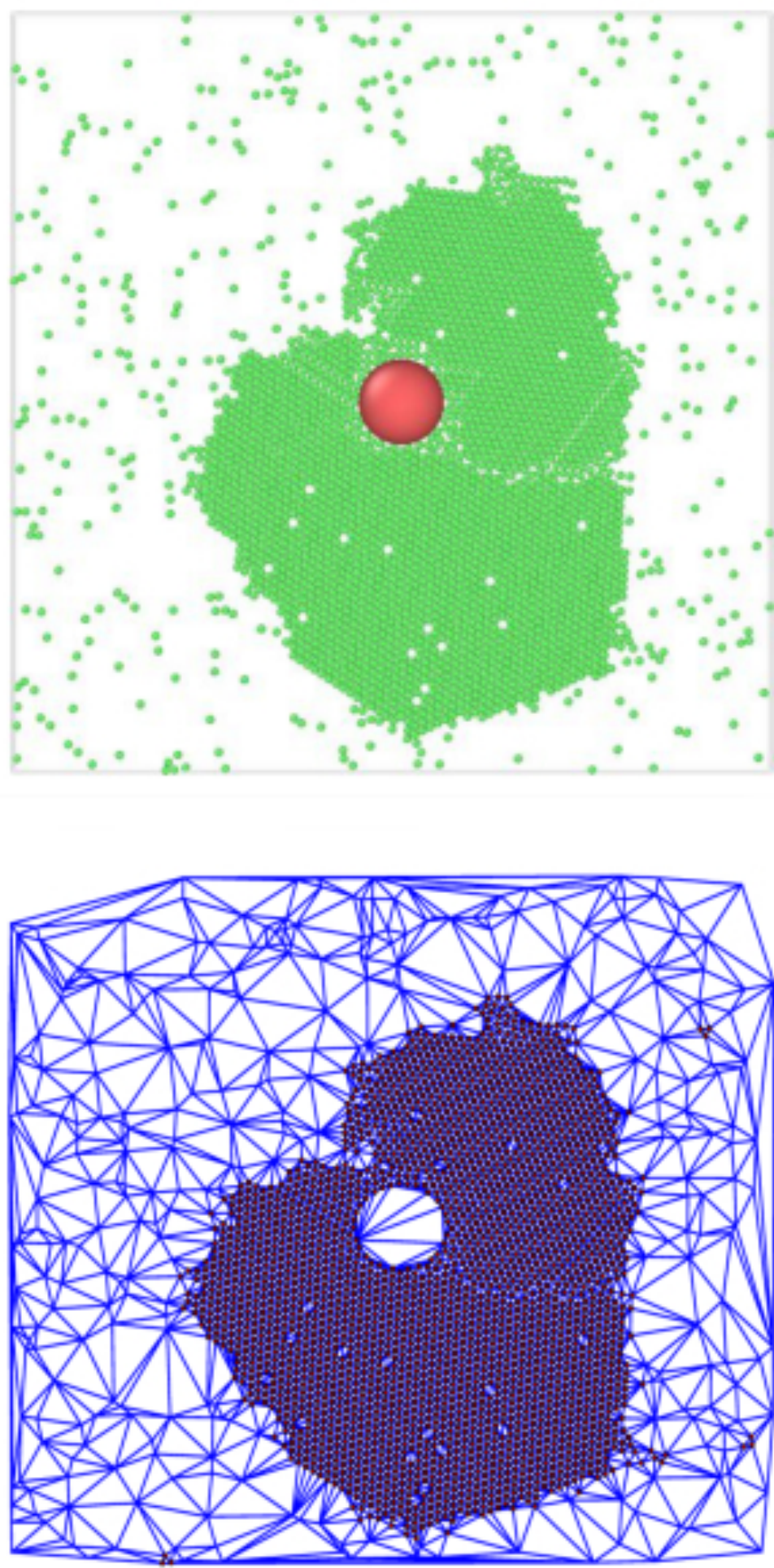

Figure 4.9: Snapshot of a system exhibiting a crystalline cluster around an obstacle (top) and the corresponding Delaunay triangulation of the system (bottom). Red particles in the triangulated picture, are vertices with all edges smaller than $2.1 R_{p}$. 


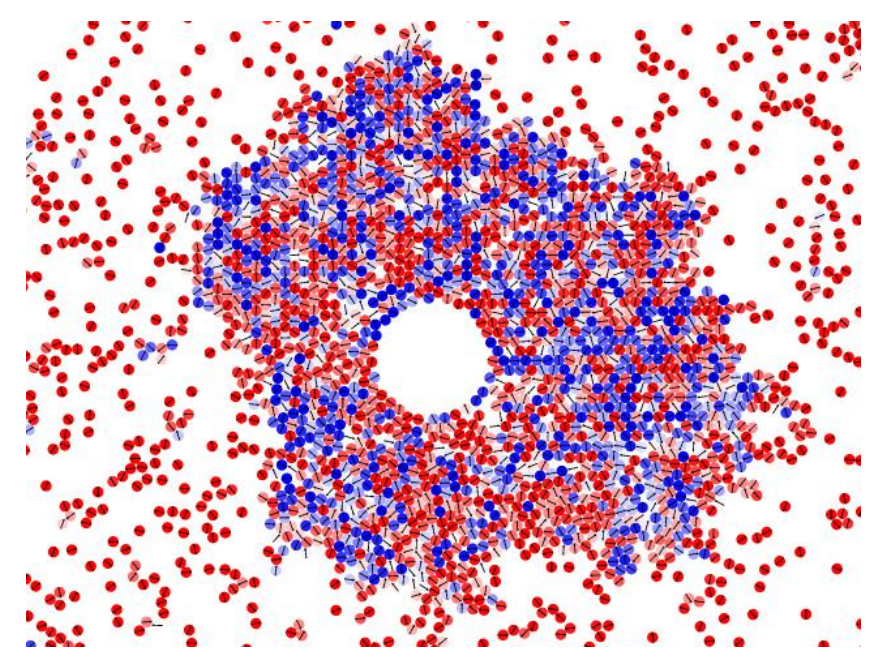

Figure 4.10: A crystalline cluster of particles formed around an obstacle $\left(R_{0}=10 R_{p}\right)$. Particles are color-coded according to their activity given by Eq. 2.27 from red ( $c=1$, or perfect alignment of velocity and orientation) to blue ( $c=-1$, or anti-alignment).

to align their velocity and orientation; however many collisions with nearby particles constantly randomize the velocity, while the orientation remains approximately fixed. We can conclude then that although high activity gives rise to accumulation and crystallization, the activity (alignment of velocity and orientation of particles, thanks to Eq. 2.27) is strongly suppressed in high density states. Fig. 4.10 illustrates the activity of individual particles inside a crystal around an obstacle in a system with $y=0.02$ and $\phi_{p}=0.3$. The crystalline state is also disordered with respect to the angular velocity for the same reason.

Crystallization occurs in active systems once the packing fraction of particles is sufficiently high. For a dilute system, even though active particles tend to be attracted to the obstacle, there is no large crowd of particles to give rise to accumulation and formation of crystals. As the activity is decreased, the effective attraction to the obstacle fades out and equilibrium crystallization occurs all over the system at dramatically larger density of particles. The range of parameters for which crystallization is observed, will be discussed in the next section.

The crystalline clusters around obstacles are not stationary: they form and grow, they may rotate or be frozen for a long time (this issue will be discussed in the next section), but they always dissolve and form again over the course of time. In order to study the stability of the crystalline clusters around obstacles, I have plotted the number of particles that are at the distance $r\left( \pm R_{p}\right)$ from an obstacle versus simulation time: Fig. 4.11. Our results in Fig. 4.11 are averaged over all the obstacles, and show that the first layers around obstacles will 


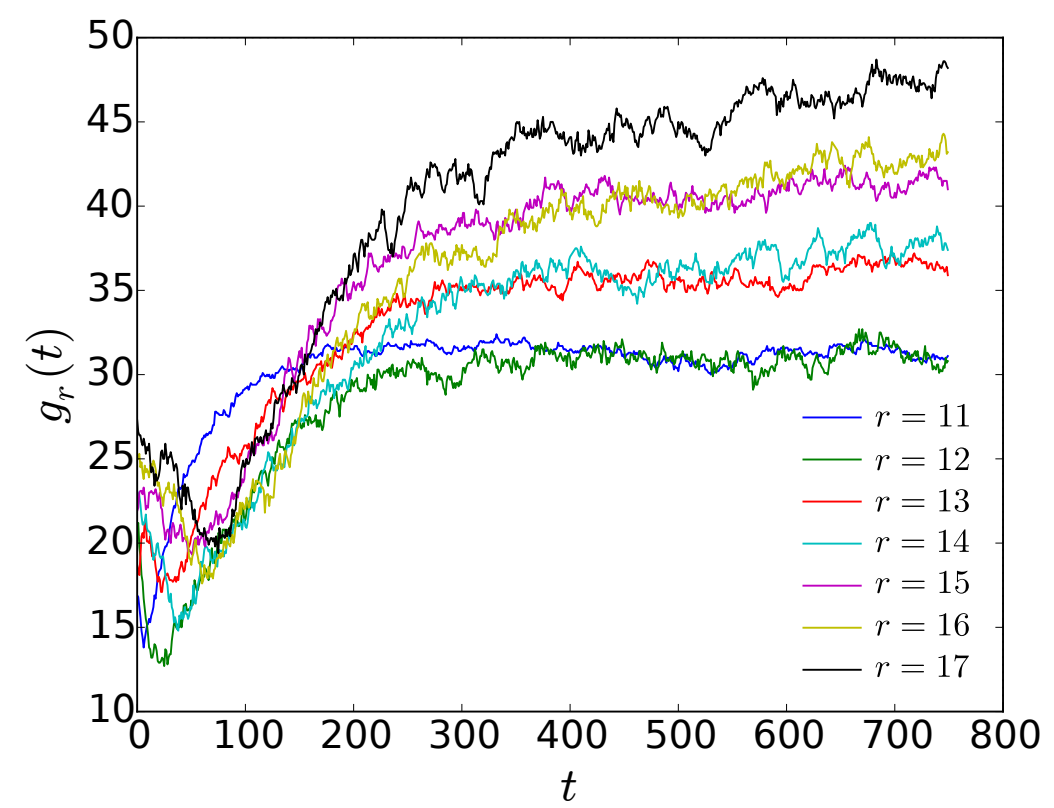

Figure 4.11: Number of the particles within shells of radius $r$ and thickness $R_{p}$ around an obstacle, averaged over all obstacles, for a system with $\phi_{o}=0.08, \phi_{p}=0.4, R_{p} / R_{o}=0.1, x=0.1, y=0.001$.

stay around the maximum they can get $\left(\mathrm{N}_{\mathrm{r}} \approx \frac{2 \pi r}{2 \mathrm{R}_{\mathrm{p}}}=\pi \mathrm{r}\right)$ over time. The ascending $g_{r}(t)$ for larger $r$ indicates the formation of more layers with time. Our simulation times in this figure are still not long enough to determine the stability of crystalline clusters.

However, longer times can be achieved by using the software, HOOMD (which I have shown to reproduce similar results as our program in chapter 2). Simulation results with HOOMD reveal that the crystalline structure can grow, dissolve, and re-grow again. In other words, once the system is active and crowded enough, it phase-separates into a crystalline solid and an active gas, with non-stationary boundaries. Fig. 4.12 shows a typical growth-dissolution cycle in an active crowded system.

\subsection{SPONTANEOUS ROTATIONS}

In the following section we show that active particles not only accumulate and crystallize around obstacles, but also the crystalline clusters perform collective rigid body rotations around them. Rotating aggregates of active particles have been previously observed in experiments $[80,83,84]$ and simulations [41, 81, 82]. In all these observations individual particles are either elongated or experience internal or external torque. In the former case, due to the steric interactions of aspherical particles, the spiral-like polarization field is quenched and hence the cluster may coherently rotate [81]. In the latter case, the rotation of individual particles is synchronized through hydrody- 

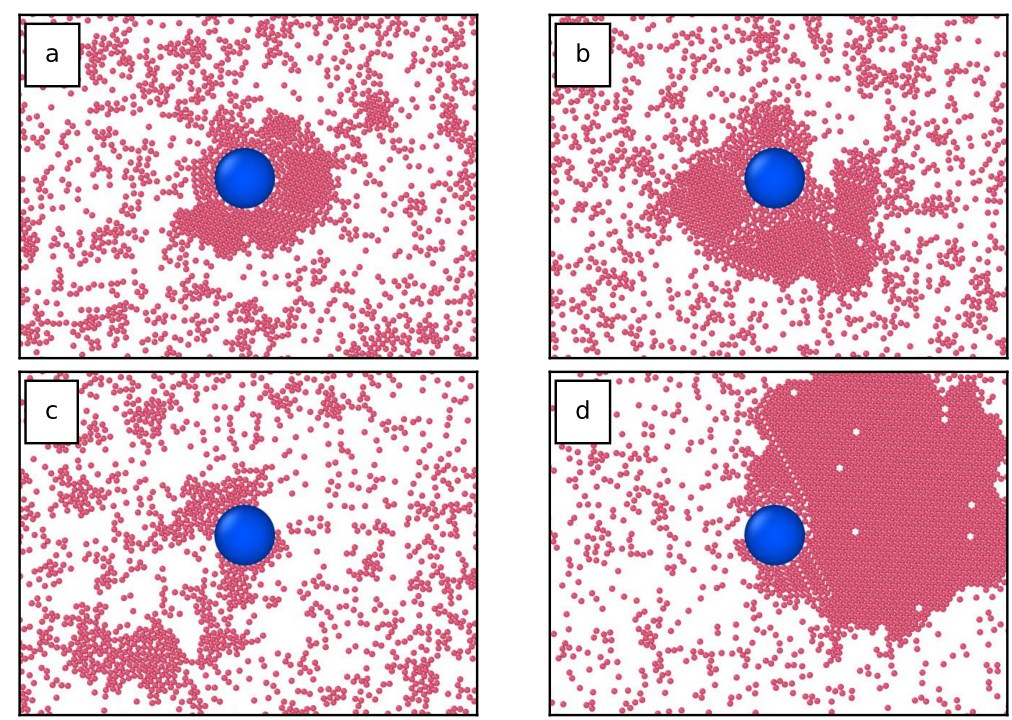

Figure 4.12: Growth, dissolution, and re-growth of the crystalline structure around the obstacle for a system with $\phi_{p}=0.3, y=0.001$. (a) to (d) represent the system's configuration at different times during a single run.

namic interactions and hence particles at the edge of the cluster give rise to a net rotation of the whole cluster [84]. Rotating clusters were claimed to be absent in systems of active spherical particles with repulsive pair potential [81].

Therefore the emergence of spontaneous rotations in systems of active spherical particles with linear propulsion only, is an interesting phenomenon which will be described and explained below.

\subsubsection{Observation}

Fig. 4.13 illustrates the formation and motion of a rotating crystal. Particles in this figure are color-coded according to the direction of the component of their velocity along $\mathbf{e}_{\phi}$, where $\mathbf{e}_{\phi}$ is the tangential vector to the connecting line of the particle and obstacle. For a clockwise $(\mathrm{CW})$ rotating crystal I take

$$
\mathbf{e}_{\phi}=(-\Delta y, \Delta x) \frac{1}{\sqrt{\Delta x^{2}+\Delta y^{2}}},
$$

where $(\Delta x, \Delta y)$ refers to the vector connecting the particle to the obstacle $\left(\Delta x=x_{0}-x_{p}\right)$. For a CCW rotating crystal, the direction of $\mathbf{e}_{\phi}$ is the opposite of the above expression. This figure demonstrates the onset of rotation of a crystal: the velocity of all particles in the crystal has a positive component tangent to the direction of rotation of the whole crystal and the crystal begins to rotate as a whole. 

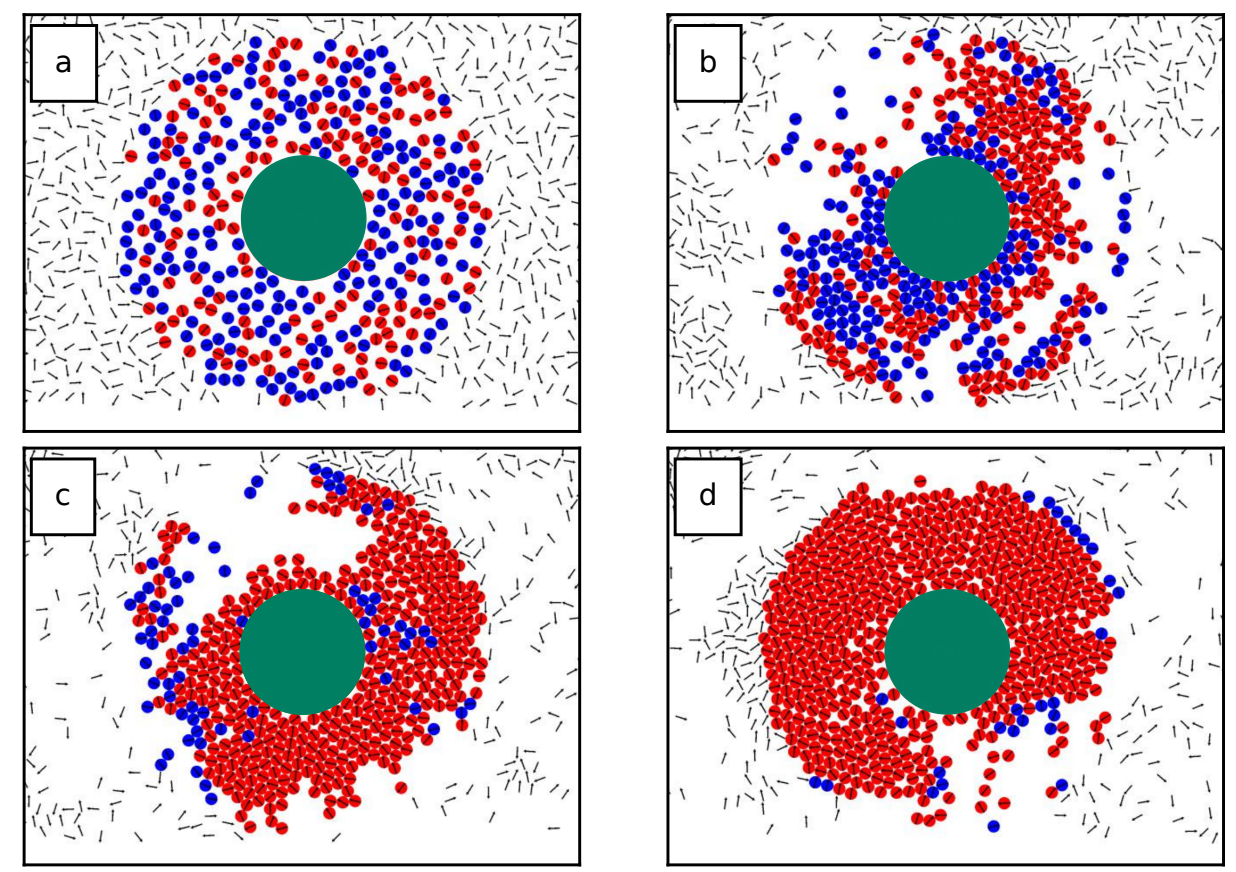

Figure 4.13: Formation of a CW rotating crystal from (a) to (d). Particles are color-coded according to the orientation of their velocities: red corresponds to $\mathrm{CW}$, and blue corresponds to CCW motion around the obstacle.

The angular velocity and orientation of individual particles do not exhibit the same uniform collective behavior as $v_{\phi}$ (Fig. 4.14) as particles do not exchange information about their orientation or angular velocity through their collisions. This however, does not necessarily imply a uniform distribution of the orientation of particles in a rotating crystal: we can address this issue by studying the activity of individual particles trapped in a rotating crystal.

Particles in Fig. 4.15 are color-coded according to their instantaneous activity (see our definition of activity in eq. 2.27),

$$
\mathrm{c}=\frac{\mathbf{v} \cdot \mathbf{n}}{|\mathbf{v}|}
$$

from blue $(c=-1)$ to red $(c=1)$. This figure exhibit the $C W$ rotation of a growing crystal around the obstacle. One can divide the whole crystal into many slices and measure the average activity of particles, $c(\phi)$, in each of these slices, located roughly at an angle $\phi$ relative to the obstacle. I further average $c(\phi)$ over many snapshots that capture the same rotation of the crystal around the obstacle (note that in order to add the results of different snapshots together, I choose the point of reference in measuring the angle $\phi$ always at the "back" of the rotating crystal in different snapshots, so that $\phi=0$ refers to the back and the largest $\phi$ refers to the front of the crystal). Fig. 4.16 reveals that this quantity has a gradient along the direction of rotation, 


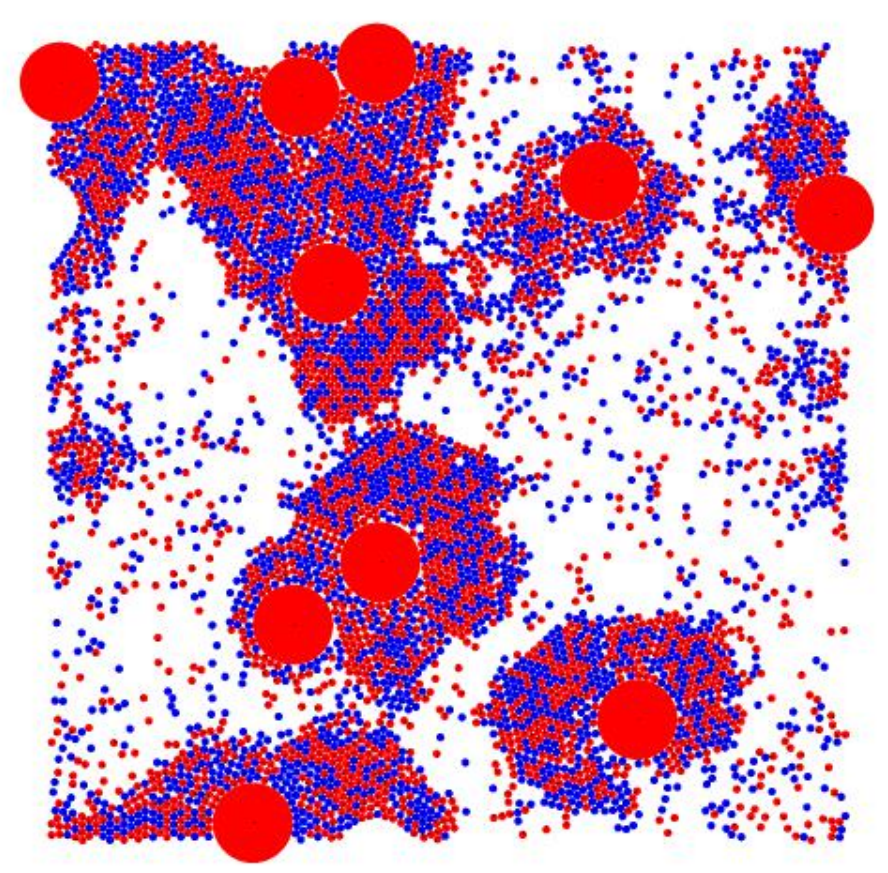

Figure 4.14: The direction of angular velocity is color-coded. There is no particular order in this quantity in the rotating crystals. This is expected, as collisions do not affect $\omega$.

whereas no such gradient can be observed in a non-rotating crystal (Fig. 4.17 and 4.18).

The rigid-body rotation is more clearly illustrated in Fig. 4.19. The color coding is based on $v_{\phi}=\mathbf{v} \cdot \mathbf{e}_{\phi}$. The initially formed crystal in Fig. 4.19a) starts to rotate while growing and soon all the particles in the cluster acquire a positive $v_{\phi}$. Comparing Fig. 4.19c) to Fig. 4.19b) reveals that the rotating velocity of the crystal is enhanced in time (particles get more red). The increase of the rotational velocity is widespread in all our rotating clusters and will be explained below. Eventually in Fig. 4.19d) the crystal starts to dissolve and the rotation decays. The marked chain of particles in dark green illustrates a rigid-body rotation of the cluster by about $\pi / 2$. The cluster is stable as long as its opposite sides "push towards each other". Since the rotating crystals are observed in the limit of high activity, where the persistence time of particle's orientation is long, this implies that the crystal is stable for a rotation by roughly $\pi / 2$; above this value different ends of the crystal move in different directions and it dissolves. This is also a reason for why rotations are only observed at large activities: the persistence time of orientations $(\propto 1 / y)$ has to be larger than the lifetime of a crystal, in order to observe the full rotation. The hexagonal crystals around obstacles can grow surprisingly large while they rotate, for instance the cluster in Fig. 4.19 consists of 


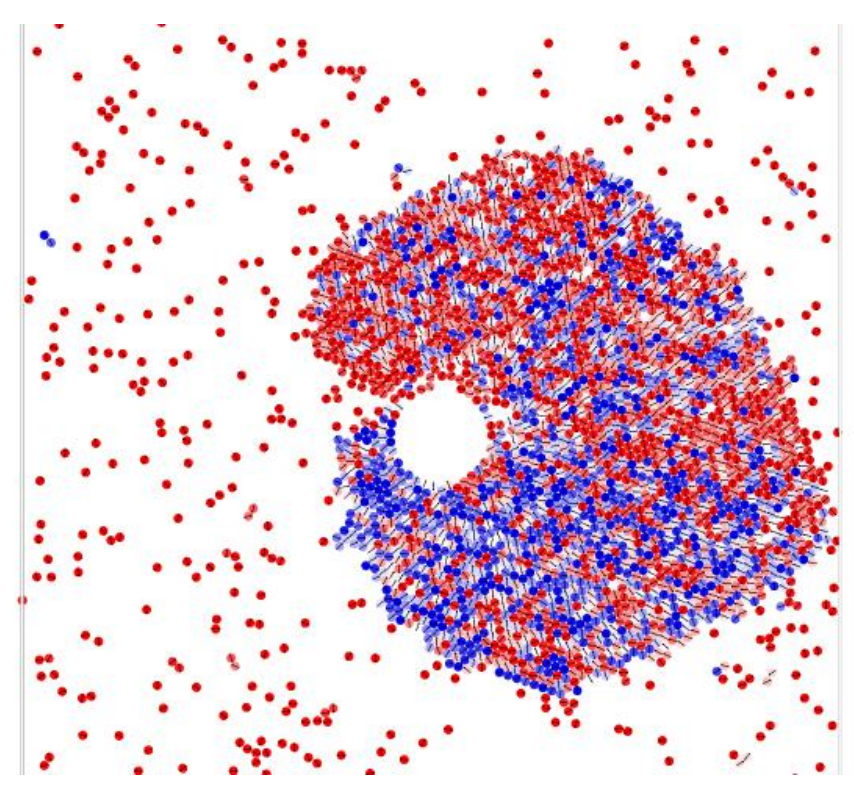

Figure 4.15: A CW rotating growing crystal of particles around an obstacle of size $R_{0}=10 R_{p}$. The activity of particles is color-coded: red and blue correspond to $c=1$ and -1 respectively. Particles on the back of the rotating crystal tend to move almost along their orientations, whereas particles on the front are mostly pushed by the crystal opposite to their orientations.
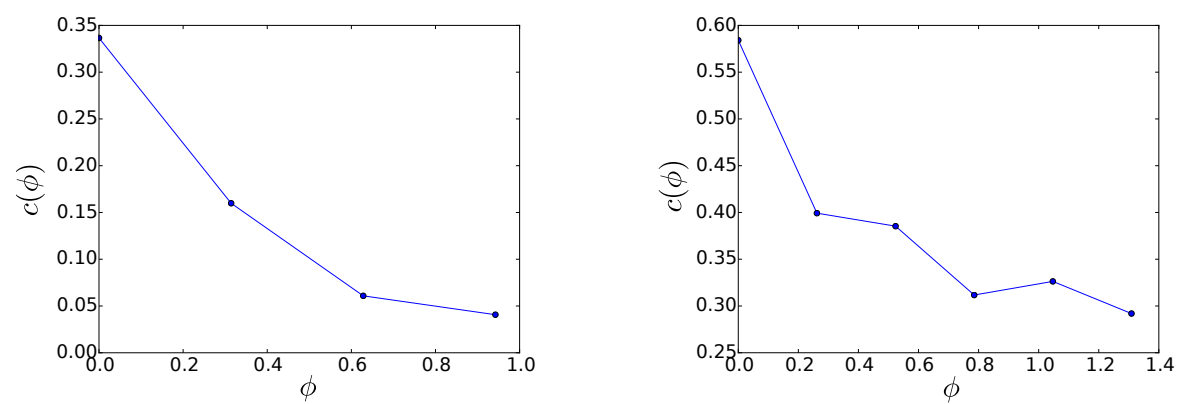

Figure 4.16: Gradient of local activity in two different rotating crystals, averaged over 80 snapshots. $y=0.001$ and $\phi$ is in radians.

$\mathrm{N} \approx 3000$ particles at its largest stage.

\subsubsection{Derivation of the rotations}

Now I present a simple argument to explain our observations of rotating crystals. We aim to understand the mechanism that gives rise to the buildup of systematic rotations once a spontaneous fluctuation has occurred. A simple model of a rough, moving wall is presented below. I show that particles with different velocities (relative to the velocity of the wall) have different chances of being attracted to the wall. The wall acquires increasingly more particles with velocities along the 


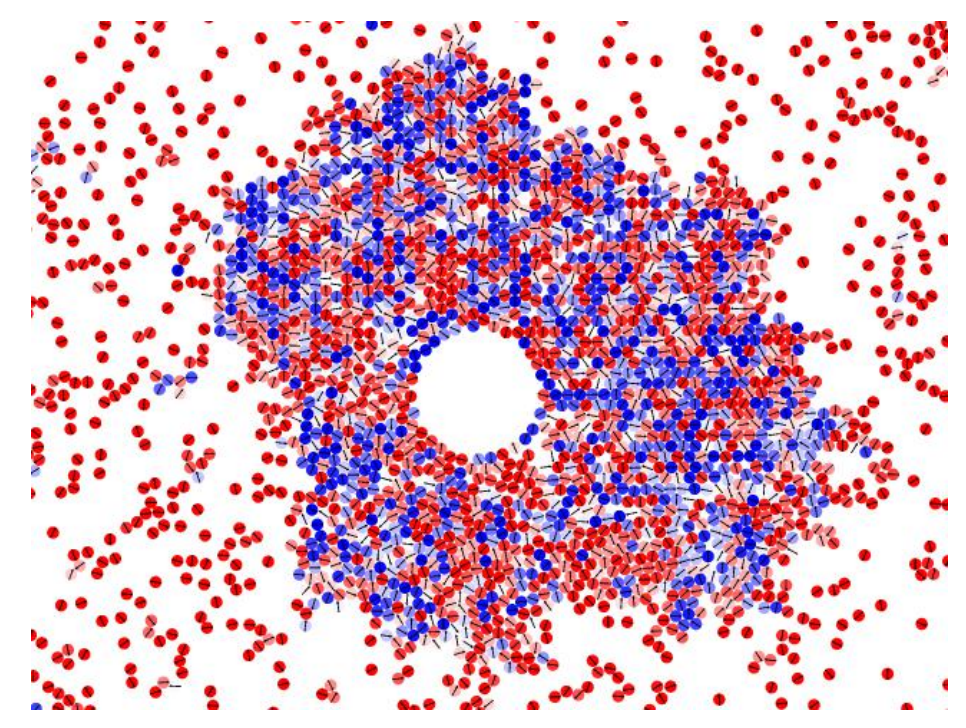

Figure 4.17: Demonstration of a non-rotating crystal in a system with $\phi_{p}=0.3, y=0.01$. Color-coding is based on the activity of particles.

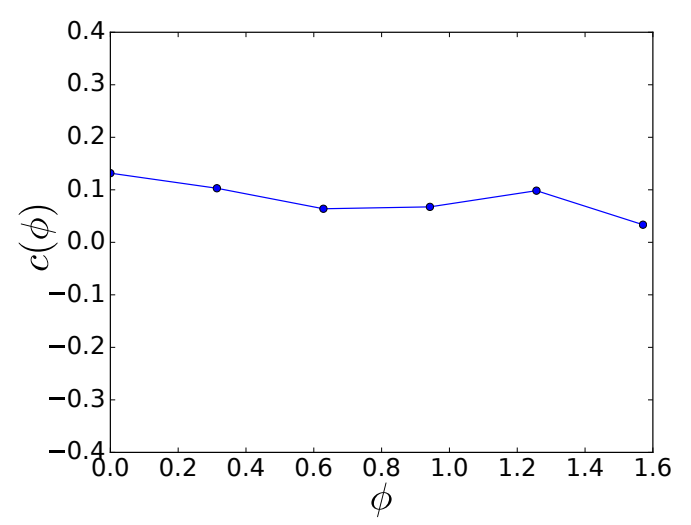

Figure 4.18: Gradient of activity in the crystal shown in Fig. 4.17. The local activity is homogeneous through the entire non-rotating crystal.

wall's velocity as compared to particles with opposite velocities.

We consider the rough moving wall with velocity $\mathbf{v}_{0}$ as a flat wall adorned with triangular spikes to simulate roughness (Fig. 4.20). A particle that collides with the wall at time $t=0$ is elastically reflected and its velocity will be $\mathbf{v}^{\prime}=\mathbf{v}^{s}-2 \mathbf{n}_{w}\left(\mathbf{n}_{w} \cdot\left(\mathbf{v}^{s}-\mathbf{v}_{0}\right)\right)$, where $\mathbf{n}_{w}$ is the normal to the wall at the point of collision and $\mathbf{v}^{s}$ is the velocity of the particle in the stationary state.

Since rotations occur for highly active systems only, where rotational diffusion is slow on the timescales under consideration, we assume that the equations of motion (Eq.2.9a and 2.9b) can be simplified to

$$
\frac{d}{d t} \mathbf{v}=-\mathbf{v}+\sqrt{x} \mathbf{n},
$$



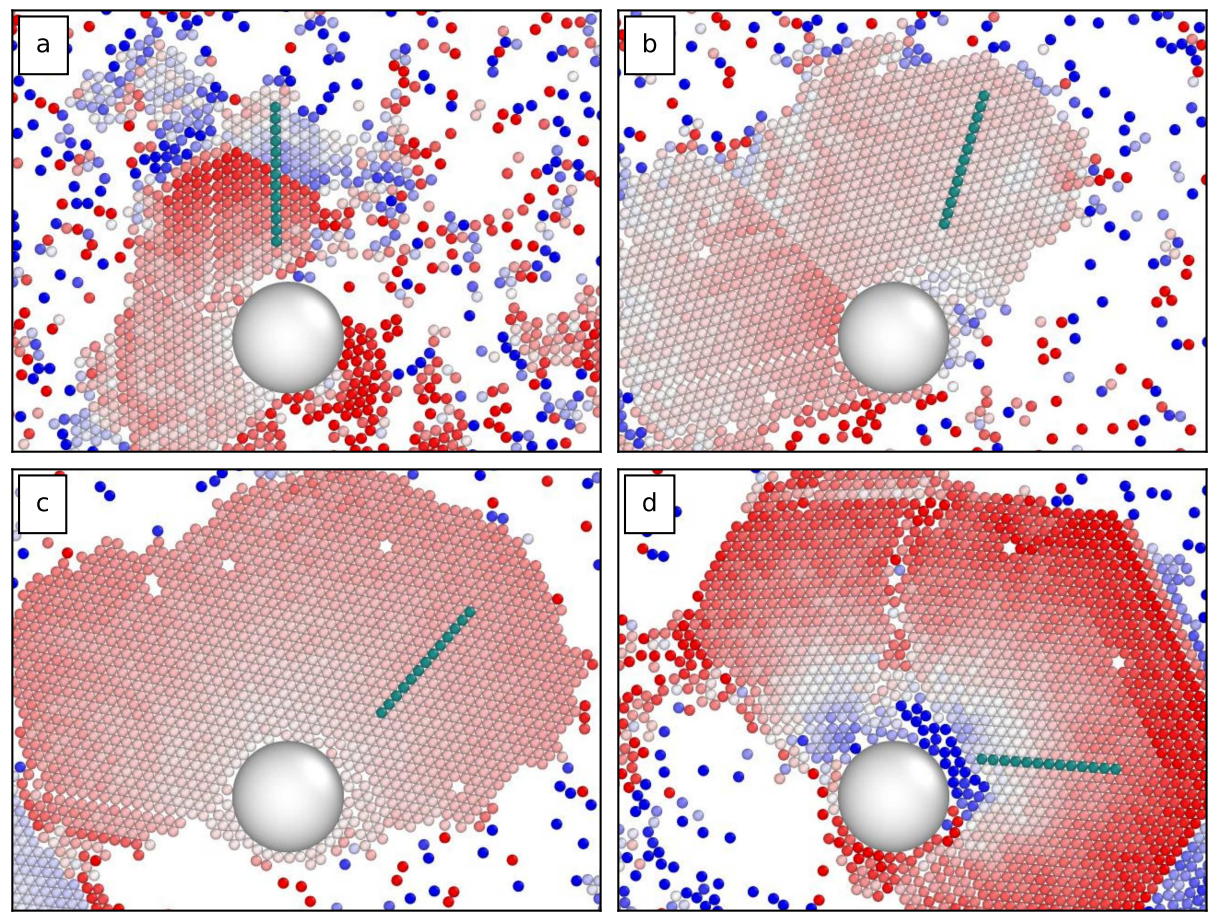

Figure 4.19: Formation of a CW rotating crystal. Particles are color-coding according to their $\mathbf{v}_{\phi}$ : the spectrum from red to blue corresponds to CW to CCW motion around the obstacle respectively. Particles marked in green are to help visualizing the rigid body rotation of the cluster from snapshot a to $d$.

giving rise to a stationary velocity $\mathbf{v}^{s}=\mathbf{n} \sqrt{x}$. Now the above equation can be solved to find the distance to the base of the wall in the next collision with the wall at time $t_{1}$ :

$$
y\left(t_{1} ; \mathbf{v}_{0}\right)=y\left(t_{1} ; 0\right)+\left(\mathbf{e}_{y} \cdot \mathbf{n}_{w}\right)\left(\mathbf{n}_{w} \cdot \mathbf{v}_{0}\right) t_{1} .
$$

The above equation reveals that if the wall is moving with a velocity parallel to the particle's velocity, then the particle will hit the moving wall deeper inside in its next collision, as compared to the case where the wall moves anti-parallel to the particle's velocity. $\mathbf{e}_{y} \cdot \mathbf{n}_{w}>$ 0 for all collisions, but in the former case $\mathbf{n}_{w} \cdot \mathbf{v}_{0}<0$ and hence $y\left(t_{1} ; v_{0}\right)<y\left(t_{1} ; 0\right)$; in other word the particle moves closer to the baseline. Whereas for the latter case $y\left(t_{1} ; v_{0}\right)>y\left(t_{1} ; 0\right)$ and the particle may slip away from the wall.

This argument is given for a flat wall but is expected to hold for a curved wall as well, if the curvature is sufficiently small, or in other words if the cluster's extension is large compared to the radius of the particles. For our case of crystallization on obstacles, $\mathbf{v}_{0}$ is approximated by $\Omega \times \mathbf{R}$ where $\Omega$ is the cluster's rotational velocity and $\mathbf{R}$ is the connecting vector from a particle on the edge of the cluster to the center of the obstacle (magnitude of $\mathbf{R}$ denotes the radius of the clus- 


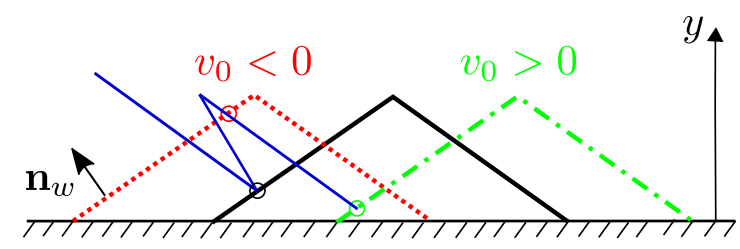

Figure 4.20: Sketch of a sample trajectory of a particle in two successive collisions with a model rough moving wall.

ter). For a particle approaching a rotating cluster then, the probability of attaching to it given that it has a velocity parallel to $\Omega \times \mathbf{R}$ at that point $\left(p_{+}\right)$is larger than the anti-parallel alternative $\left(p_{-}\right)$. Since the alignment between velocity and orientation of active particles is almost perfect, we can consider the orientation of particles that join the cluster, instead of their velocity. Now we introduce the quantity $\mathrm{N}_{+}$ and $\mathrm{N}_{-}$which measure the number of particles in the cluster with orientation parallel or antiparallel to $\Omega \times \mathbf{R}$ respectively. The rate of change of this quantity is given by

$$
\frac{d N_{ \pm}}{d t}=p_{ \pm} j 2 \pi R \quad \text { with } \quad p_{+}>p_{-}
$$

where $j$ is the flux along the contour of $2 \pi R$. To find $N_{ \pm}$as a function of time, note that the area of the cluster is approximately given by $\pi R^{2}=V_{o}+V_{p}\left(N_{+}+N_{-}\right)$, where $V_{o}$ and $V_{p}$ are the volume of the obstacle and a single particle respectively. Hence

$$
2 \pi R \frac{d R}{d t}=V_{p}\left(\frac{d N_{+}}{d t}+\frac{d N_{-}}{d t}\right)=V_{p} 2 \pi R j\left(p_{+}+p_{-}\right) .
$$

Therefore at the beginning of the growth of the cluster, when $p_{ \pm}$ can still be assumed constant, the radius grows linearly with time: $R(t)=R(0)+V_{p} j\left(p_{+}+p_{-}\right) t$ and as a result of that and Eq. 4.9,

$$
N_{ \pm}(t)=N_{ \pm}(0)+p_{ \pm} j 2 \pi R(0) t+\mathcal{O}\left(t^{2}\right) .
$$

The linear growth of $\mathrm{N}_{+}$and $\mathrm{N}_{-}$at small times is indeed observed for small times in our simulations (see the left column of Fig. 4.21). At larger times, once the cluster acquires a substantial rotating velocity, we expect $p_{+}-p_{-}$to increase with time: Our arguments for the rough moving wall above suggest that $p_{+}$and $p_{-}$depend on the relative velocity of the approaching particle and the cluster, and we have observed in our simulations that $\Omega$ grows in time. The relative velocity of the particle with anti-parallel orientation to the cluster's rotating direction then increases in the course of time (and decreases for a particle with parallel orientation), yielding an increase in $p_{+}-p_{-}$as a function of time. Hence $\mathrm{N}_{+}$increases more strongly as the time goes by, while counterrotating particle hardly have a chance to be captured. In other words $\mathrm{N}_{-}$keeps increasing with a smaller and smaller rate, until it almost saturates. These predictions that are based on a simple 
model for the rough moving wall are confirmed by our simulations, presented in the left column of Fig. 4.21. $\mathrm{N}_{+}$and $\mathrm{N}_{-}$grow similarly due to finite and initially equal values of $p_{+}$and $p_{-}$(see Eq. 4.9). However they spread at some time (this time coincides well with the beginning of the rotation of the cluster and will be explained below): $\mathrm{N}_{+}$increases more dramatically until the cluster dissolves and the rotation ceases, while the rate of increase of $\mathrm{N}_{-}$decreases and it saturates.
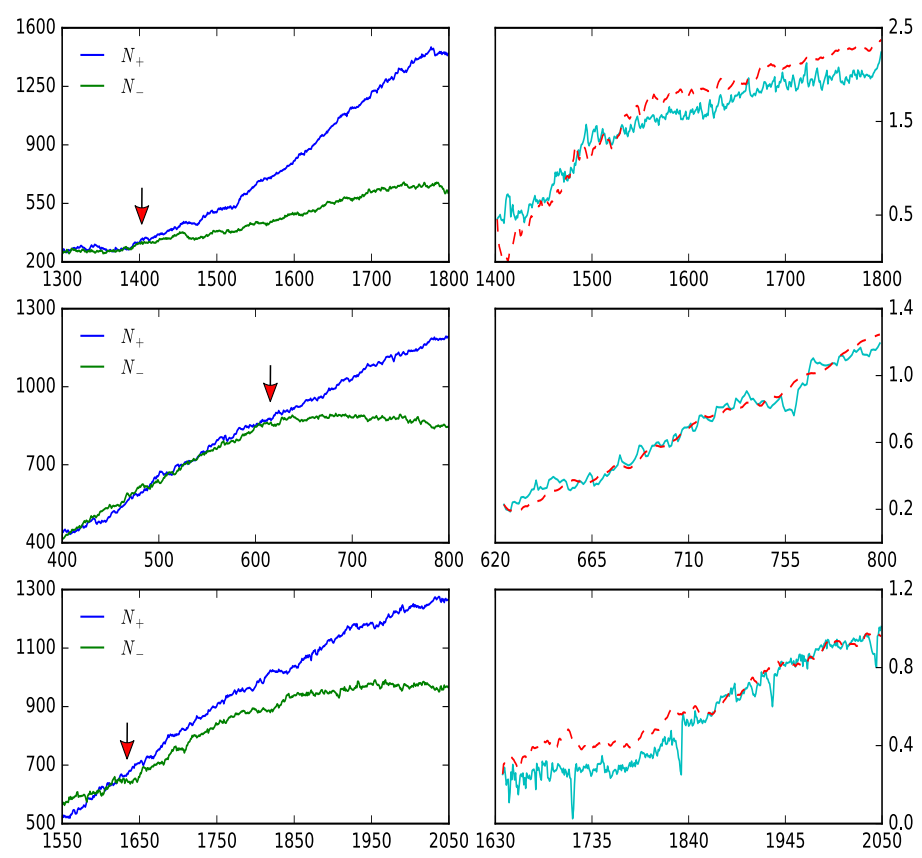

Figure 4.21: Left: $N_{ \pm}$versus time in several clusters. Red arrows indicate the time when $\frac{\mathrm{N}_{+}-\mathrm{N}_{-}}{\mathrm{N}}>0.03$; we mark the onset of rotation by this time. Right: Rotational velocity of the clusters, $\Omega \times 10^{3}$, corresponding to the left columns. Data from simulations are compared to the result of integration of Eq. 4.10 (dashed lines).

We have so far only observed the increase of the cluster's angular velocity in the simulations. But can we also track that down to our equations of motion? I answer this question in the following.

We assume the cluster performs a rigid-body rotation so that the velocity of the ith particle is $\mathbf{v}_{i}=\Omega \times \mathbf{r}_{i}$, where $\mathbf{r}_{i}$ is the vector connecting the center of the obstacle to the $i$ th particle. Now in the limit of high activity we may use Eq. 4.5 for the cross-product of $\mathbf{v}_{i}$ and $\mathbf{r}_{i}$ and sum over all particles in the cluster to find

$$
\mathrm{I}_{\mathrm{cl}} \frac{\mathrm{d}}{\mathrm{dt}} \boldsymbol{\Omega}=-\mathrm{I}_{\mathrm{cl}} \boldsymbol{\Omega}+\sqrt{x} \sum_{\mathrm{i}} \mathbf{r}_{\mathrm{i}} \times \mathbf{n}_{\mathrm{i}}
$$

Here $I_{c l}=\sum_{i} r_{i}^{2}$ is the moment of inertia of the cluster. This equation shows that the imbalance between $\mathrm{N}_{+}$and $\mathrm{N}_{-}$causes a torque 
$M=\sqrt{\chi} \sum_{i} \mathbf{r}_{i} \times \mathbf{n}_{i}$ which has to overcome the damping for spontaneous rotations to emerge. One the rotation starts, the torque increases due to increasing imbalance of the number of particles with positive and negative $\mathbf{r}_{\boldsymbol{i}} \times \mathbf{n}_{i}$. Numerical integration of Eq. 4.10 reveals an increase of $\Omega$ which compares well with our simulations (right column of Fig. 4.21).

To summarize the few lines above, we have leaned that the spontaneous rotations of active crystals around a large static obstacle can be regarded as a rigid body rotation, whose onset is well indicated by the excess of $N_{+}$over $N_{-}$. This imbalance is further enhanced by an increasing $\Omega$, providing a feedback mechanism for the observed systematic rotations.

Now before moving on to investigating the range of parameters for which rotating crystals may appear, an important distinction between our observation and other rotating active aggregates needs to be pointed out. In [80] for example, single particles are added randomly to the cluster and hence the summation of the individual torques they insert on the cluster scales as the square root of their total number. On the contrary we observe a linear dependence of the total torque on the number of particles in the cluster (Fig. 4.22A). This can be understood through approximating $M=\sqrt{x} \sum_{i=1}^{N} \mathbf{r}_{i} \times \mathbf{n}_{i}$ by $M=\sqrt{x}\left(\sum_{i=1}^{N_{+}} \mathbf{r}_{i}-\sum_{i=1}^{N_{-}} \mathbf{r}_{i}\right)$. It is clear now that $M \sim N_{+}-N_{-}$and since $\mathrm{N}_{\text {- }}$ saturates soon after the rotation is established, one can conclude that $M \sim N$. It is also shown in Fig. 4.22C how the rotational velocity of the cluster initially fluctuates, before it starts to grow systematically and $\mathrm{N}_{+}-\mathrm{N}_{-}$scales like $\mathrm{N}$.

\subsubsection{Phase diagram}

Here I raise the following question: How does the existence of the rotating crystals depend on our model parameters? Particularly how active and dense does a system need to be in order to observe the rotating crystals? How does the system look like for other values of activity and packing fraction?

We quantify the rotation of a crystal by the angular velocity of particles around the obstacle,

$$
\omega_{i}^{c}=\frac{\mathbf{v}_{i} \cdot \mathbf{e}_{\phi}}{r_{i}} .
$$

Distribution of this quantity is plotted in Fig. 4.23 for different values of $y$ for a system with one obstacle and particle density $\phi_{p}=0.4$. The positive and negative values of $\omega_{\phi}$ correspond to CW and CCW rotation respectively. 

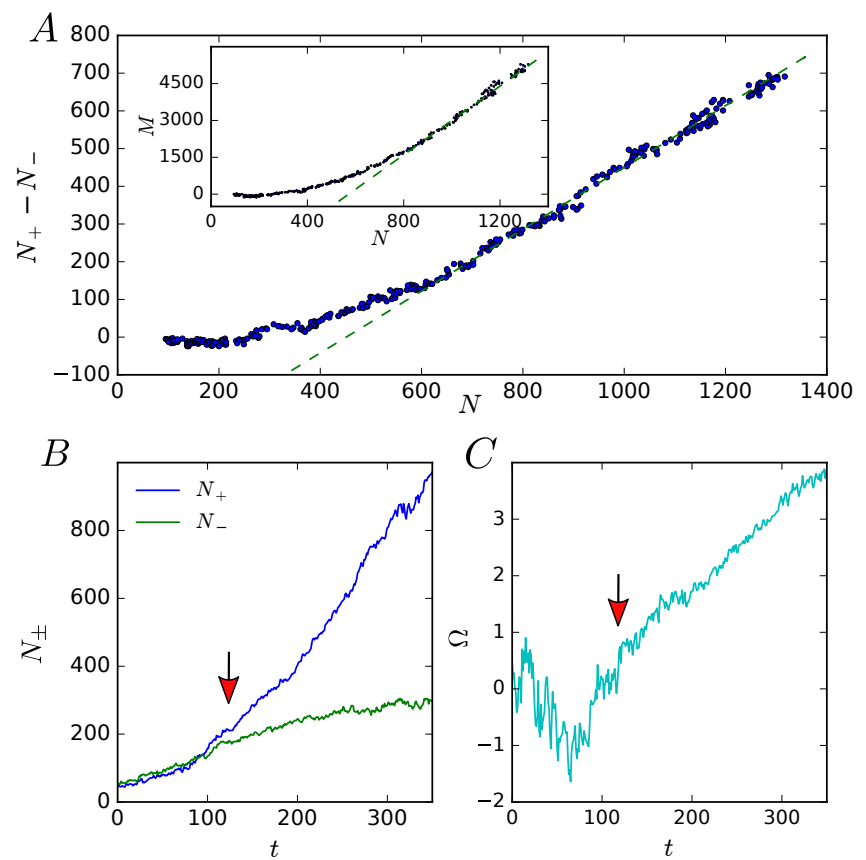

Figure 4.22: $A: N_{+}-N_{-}$versus total number of particles $N$ in a cluster as it grows, observed in a system with $y=0.001, \phi_{p}=0.3$; inset: the torque $\mathrm{M}$ as a function of $\mathrm{N}$; $\mathrm{B}$ : growth of $\mathrm{N}_{+}$and $\mathrm{N}_{-}$with time; C: Change of $\Omega$ with time; both (B and $C$ ) for the same cluster as in $\mathrm{A}$. The onset of the rotation is marked by the red arrow.

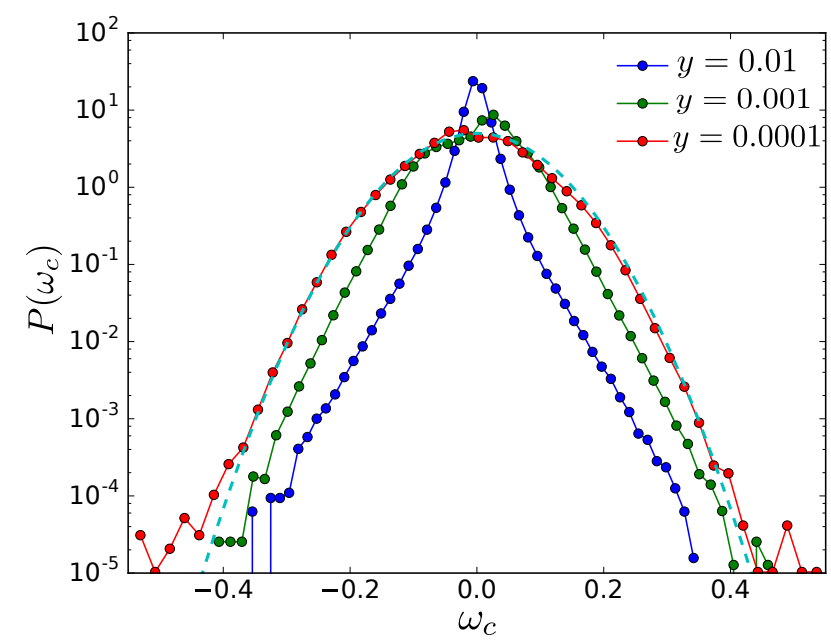

Figure 4.23: Distribution of angular velocity of particles around the obstacle. $\phi_{p}=0.4$. The dashed line fits a Gaussian to the red curve.

Consulting our simulation videos, rotating crystals only appear for very active systems and at some intermediate densities, above which the system-spanning crystal does not allow any rotation, and below which the number of accumulated particles around the obstacle does 


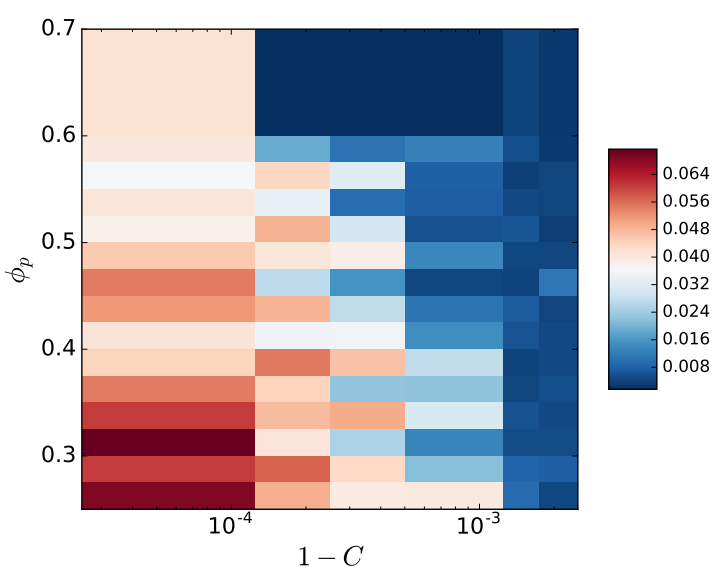

Figure 4.24: Average magnitude of the angular velocity of the crystal around the obstacle, $\Omega$, as a function of particle activity and packing fraction.

not suffice to form a stable-enough crystal. Fig. 4.24 illustrates the magnitude of average angular velocity of particles (what we call the cluster's rotational velocity) around the crystal,

$$
\Omega=\left|\frac{1}{N} \Sigma_{i=1}^{N} \omega_{i}^{c}\right|,
$$

for different values of particle activity and packing fraction. Here $\mathrm{N}$ is the number of particles in the crystal around the obstacle. The statistics are improved by averaging this quantity over different snapshots. The activity of particles is tuned with $\mathrm{y}$. Note that the average particle's activity can be estimated as $C=\sqrt{\frac{1}{1+y / 2}}$ according to eq. 2.27. This figure meets our expectations: crystals have the largest angular velocity around the obstacle at some moderate packing fraction and large activity. As the activity decreases and packing fraction increases the rotation gradually diminishes.

Now if we define a threshold in $\Omega$ as the minimum angular velocity of a crystal to be considered rotating, we can assign a boundary between the region with rotating crystals and non-rotating ones. Crystallization itself of course can only be observed at dense and active enough systems. As the activity decreases, a dramatically larger density of particles is required to form a hexagonal crystal around the obstacle (Fig. 4.25 and 4.26). We summarize all this information in the phase diagram of our system, Fig. 4.27. Here the points on the line that separates the disordered region from the rest are obtained by finding the minimum packing fraction that allows for crystallization around the obstacle for different values of activity. In the disordered phase, no particular crystal forms and the system is more or less homogeneous at different distances to the obstacle. The crystalline phase is not a stationary state either; crystals form, grow, dissolve 

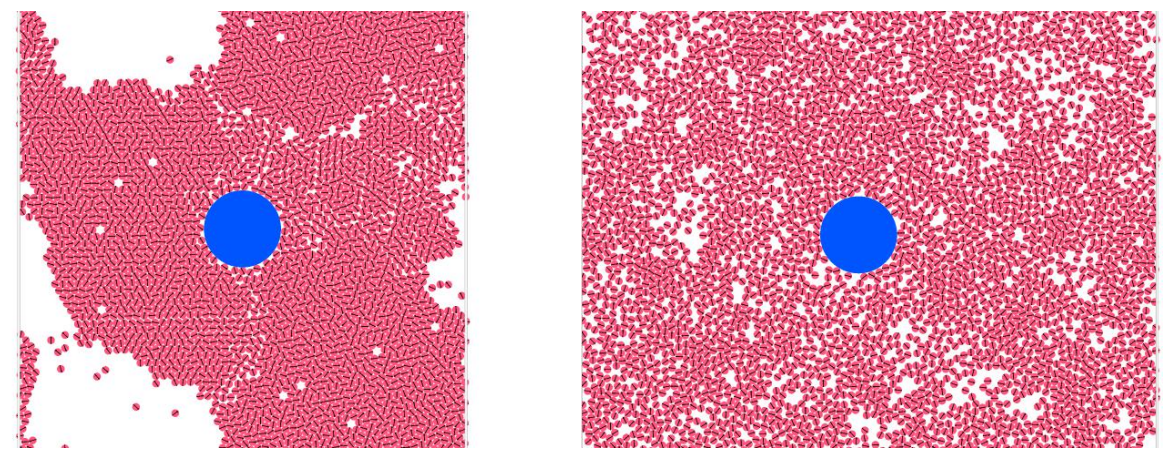

Figure 4.25: Snapshot of a system at Figure 4.26: Snapshot of a system at $\phi_{\mathrm{p}}=0.7$ and $\mathrm{y}=0.0001$, which corresponds to $1-\mathrm{c}=2.5 \times 10^{-5}$. At such high $\phi_{p}$ collective rotations are hampered by $\phi_{p}=0.7$ and $y=0.1$, which corresponds to $1-\mathrm{c}=2.5 \times 10^{-2}$. No other clusters. crystalline cluster forms around the obstacle.

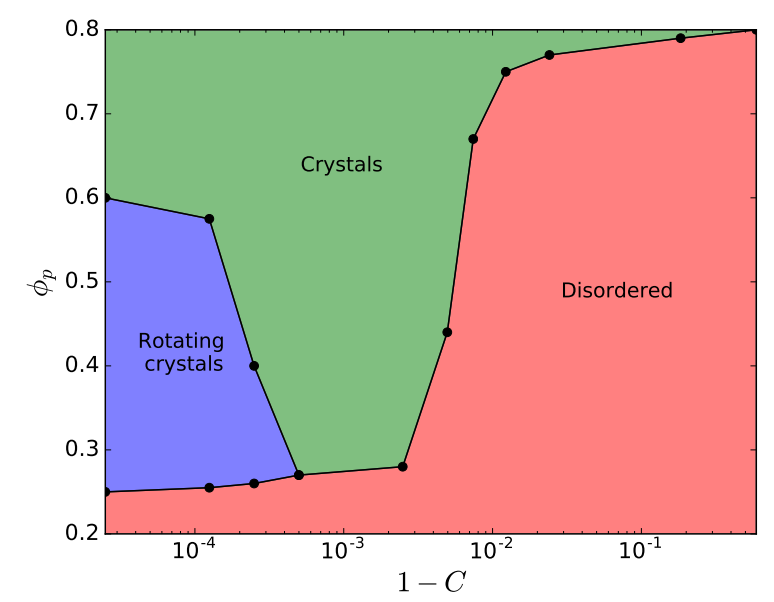

Figure 4.27: Demonstration of possible phases at different values of activity and packing fraction. Explanation about the boundaries between different phases can be found in the main text.

and re-grow around the obstacle all the time. There can be moving crystals far from the obstacle at this region, yet the crystals around the obstacle do not rotate. We take $\Omega^{*}=9 \times 10^{-4}$ against which the phase diagram is relatively robust. 

Motion of self propelling creatures in natural habitats like organelles within the cell, bacteria in soils, microorganisms in blood flow, or fish in the ocean are some examples of the interaction of active particles with crowded environments. Understanding the transport of such particles in heterogeneous media is therefore essential and yet in its early stages. Although the transport of passive ballistic or Brownian particles in heterogeneous environments has been extensively discussed [107-110], theoretical [111] and experimental [112] studies have just started to shed some light on understanding the transport of active particles in such environments.

In this chapter I present the model we use for implementing the heterogeneity and discuss our findings.

\subsection{ACTIVE LORENTZ MODEL}

For the case of passive particles, it has been shown that simplified model systems such as Lorentz model can already explain the generic features of transport. In this model hard disks (obstacles) are randomly distributed into the system and a point tracer explores the void space between the obstacles. The obstacles are uncorrelated and may overlap in the simplest variant of this model. By increasing the number of obstacles, the void space that is accessible for the tracer reduces more and more until the point where it stops percolating through the system. The tracers at larger densities of obstacles cease to diffuse throughout the system and localize at finite cages surrounded by obstacles. The transition between the diffusing and the localizing phase has a geometric origin, and occurs at a certain density of obstacles $\phi_{c}$. This "percolation transition" of the continuum is accompanied by a series of power-laws. Namely the diffusion coefficient of a passive tracer is known to scale like $\mathrm{D} \sim|\epsilon|^{\mu}$ by approaching the critical density. Here $\epsilon=\left(\phi_{c}-\phi\right) / \phi_{c}$ measures the distance to the critical point. $\mu$, being called the conductivity exponent for historical reasons, is a function of the dimensionality and dynamics of the tracer [109] and acquires the value $\mu=1.310$ for a two-dimensional Lorentz model with passive Brownian tracers [113].

We implement the crowdedness similarly with the Lorentz model and study the motion of active tracers of the same size of obstacles $\left(R_{p}=\right.$ $\mathrm{R}_{\mathrm{o}}$ ) with a small packing fraction $\phi_{\mathrm{p}} \approx 0.001$ (to minimize particleparticle interactions) in such a medium. $N_{p}=100, N_{o}=10000$ in our simulations unless said otherwise. We distribute the obstacles one by 
one and randomly in our simulation systems with the only constraint that they do not overlap. The percolation transition in our variant of the Lorentz model occurs at $\phi_{c}=0.22$ [110] above which the void space does not percolate and is separated into finite isolated clusters.

\subsection{TRANSPORT CHARACTERIZATION}

We begin the analysis of particles' trajectories by plotting their distribution of displacements. In the absence of any obstacles the displacements (say in $x$ direction) of a particle during a time window $\tau$ starting from an initial time $t_{0}, \Delta x(\tau)=x\left(t_{0}+\tau\right)-x\left(t_{0}\right)$, are independent of $t_{0}$ at large $\tau$ and the central limit theorem predicts a Gaussian distribution for them. However, the spatial heterogeneity of the environment renders the motion non-Gaussian through encoding information in higher cumulants of the displacement, so that $\left\langle\Delta x^{2}\right\rangle$ is no longer sufficient for describing the statistics of the displacement. The deviation of $\mathrm{P}(\Delta \mathrm{x})$ from Gaussian by increasing the obstacle density can be observed in Fig. 5.1. The distribution at small to moderate $\phi$ is a Gaussian, with the exception of an extra peak at zero displacements which refers to unlucky tracers trapped in small cages at all times. At large $\phi$ however, $\mathrm{P}(\Delta \mathrm{x})$ deviates considerably from a Gaussian.

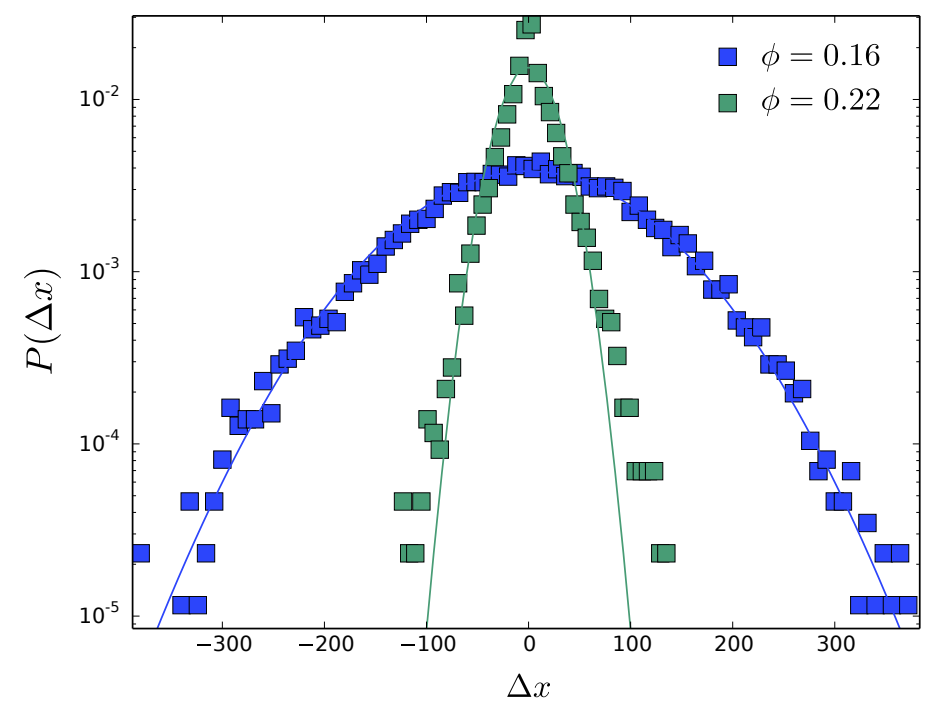

Figure 5.1: Distribution of the displacement of active tracers during a time interval $\tau=10000$ at different density of obstacles. The markers correspond to the simulation data points and the solid lines represent the fitted curves to the data points. Distributions are normalized and the fitted functions are normal distributions with standard deviation of the corresponding data points. 
A proper quantity to illustrate the differences to a Gaussian distribution of displacements is the non-Gaussian parameter defined as

$$
\alpha_{2}(\tau)=\frac{1}{2} \frac{\left\langle\Delta \mathrm{r}^{4}(\tau)\right\rangle}{\left\langle\Delta \mathrm{r}^{2}(\tau)\right\rangle^{2}}-1
$$

Here brackets \langle\rangle denote an average over tracers, a moving time average, as well as an average over different realizations of the heterogeneity. This quantity vanishes for two-dimensional Gaussian transport. Fig. 5.2 illustrates $\alpha_{2}(\tau)$ versus $\tau$. Large time displacements at small obstacle density obey Gaussian statistics and yield a vanishing $\alpha_{2}$ (not shown). At moderate densities, where few particles may be trapped in small cages at all times, this quantity decays to a finite value close to zero, as suggested by Fig. 5.2. The growth of the nonGaussian parameter at intermediate times is due to the presence of obstacles. At the critical point, $\phi=\phi_{c}=0.22$, this quantity diverges for large $\tau$ as predicted by [114].

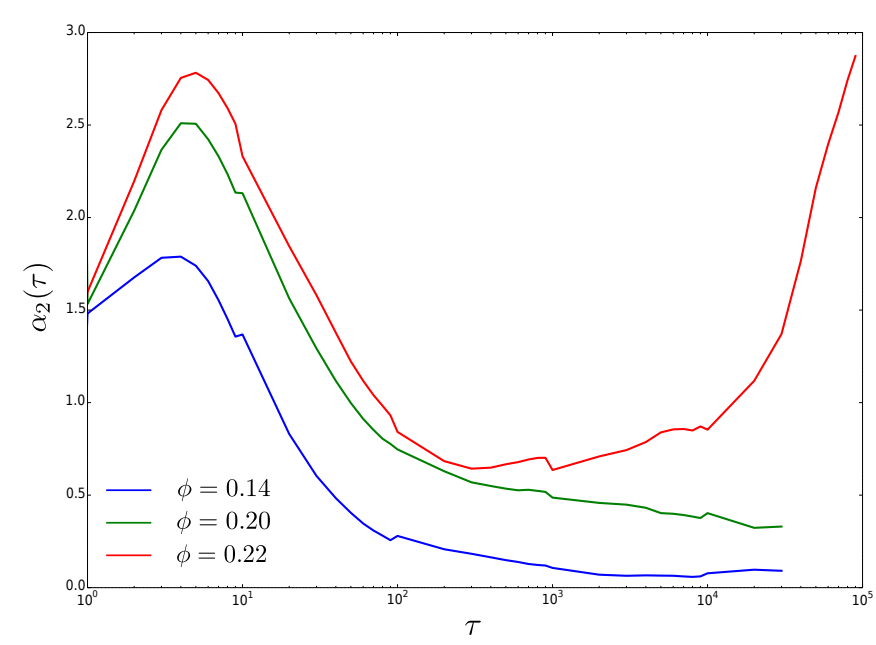

Figure 5.2: The non-Gaussian parameter versus time for different obstacle density. This quantity diverges at the critical point $\phi=0.22$.

Furthermore we plot the mean square displacement (MSD) of active tracers at different obstacle densities in Figure 5.3. At short times the data for all densities collapse on a single line which reflects the ballistic motion before the tracers encounter any obstacles. The shorttime ballistic regime is followed by a crossover to a long-time diffusive regime where the MSD grows linearly with time. At intermediate densities, the presence of obstacles, induces anticorrelations in the velocity autocorrelations which appears in the form of a transient subdiffusive regime at long times. There is an increasingly larger crossover time to the diffusive regime as the critical point is approached, so that at $\phi_{c}$ the subdiffusive regime lasts for the whole window of the simulation time (almost four decades). Increasing the obstacle density 


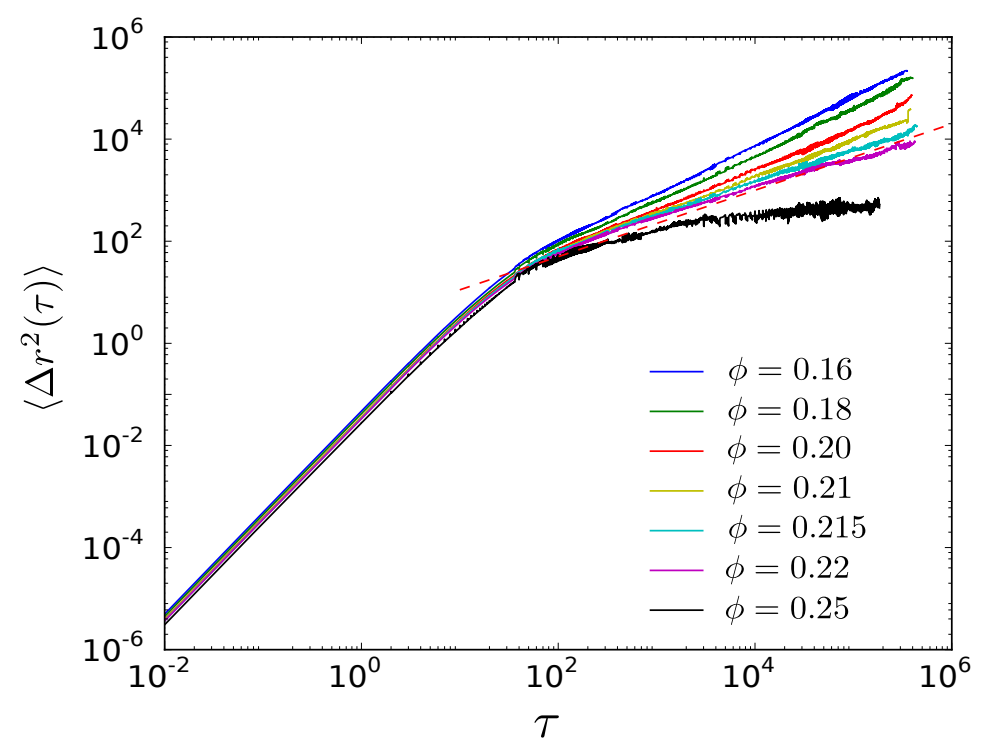

Figure 5.3: Mean square displacement of active tracers $(y=0.1)$ in systems with different obstacle density $\phi$. The minimum system size corresponds to $\phi=0.16$ and is $L=450$. The dashed line $\left(\sim \tau^{0.65}\right)$ is to compare the growth of the MSD at the critical point with ref. [113].

above $\phi_{c}$, the MSD saturates, implying that all particles are localized. Close to the critical point at $\phi_{c}=0.22$ the system is quite dense and the number of collisions is high. This makes our event driven simulations run slower and require increasingly longer times to reach the diffusive regime. Specifically one trajectory for several decades up to the point when it becomes diffusive at $\phi=0.21$ takes about 1 month on a $3.30 \mathrm{GHz}$ Intel Core i3-3220 machine. The extreme slow down of the computational time impedes the calculation of asymptotic values of quantities like the translatoinal diffusion coefficient. Below, we pursue the calculation of some quantities which are accessible in our simulation time window and may provide indirect information about the scaling behavior close to the critical point.

\section{$5 \cdot 3$ SCALING BEHAVIOR}

We are interested in finding out how the tracers' transport properties depend on the distance to the point of the percolation transition ( $\epsilon=$ $\left.\left(\phi_{c}-\phi\right) / \phi_{c}\right)$. Namely how their translational diffusion coefficient $D_{t}$, or the time they require to translate in average by a certain amount, scales with $\epsilon$. The former, as discussed above, is hard to achieve given the slow down of simulations near criticality. The latter however, can be investigated by introducing the quantity

$$
\mathrm{Q}(\tau, \mathrm{d})=\left\langle\theta\left(\mathrm{d}-\left|\mathbf{r}\left(\mathrm{t}_{0}+\tau\right)-\mathbf{r}\left(\mathrm{t}_{0}\right)\right|\right)\right\rangle
$$


Here $\theta$ is the Heaviside function. $Q(\tau, d)$ measures the fraction of particles that have moved less than $d$ during the time interval $\tau$. The average is over particles as well as different $t_{0}$. This quantity is illustrated in Fig. 5.4 for an active system with different packing fractions. For all densities, $\mathrm{Q}(\tau, \mathrm{d})=1$ at small times and gradually vanishes; as long as the continuum percolates, particles can move unboundedly. In assigning a value to $d$ we note the following points: $i$ ) $A$ value of $\mathrm{d}$ smaller than a typical cage size is not helpful since we demand that above the percolation transition no particles translate by larger than d. ii) An extremely large value of $d$ provides us with no helpful information either since $Q(\tau, d)$ would not vanish at large $\tau$ for any $\phi$ then. We have plotted $Q(\tau, d)$ for $d=100 R_{o}$ and $d=50 R_{o}$ for systems with 10000 obstacles and different $\phi$ : see Fig. 5.4 and 5.6.

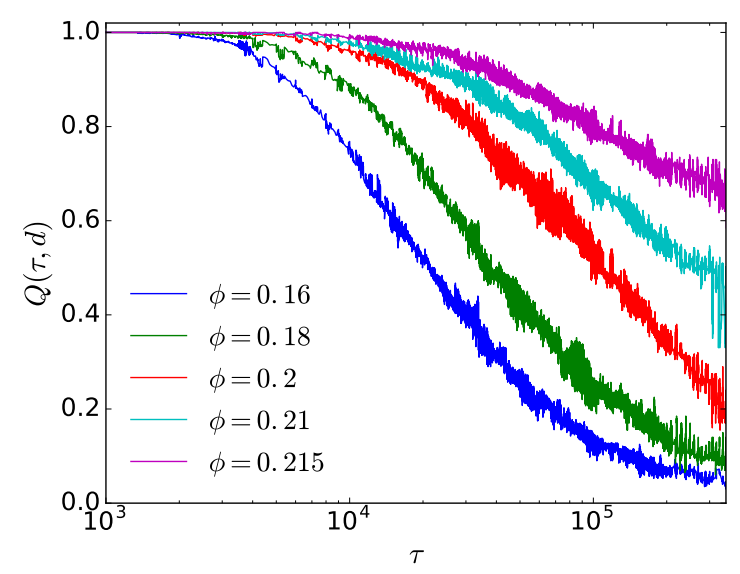

Figure 5.4: Fraction of active particles $(y=0.1)$ that have moved less than $d=100 R_{o}$ in time interval $\tau$, for different packing fractions.

We expect this quantity to be connected to the diffusion coefficient. To investigate this issue, we extract the diffusion coefficients from the MSD plot (Fig. 5.3). Close to the critical point the values of $D_{t}$ we find, are only approximates, found by extrapolating the curves in Fig. 5.3 into the diffusive regime. We rescale the time by these values of $D_{t}$ and observe that almost all the curves collapse on a single one at large times (Fig. 5.5). We are aware of the fact that the values of $D_{t}$ we used for rescaling the time, are only approximates and Fig. 5.5 is just to indicate that the time, $\tau^{*}$, that $Q(\tau, d)$ relaxes to a small value, $\mathrm{Q}^{*}$, and the diffusion coefficient are closely connected. Therefore we investigate the dependence of $\tau^{*}$ to the distance to the critical point and assume that the scaling of $\mathrm{D}_{\mathrm{t}}$ can be found accordingly.

The relaxation time $\tau^{*}$, the time that $Q(\tau, d)$ (for $d=50 R_{o}$ ) drops to $\mathrm{Q}^{*}=0.6$, is obtained from Fig. 5.6 and 5.7 for active and passive systems respectively and illustrated in Fig. 5.8. It appears to us that $\tau^{*}$ grows in the form of a power-law by decreasing $\epsilon$, although the 


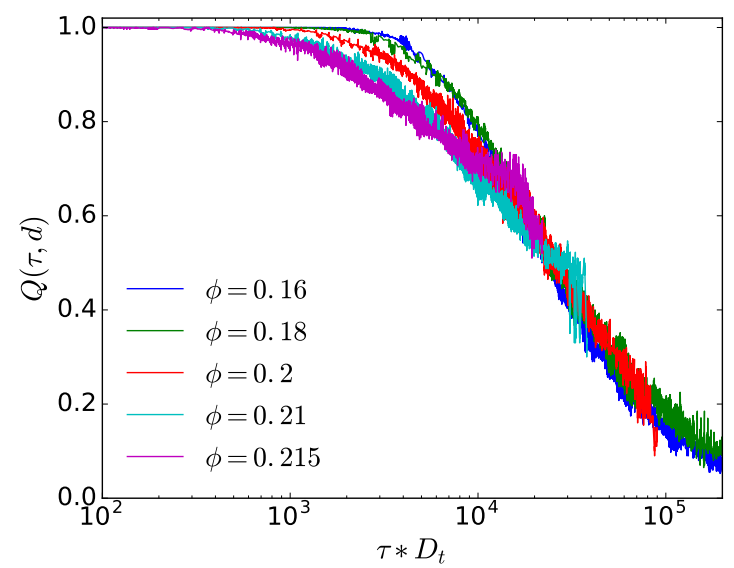

Figure 5.5: Similar curves as the ones in Fig. 5.4, with the x-axis being rescaled by the diffusion coefficient of the corresponding system. All the curves collapse on the top of each other.

statistics require further improvement for a better judgment. This is not surprising, noting the previous works on the dynamics of passive tracers in the Lorentz model with overlapping obstacles where $D_{t} \sim$ $\epsilon^{1.310}[113]$.

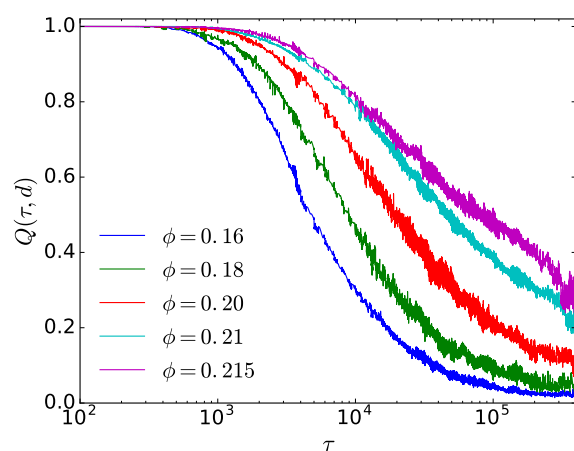

Figure 5.6: Fraction of particle that have moved less than $\mathrm{d}=50 \mathrm{R}_{\mathrm{o}}$ in active systems $(y=0.1)$ with different obstacle density.

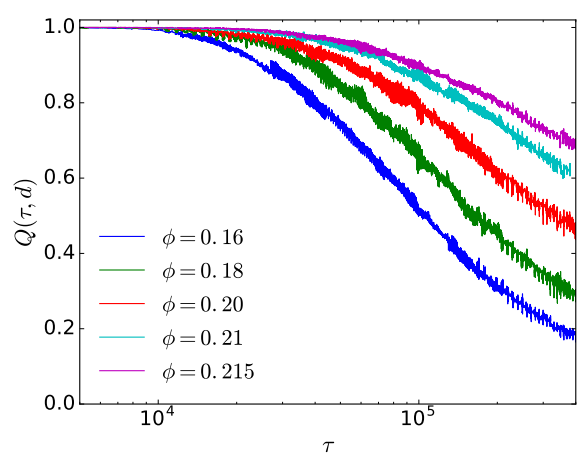

Figure 5.7: Fraction of particle that have moved less than $\mathrm{d}=50 \mathrm{R}_{\mathrm{o}}$ in passive systems $(y=10)$ with different obstacle density.

We have tried to carry out finite size scaling at this point, the result of which is summarized in appendix A.

\subsection{EXPLORATION OF CAGES}

We have learned so far that above the percolation transition $\left(\phi>\phi_{c}\right)$ tracers are trapped in finite cages surrounded by obstacles. The mean 


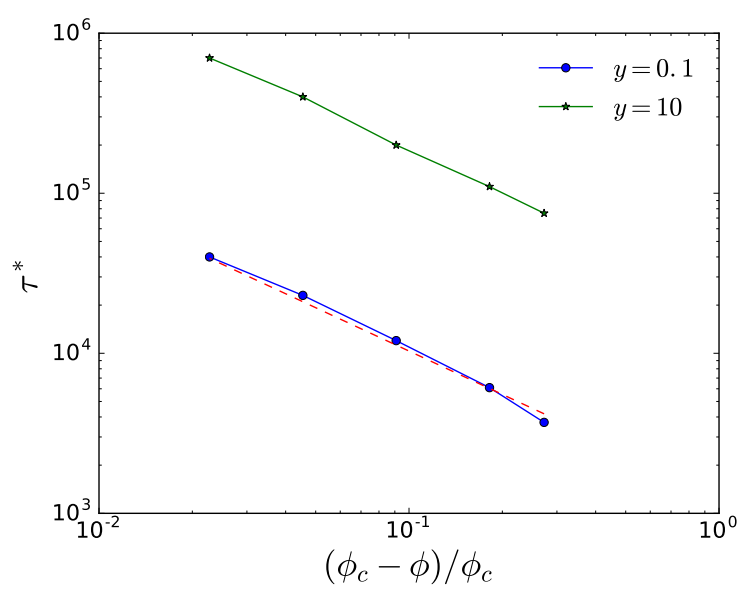

Figure 5.8: $\tau^{*}$, the time that $Q(\tau, d)$ (given in Fig. 5.6 and 5.7) drops to o.6, as a function of the distance to the critical point for an active and passive system. $\tau^{*}$ appears to diverge in the form of a power-law at the critical density for both passive and active systems. The dashed line corresponds to $\tau^{*} \sim \epsilon^{-0.9}$.

square displacement of particles in this case is limited by the size of such cages and converges to a constant value at large times. At intermediate times, activity gives rise to the enhancement of the MSD: Fig. 5.9 suggests that increasing $y$ from $y=0.1$ to $y=10$ increases the MSD by about one order of magnitude at around $100<\tau<1000$. The saturation value of the MSD at large times is however independent of the dynamics and is only a function of cage sizes. A question of interest, which I will pursue below, is where and how activity plays an important role with respect to the motion of these caged particles?

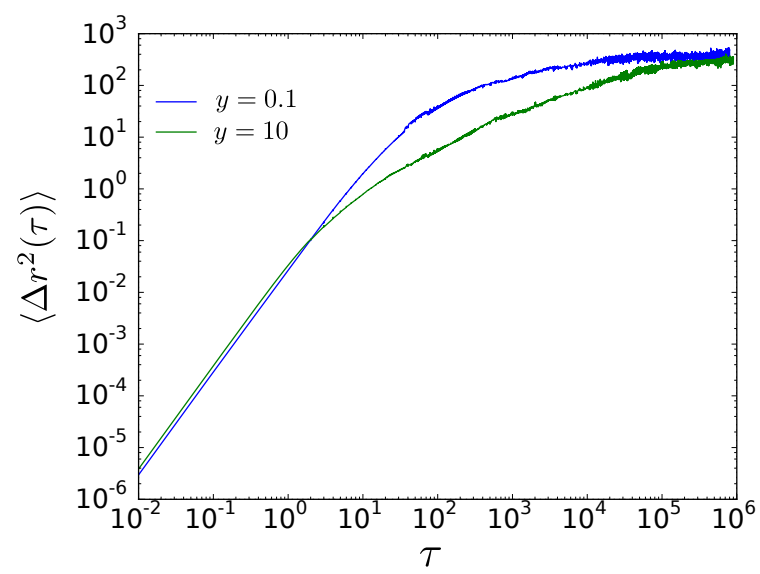

Figure 5.9: The mean square displacement of tracers with different activities in obstructed systems above the percolation transition $(\phi=0.26)$.

Observing the trajectory of particles (Fig. 5.10), active tracers seem to move along the inner wall of the cages for a long distance com- 
paring to passive tracers, before they change their direction. This is no surprising observation as we have already discussed the motion of a single particle around an obstacle in chapter 3 and have learned active particles tend to stick to the walls.

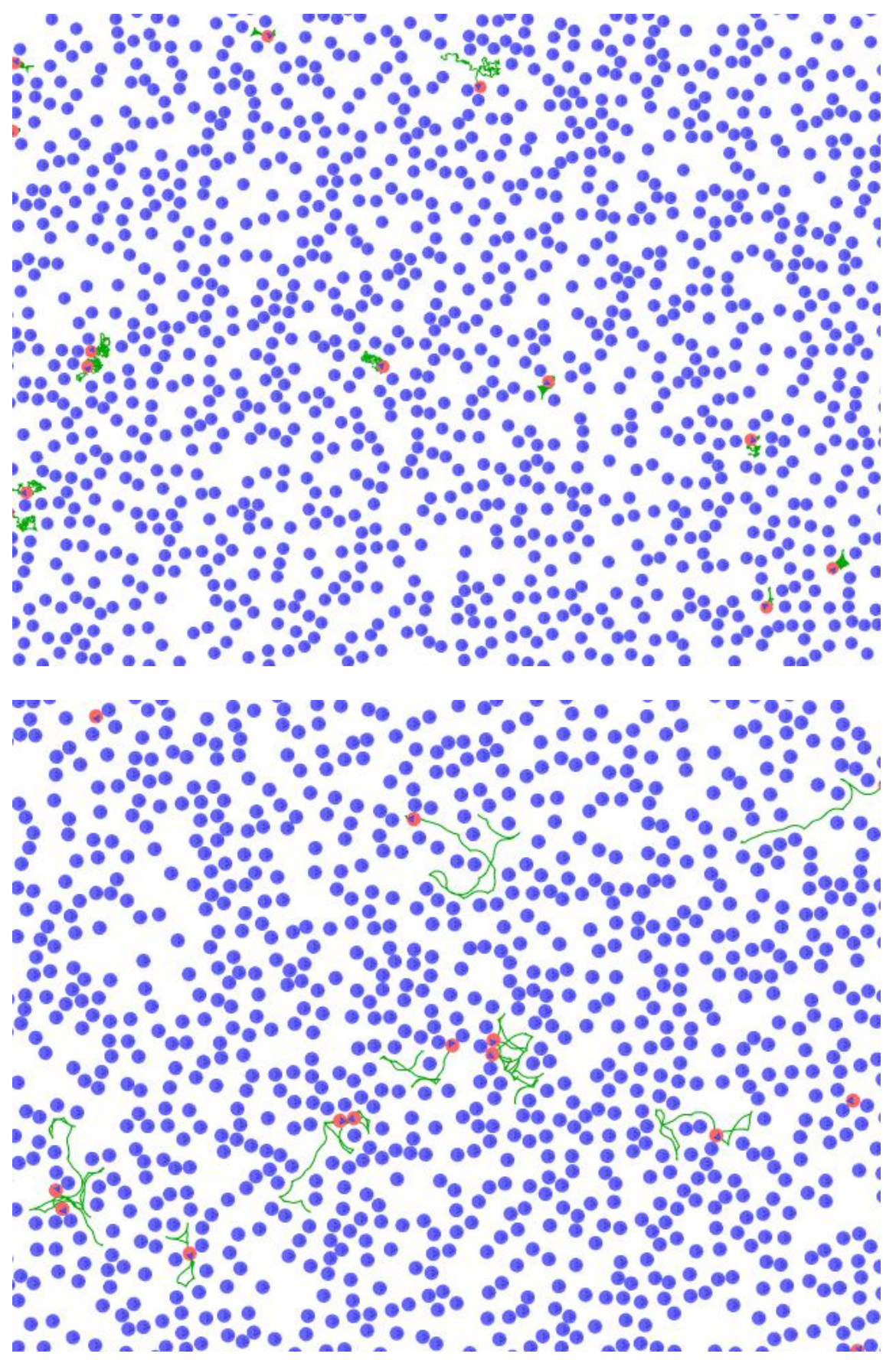

Figure 5.10: Trajectory of particles for $\Delta t \sim 300$ in systems with $\phi=0.26$ and $y=10$ (top), $y=0.1$ (bottom). Passive particles' trajectories are rather coiled as compared to active particles' extended trajectories which pass by several obstacles before re-orienting. 
We try to pinpoint this distinction between active and passive systems by comparing the distribution of square displacements at some time interval $t=500$. Distribution of the squared displacement of all particles during time $t$ starting from different initial times is plotted in Fig. 5.11.

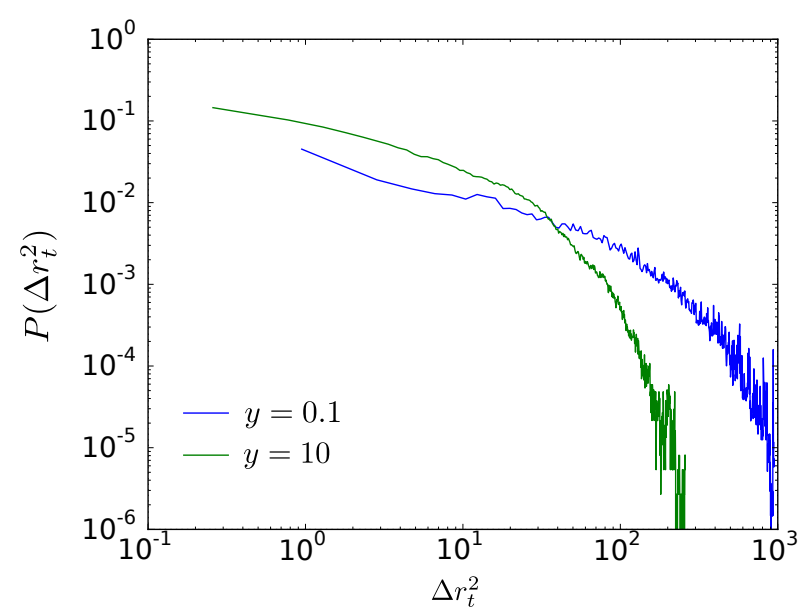

Figure 5.11: Comparison of the distribution of square displacements at a time interval $t=500$ above the percolation threshold in two active and passive systems: $\phi=0.26, \mathrm{~L}=350$.

From Fig. 5.9 it is expected to see larger displacements at this interval in active systems. When we rescale the two distributions by their mean value, they collapse on a single curve: Fig. 5.12. This implies that the only important quantity regarding the displacements of particles is the MSD.

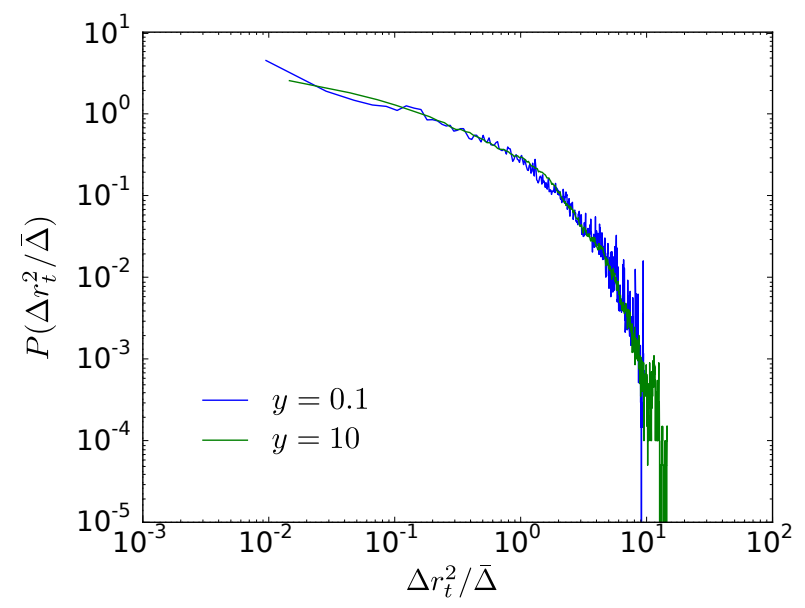

Figure 5.12: Distribution of square displacements of particles divided by their mean square displacement $(\bar{\Delta})$ in two active and passive systems. 
In order to compare the extension of trajectories of passive and active tracers, we compute the radius of gyration, $R_{G}$, of their trajectories in the two systems. We consider the trajectories of our random walkers as long polymers, where each monomer $i$ is located at $\mathbf{r}_{t_{i}}$. The square radius of gyration is then

$$
R_{G}^{2}(\tau)=\left\langle\frac{1}{2 N^{2}} \Sigma_{i=1}^{N} \Sigma_{j=1}^{N}\left(r_{t_{0}+t_{i}}-r_{t_{0}+t_{j}}\right)^{2}\right\rangle,
$$

where $\tau=t_{N}$ and the average is over the tracer particles, different obstacle configurations, and $t_{0}$. The result is plotted in Fig. 5.13 for active and passive systems. $R_{g}(\tau)$ vanishes at small $\tau$ and grows faster for active particles. It does not appear, at least in our window of simulation time, that the two curves corresponding to two activities will converge onto a single curve at large $\tau$. We conclude then that the trajectories of active tracers in cages are more extended than the passive ones, which are rather coiled. This is in agreement with the preference of active particles to stick to and move along the walls, discussed in chapter 3 .

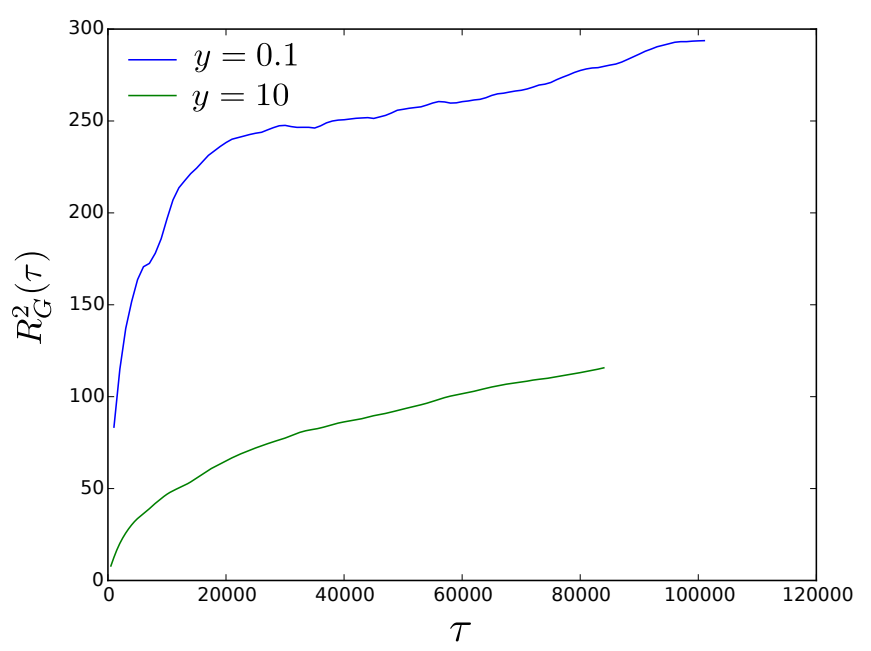

Figure 5.13: Squared radius of gyration of the trajectories of tracer particles during a time interval $\tau$ for systems with $\phi=0.26$ and $L=350$.

We proceed with investigating the role of tracer's activity in their exploration of cages in slightly modified systems. We demand our particles to be smaller than the obstacles to make our results in this section comparable to our results in previous chapters. The percolation transition in our model with $R_{p} / R_{o}<1$ is studied in [115]: for an obstacle density of $\phi_{\mathrm{o}}=0.4$ the maximum particle radius that can diffuse throughout the whole void space is $R_{p} / R_{o}=0.4$. From here on, we investigate the systems with this density and size of obstacles. Fig. 5.14 demonstrates the trajectories of some active particles in such a medium. 


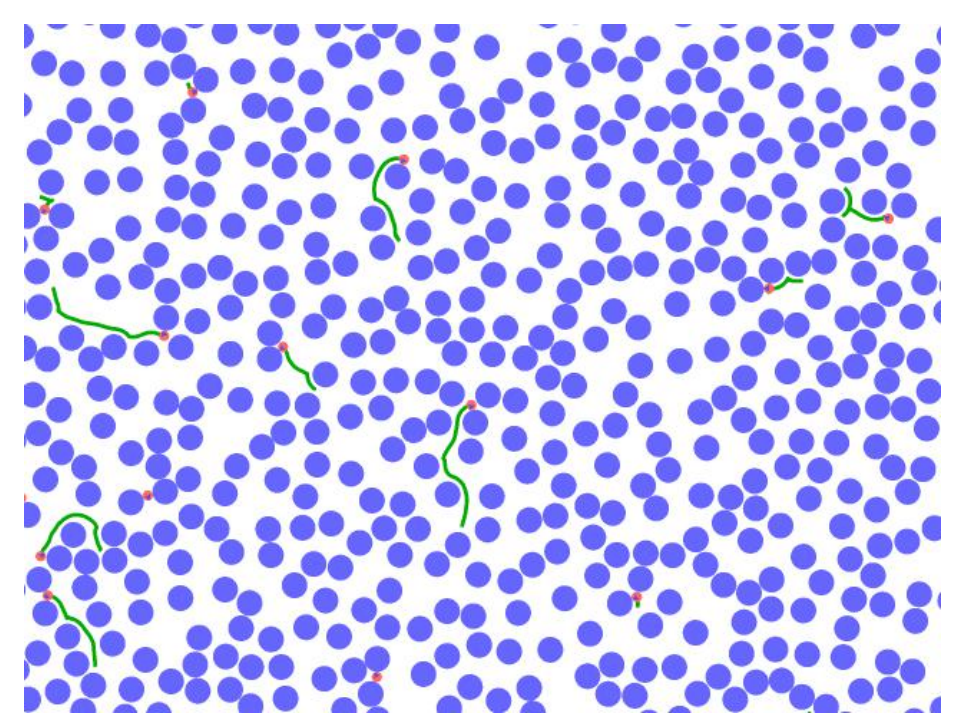

Figure 5.14: Sample trajectories of particles in a system with $y=0.1, \phi=$ $0.4, N_{p} / N_{o}=100 / 5000, R_{p} / R_{o}=0.4$. For a particle to obstacle size ratio of $R_{p} / R_{o}=0.4$, the critical density is about $\phi=0.4$.

Now we introduce the pair correlation function of obstacles and particles, $g(r)$, to further improve our understanding of the differences between trajectories of active and passive particles in this regime. This quantity is plotted in Fig. 5.15. Height of the first peak according to the definition can be found from

$$
g\left(R_{p}+R_{o}\right)=\frac{M}{N_{p}} \frac{L^{2}}{2 \pi \Delta\left(R_{p}+R_{o}\right) N_{o}},
$$

where $\frac{M}{N_{p}}$ is the fraction of particles that are immediately next to an obstacle. For our set of parameters that is indicated in Fig. 5.15 one expects to see a very small first peak in passive systems, where most of particle are deep in the bulk (in the middle of cages) or equivalently $M / N_{p} \approx 0$. On the other hand our active particles move along obstacles for a long time and $M / N_{p} \approx 1$ results in the height of the first peak to be about 18. This value in Fig. 5.15 is even larger because some of the particles are immediately next to more than one obstacle at the same time and are counted more than ones.

Equivalently the number of collisions with obstacles reveals how close particles move relative to obstacles. This quantity also increases dramatically with activity (Fig. 5.16).

In order to demonstrate the motion of active particles close to the inner wall of cages we can explicitly calculate the distance of particles to their closest obstacle, $d$. I define $d$ for each particle at an instance of time as the shortest of the two distances that the particle has from the obstacle that it hit previously and the obstacle it will hit next. This is not necessarily the closest obstacle, but it captures the behavior that we are interested in, and since we use an event-driven algorithm this 


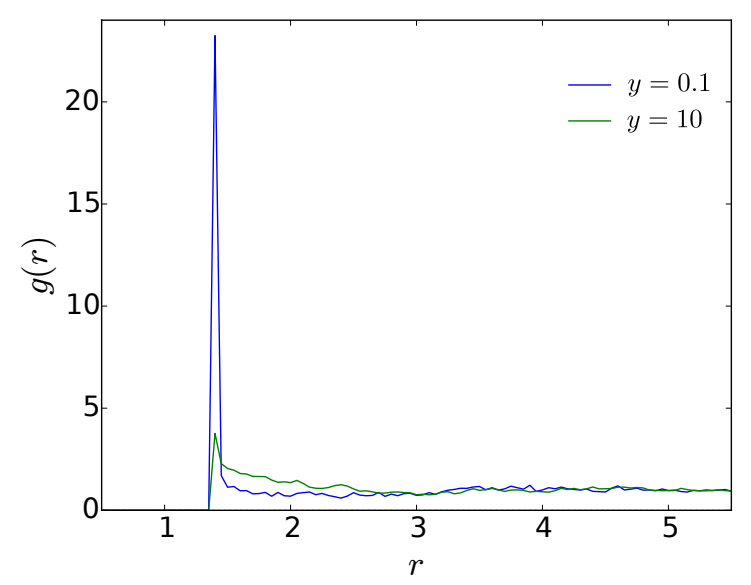

Figure 5.15: Obstacle-particle pair correlation function in systems with different activities above the percolation transition. $\phi=$ $0.4, N_{p} / N_{o}=100 / 5000, R_{p} / R_{o}=0.4, L=198.166$. Active particles rather move in the vicinity of the obstacles, whereas passive ones are indifferent about them.

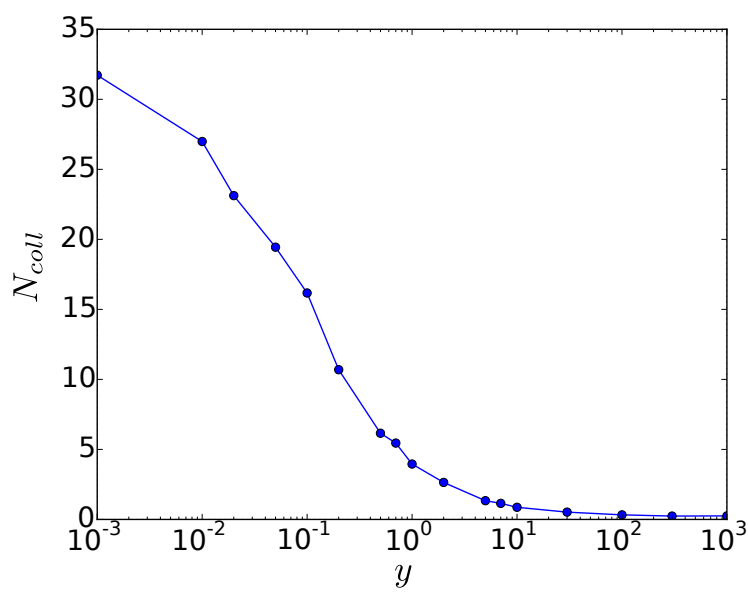

Figure 5.16: Number of collisions with obstacles in systems above the percolation transition versus $y$. This number is per particle and during one unit of time. Each marker corresponds to a system similar to Fig. 5.15 with different $y$.

quantity is easy to calculate. Fig. 5.17 shows this quantity for similar geometries but different activities, just after the simulation starts, before the system reaches the steady state. In this case $d$ is independent of activity and depends only on the initial configuration. As the simulations run longer, the difference between active and passive particles become apparent: Active particles maintain a very small distance to obstacles (Fig. 5.18). 


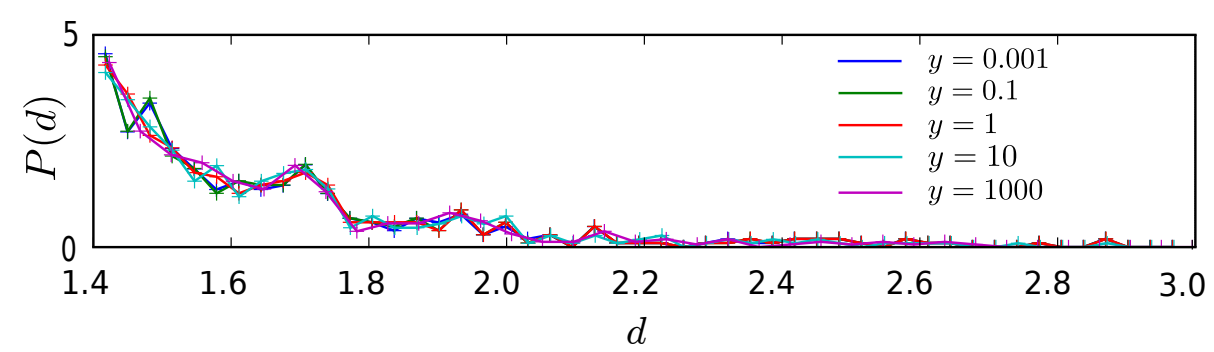

Figure 5.17: Distribution of the distance to the closest obstacle, in a time window before reaching the steady state. $\mathrm{N}_{\mathrm{p}} / \mathrm{N}_{\mathrm{o}}=$ $100 / 5000, R_{p} / R_{o}=0.4, \phi=0.4, L=198.166$.

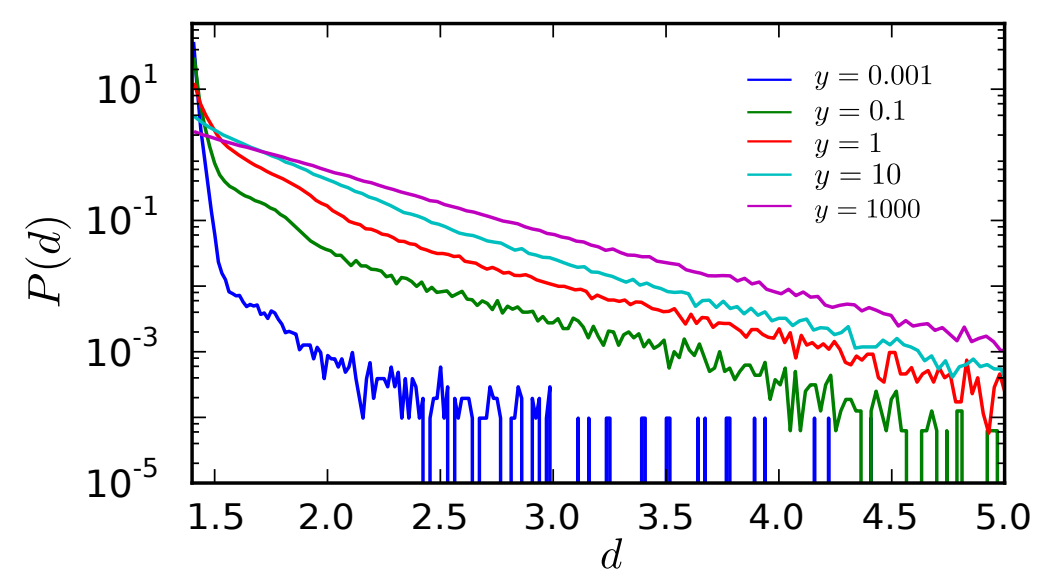

Figure 5.18: Distribution of the distance to the closest obstacle, in a time window in the steady state. System parameters are similar to Fig. 5.17.

The average of $d$ is plotted as a function of $y$ in Fig. 5.19. This quantity clearly reveals the role of activity in the exploration of cages: Highly active tracers move along the walls of the cages, while being almost stuck to it ( $\overline{\mathrm{d}} \approx \mathrm{R}_{\mathrm{p}}+\mathrm{R}_{\mathrm{o}}$ ). Whereas passive ones swim in average somewhere in the middle of the cages ( $\overline{\mathrm{d}}$ for them is a function of the average cage size only). 


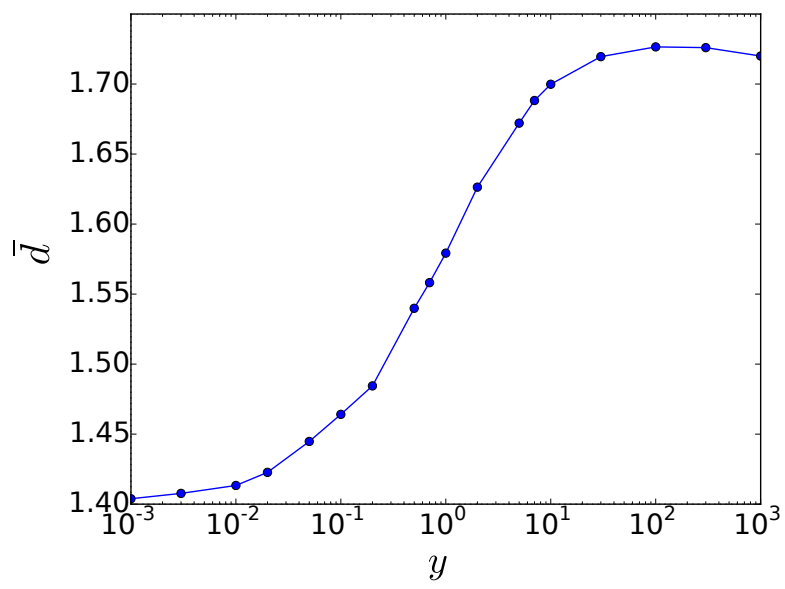

Figure 5.19: Average distance of particles to the closest obstacle, versus $y$ in systems above the percolation threshold. This quantity reveals a crossover from moving almost tangent to the obstacles in active systems, to wandering in the middle of the cages in passive ones. 


\subsection{SUMMARY}

The motion of self-propelled particles in homogeneous environments, isolated from any other object, is an exception rather than a rule. Biological active particles, whether microorganisms in human organs/blood/soil/etc., or fish in the ocean, experience frequent interactions with the walls and obstacles. Unlike systems in equilibrium, the heterogeneity in active systems can do more than just confining the particles (like walls in passive systems) or slowing down of their motion (like steric interactions of passive particles with many immobile obstacles). Phenomena like circular motion of bacteria close to the container planar walls [13], accumulation of sperm cells on solid surfaces [19], formation of biofilms [95], and enhancement of Trypanosome's propagation in crowded media [23], are some of the examples that reveal the surprising interplay of heterogeneity and activity. Despite the ubiquity in nature, a major part of the studies on active particle's behavior has been devoted to homogeneous systems. In order to make a contribution to the understanding of active particle's respond to environmental heterogeneities, in this work we have employed a minimal model for active particles to investigate their individual dynamics and the emergent collective behavior in the presence of obstacles.

We have found out that the residence time of a particle on a flat wall depends not only on its incident angle and angular velocity, but also on its activity: The more active, the longer the particle resides on the wall. The average residence time increases in the form of a power-law with activity in active systems, diverging at zero rotational diffusion. Interaction of active particles with spherical obstacles is found to be qualitatively similar, with the two main differences: The average residence time no longer diverges at large activities, it is rather bounded by the finite size of the obstacle. Also raising the magnitude of the active force decreases the average residence time on such obstacles, unlike the case of a planar wall.

We have shown that at moderately dense and highly active systems, the crystalline cluster formed around the obstacle performs systematic spontaneous rotations. The rotations can be understood based on a simple argument to model collisions with moving rough walls, mimicking the periphery of the rotating crystal. The feedback mechanism producing the rotations, distinguishes our rotating crystals from 
the previously observed rotating aggregates of active particles: In our system, the onset is due to spontaneous fluctuations of the angular velocity of the whole crystal which get enhanced through the selective attachment of particles to the crystal with respect to their orientation and equivalently through a total torque that scales linearly with the number of particles in the crystal.

Regarding the dynamics of active particles in a crowded medium, we have illustrated that by increasing the obstacle packing fraction towards the percolation transition, the distribution of particle's displacements deviates from the Gaussian, the mean square displacement saturates to a value specified by the average cage size, and the relaxation time (the time a particle requires for moving a considerable length) diverges. Increasing the activity is found to enhance the transport (the translational diffusion constant) below the critical packing fraction. Above the critical point, activity induces extended trajectories along the cage's interior wall which results in a larger radius of gyration and faster transport at intermediate times.

\subsection{OUTLOOK}

The projects that I have covered in this thesis have various avenues for further exploration. Below, I introduce some of these side projects that we have started but there is still work to be done analyzing/understanding our results.

Motivated by the motion of Chlamydomonas and other puller microswimmers, a simple reversing of the normal component of the velocity and preserving the orientation in collisions with surfaces, may not be realistic in some cases. The flow fields generated by a puller microswimmer in the vicinity of a flat wall gives rise to the reorientation of the particle perpendicular to the wall upon hitting it. As a result, the cell leaves the surface immediately after the collision, unlike pusher particles which gradually reorient parallel with the wall and move along it for long times [97].

Inspired by [49] we consider the following scenario for the reorientation of the particles upon collisions with obstacles: The outgoing angle is derived from an empirical distribution with a peak at an angle between 0 and $\pi / 2$. The velocity is accordingly updated after each collision to lie along the new orientation. Such a collision rule may give rise to intrinsically different observations: Does the accumulation around the obstacles survive? Can we define an effective long range repulsive force between the obstacles and particles in contrary to the effective attraction of particles to the obstacles in the previous case? There are many other open questions in this regard that may be worth exploring. 
We have considered hard-core collisions between the particles all over this thesis. However, natural or synthetic active particles may experience short range attractive inter-particle forces as well. This can be e.g. through hydrodynamic interactions [48], depletion potential due to the presence of non-adsorbing polymers in the solvent [8o], or diffusiophoretic interactions [73]. Concerning the clustering phenomenon in active systems, attractive forces are expected to enhance this effect. In order to investigate the phase diagram of an active system in the presence of attraction, we have substituted the repulsive inter-particle potential with a Lennard-Jones (as a side project together with Marko Detmers). In the absence of any obstacles, the system exhibits clustering at intermediate densities. The small clusters scattered all over the system, are not stationary and perform no similar rotations as the ones reported in chapter 4 . They only coarsen into large ones at large densities or attractive strength, where the system quickly becomes frozen: The potential energy dominates the kinetic energy. We have learned in chapter 4 that the effective particleobstacle attraction is a key element in the formation of the rotating crystals and now we know that an explicit inter-particle attraction apparently fails to induce similar systematic rotations in the presence of no obstacles. There are of course many other interesting issues in this regard which require further investigations.

Obstacles in natural habitats are not necessarily immobile. Think of the crowded array of red blood cells, through which a parasite needs to find its way escaping from a white blood cell, or the dead or flagella-less bacteria, with which a mobile bacterium in a biofilm successively encounters. There has been a number of interesting studies on the mixture of active particles and heavy passive particles [116] or polymers [117, 118]. The effect of the active bath on the transport of the passive particles or the swelling of the passive chain is not trivial and would be interesting to further explore.

Throughout this thesis the particles have been assumed to be spherical. This is only a minimal model and usually different from real systems. As a new direction for our studies we have simulated active flexible polymers, representing active elongated particles such as Cyanobacteria, in a homogeneous medium. We have implemented such a polymer as a chain of passive particles interconnected through a harmonic potential and subjected to thermal noise, with the first particle in the chain being active following Eq. 2.9a and 2.9b. We have observed temporarily shaped polar bundles of the particles (Fig. 6.1), which is a common behavior in elongated active systems. Other than the abundant biological examples, the new techniques and progress in chaining the artificial colloids [119, 120] has also opened the possibility of realizing active chains artificially. We would like to explore 
the behavior of such systems under the variation of activity, density, flexibility, and environmental geometries.

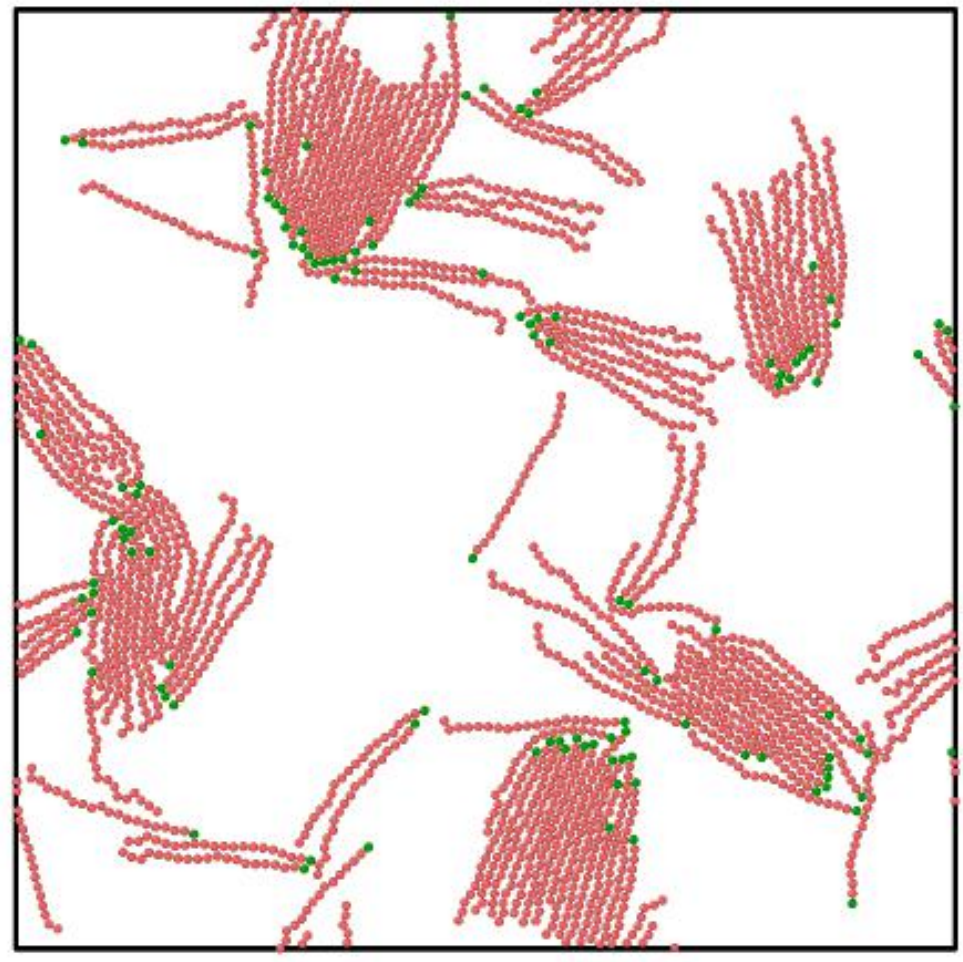

Figure 6.1: A snapshot of our simulation results for active polymers. The red and green beads are the passive and active monomers respectively. Two colliding chains tend to parallelize and gradually form large bundles of aligned chains upon colliding with other chains. The bundles eventually dissolve due to the collisions with other bundles or the rotational diffusion of the active heads, and form again later. 


\section{A.I FINITE SIZE SCALING}

Regarding the percolation transition introduced in chapter 5 , in this section I explain our attempts to do finite size scaling in order to find out how our findings in section 5.3 depend on the system size. The results in this section require further improvements, but we did not proceed with that.

Before carrying out the analysis, I introduce another power-law behavior in the Lorentz model, which is the scaling of the correlation length $\xi$ with the distance to the critical point: $\xi \sim\left|\phi-\phi_{c}\right|^{-v}$. The correlation length is a measure of the size of the largest finite cluster of the void space. Hence it is expected to have a finite value above the critical point where the void space is separated into several finite pieces and increase by approaching the critical point (decreasing $\phi$ toward $\left.\phi_{\mathrm{c}}\right)$. With respect to static properties, continuum percolation in the Lorentz model shares the same universality class as lattice percolation for which the exact value of $v$ in two dimensions is known: $v=4 / 3[107,108]$.

Now in very small systems $(L \ll \xi)$ the correlation length, $\xi$, becomes unimportant, the motion will be limited only by the size of the system, and the main length scale will be given by the box size. In very large systems $(L \gg \xi)$ on the other hand, size of the box will be the irrelevant length scale. Therefore observables in general will be a function of both $\epsilon$ and L. The dynamic scaling ansatz for the relaxation time reads [108]

$\tau^{*}(\epsilon, \mathrm{L})=\xi^{\mu / \nu} \tilde{\tau}^{*}(\mathrm{~L} / \xi) \sim \begin{cases}L^{\mu / \nu} & \text { for } \mathrm{k} \ll \xi \\ |\epsilon|^{-\mu} & \text { for } \mathrm{\gg} \gg \xi\end{cases}$

Fig. A.1demonstrates $\tau^{*} \epsilon^{\mu}$ as a function of $L / \epsilon^{-v}$ for different values of $\mu$ and $v$. Fig. A.2 demonstrates the same quantity with more resolution around $\mu=0.5, v=1.5$ which seem to obey the above equation. Looking into Fig. A.2 we infer that the subplot with $v \approx$ 1.25 and $\mu \approx 0.75$ shows the closest behavior to the above equation: $\tau^{*} \epsilon^{\mu}$ saturates at large $L$ (or $\tau^{*}$ scales as $\epsilon^{-\mu}$ when $L / \epsilon^{-\nu} \gg 1$ ) and follows a power-law with respect to $L / \epsilon^{-v}$ at small $L$ (or $\tau^{*}$ scales as $\mathrm{L}^{\mu / v}$ when $\left.\mathrm{L} / \epsilon^{-v} \ll 1\right)$. 

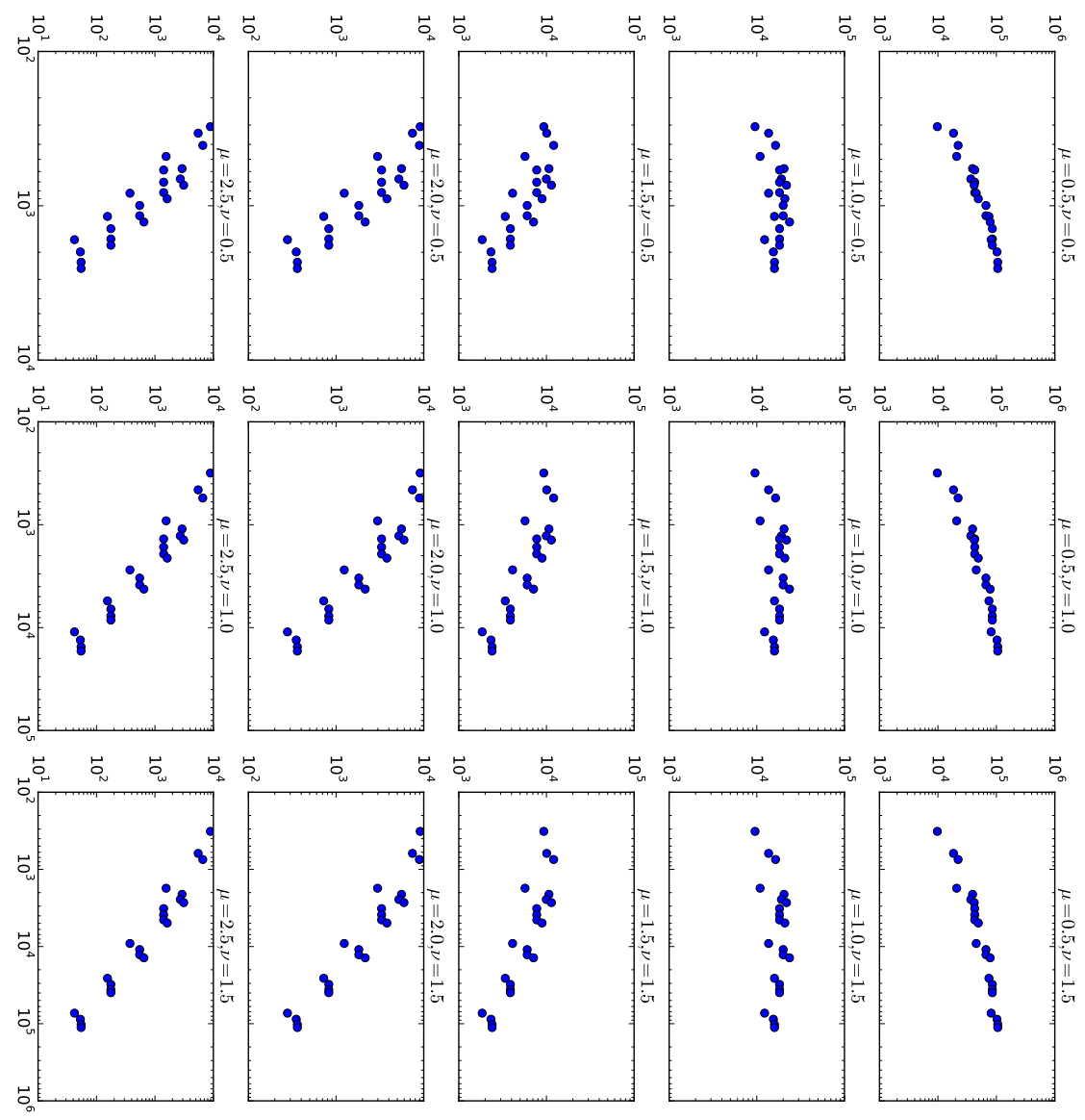

$b_{w}$
$\sigma_{\mathrm{t}}$

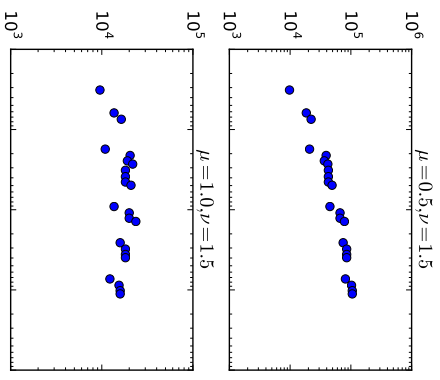

:

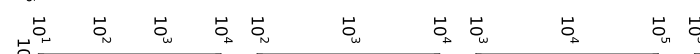

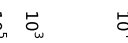
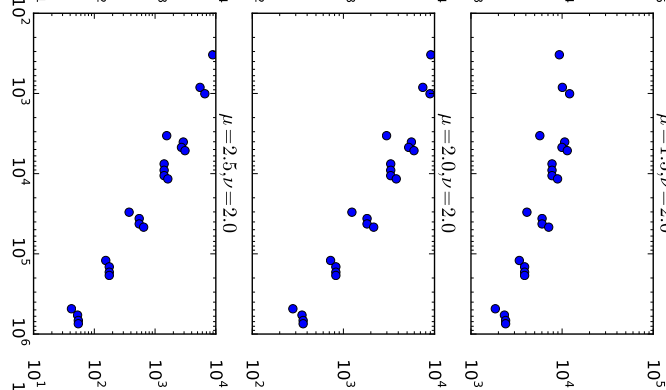

$\vec{\sigma}_{u} \vec{o}_{w} \vec{o}_{+} \vec{o}_{0}$

$\frac{\sqrt{2}}{3}$
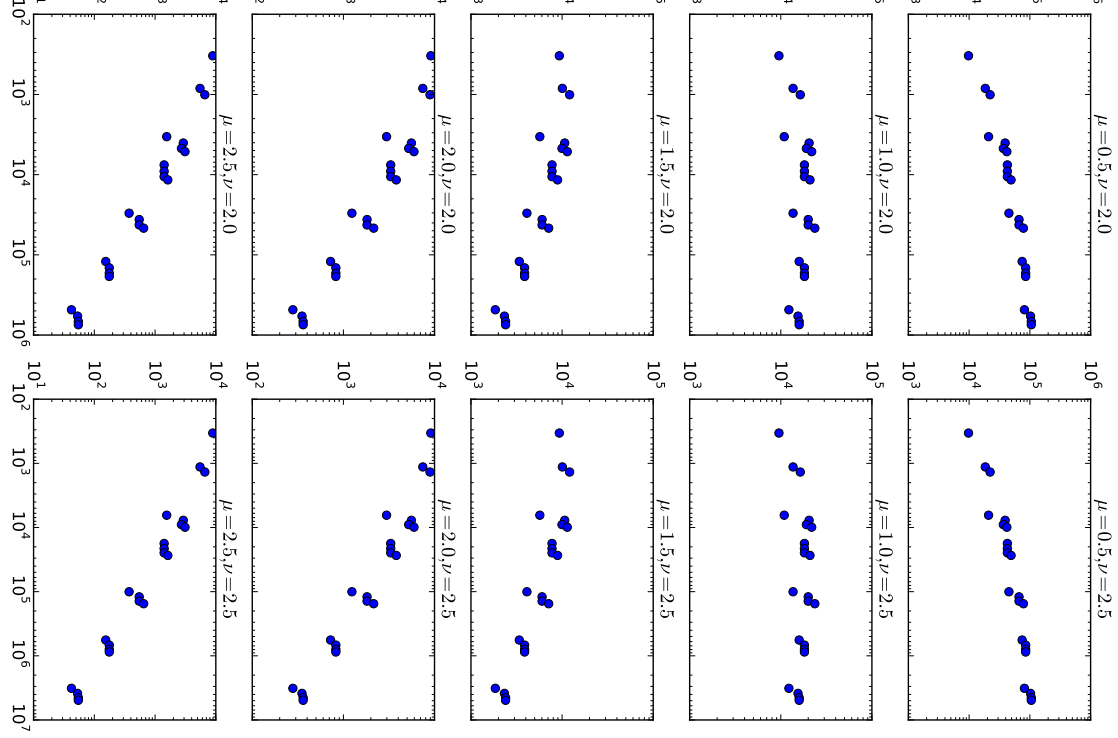

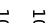

$\vec{\sigma}_{u} \vec{o}_{w} \vec{o}_{i} \quad \sigma_{0}$
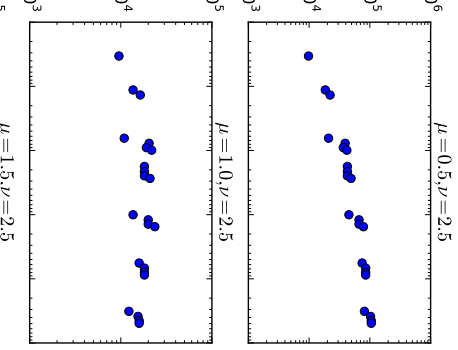


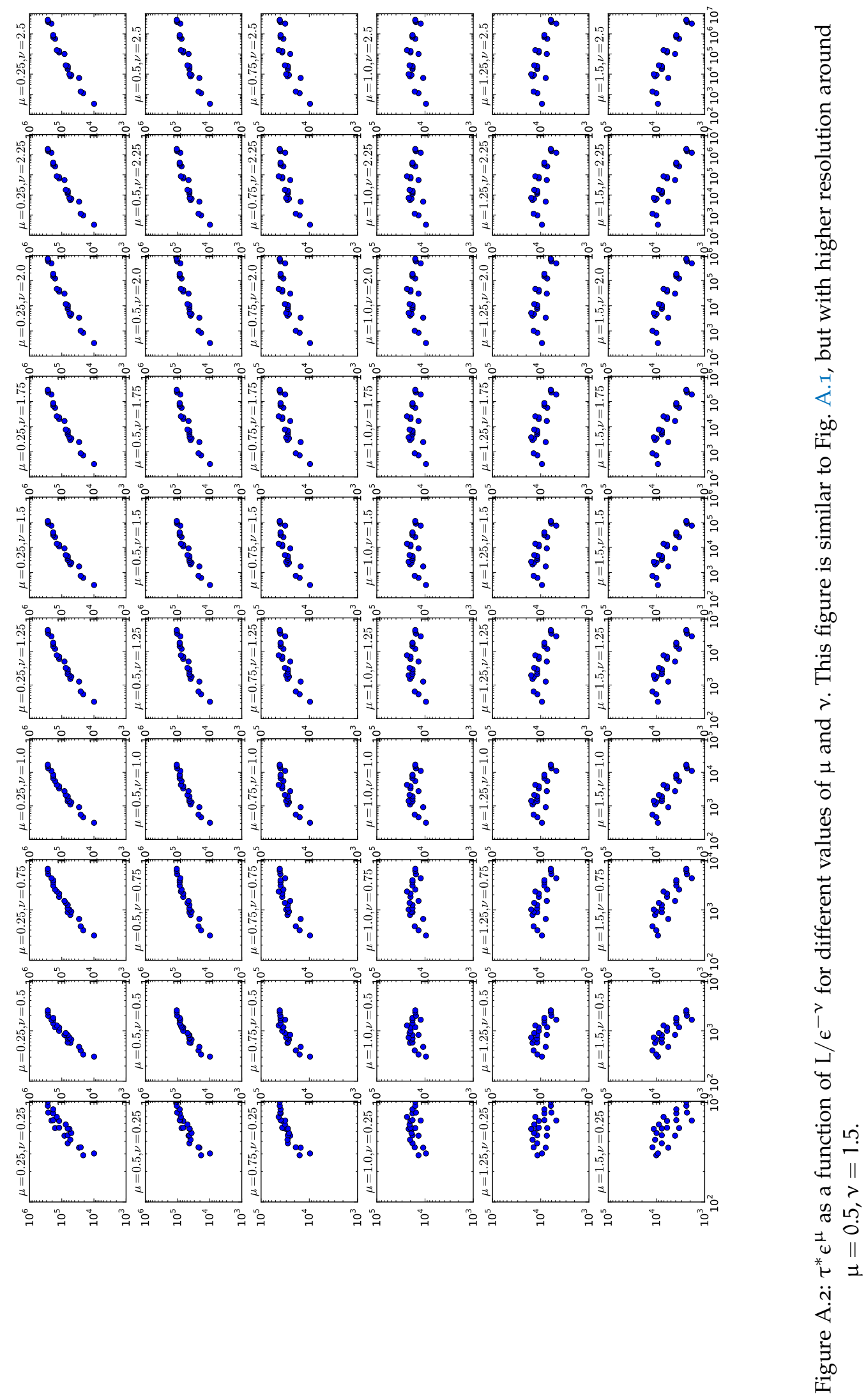



[1] Zahra Mokhtari, Timo Aspelmeier, and Annette Zippelius. "Collective rotations of active particles interacting with obstacles." In: EPL (Europhysics Letters) 120.1 (2017), p. 14001. URL: http: //stacks . iop. org/0295-5075/120/i=1/a=14001.

[2] Reinhold Fürth. "Die brownsche bewegung bei berücksichtigung einer persistenz der bewegungsrichtung. mit anwendungen auf die bewegung lebender infusorien." In: Zeitschrift für Physik 2.3 (1920), pp. 244-256.

[3] Howard C Berg. Random walks in biology. Princeton University Press, 1993.

[4] Howard C Berg. "Bacterial behaviour." In: Nature 254.5499 (1975), p. 389 .

[5] Edward M Purcell. "Life at low Reynolds number." In: American journal of physics 45.1 (1977), pp. 3-11.

[6] Craig W Reynolds. "Flocks, herds and schools: A distributed behavioral model." In: ACM SIGGRAPH computer graphics. Vol. 21. 4. ACM. 1987, pp. 25-34.

[7] Tamás Vicsek, András Czirók, Eshel Ben-Jacob, Inon Cohen, and Ofer Shochet. "Novel type of phase transition in a system of self-driven particles." In: Physical review letters 75.6 (1995), p. 1226.

[8] Pawel Romanczuk, Markus Bär, Werner Ebeling, Benjamin Lindner, and Lutz Schimansky-Geier. "Active brownian particles." In: The European Physical Journal Special Topics 202.1 (2012), pp. 1162.

[9] Fernando Peruani and Luis G Morelli. "Self-propelled particles with fluctuating speed and direction of motion in two dimensions." In: Physical review letters 99.1 (2007), p. 010602.

[10] Héctor Matías López, Jérémie Gachelin, Carine Douarche, Harold Auradou, and Eric Clément. "Turning bacteria suspensions into superfluids." In: Physical review letters 115.2 (2015), p. 028301.

[11] Christopher Dombrowski, Luis Cisneros, Sunita Chatkaew, Raymond E Goldstein, and John O Kessler. "Self-concentration and large-scale coherence in bacterial dynamics." In: Physical review letters 93.9 (2004), p. 098103.

[12] Elena O Budrene and Howard C Berg. "Dynamics of formation of symmetrical patterns by chemotactic bacteria." In: $\mathrm{Na}$ ture 376.6535 (1995), p. 49. 
[13] Eric Lauga, Willow R DiLuzio, George M Whitesides, and Howard A Stone. "Swimming in circles: motion of bacteria near solid boundaries." In: Biophysical journal 90.2 (2006), pp. 400412.

[14] Luanne Hall-Stoodley, J William Costerton, and Paul Stoodley. "Bacterial biofilms: from the natural environment to infectious diseases." In: Nature reviews microbiology 2.2 (2004), p. 95.

[15] Howard C Berg, Douglas A Brown, et al. "Chemotaxis in Escherichia coli analysed by three-dimensional tracking." In: $\mathrm{Na}$ ture 239.5374 (1972), pp. 500-504.

[16] Howard C Berg and Linda Turner. "Chemotaxis of bacteria in glass capillary arrays. Escherichia coli, motility, microchannel plate, and light scattering." In: Biophysical Journal 58.4 (1990), pp. 919-930.

[17] HANS MACHEMER. "Ciliary activity and the origin of metachrony in Paramecium: effects of increased viscosity." In: Journal of Experimental Biology 57.1 (1972), pp. 239-259.

[18] John R Blake and Michael A Sleigh. "Mechanics of ciliary locomotion." In: Biological Reviews 49.1 (1974), pp. 85-125.

[19] DM Woolley. "Motility of spermatozoa at surfaces." In: Reproduction 126.2 (2003), pp. 259-270.

[20] Ingmar H Riedel, Karsten Kruse, and Jonathon Howard. "A self-organized vortex array of hydrodynamically entrained sperm cells." In: Science 309.5732 (2005), pp. 300-303.

[21] WILLIAM G HAND and WOLFGANG HAUPT. "Flagellar activity of the colony members of Volvox aureus Ehrbg. during light stimulation." In: Journal of Eukaryotic Microbiology 18.3 (1971), pp. 361-364.

[22] Raymond E Goldstein. "Green algae as model organisms for biological fluid dynamics." In: Annual review of fluid mechanics 47 (2015).

[23] Niko Heddergott, Timothy Krüger, Sujin B Babu, Ai Wei, Erik Stellamanns, Sravanti Uppaluri, Thomas Pfohl, Holger Stark, and Markus Engstler. "Trypanosome motion represents an adaptation to the crowded environment of the vertebrate bloodstream." In: PLoS pathogens 8.11 (2012), e1003023.

[24] R Golestanian, TB Liverpool, and A Ajdari. "Designing phoretic micro-and nano-swimmers." In: New Journal of Physics 9.5 (2007), p. 126.

[25] Shashi Thutupalli, Ralf Seemann, and Stephan Herminghaus. "Swarming behavior of simple model squirmers." In: New Journal of Physics 13.7 (2011), p. 073021. 
[26] Maximilian Schmitt and Holger Stark. "Swimming active droplet: A theoretical analysis." In: EPL (Europhysics Letters) 101.4 (2013), p. 44008.

[27] Rémi Dreyfus, Jean Baudry, Marcus L Roper, Marc Fermigier, Howard A Stone, and Jérôme Bibette. "Microscopic artificial swimmers." In: Nature 437.7060 (2005), p. 862.

[28] Erik Gauger and Holger Stark. "Numerical study of a microscopic artificial swimmer." In: Physical Review E 74.2 (2006), p. 021907.

[29] Julien Deseigne, Sébastien Léonard, Olivier Dauchot, and Hugues Chate. "Vibrated polar disks: spontaneous motion, binary collisions, and collective dynamics." In: Soft Matter 8.20 (2012), pp. 5629-5639.

[30] Arshad Kudrolli, Geoffroy Lumay, Dmitri Volfson, and Lev S Tsimring. "Swarming and swirling in self-propelled polar granular rods." In: Physical review letters 100.5 (2008), p. 058001.

[31] Bradley J Nelson, Ioannis K Kaliakatsos, and Jake J Abbott. "Microrobots for minimally invasive medicine." In: Annual review of biomedical engineering 12 (2010), pp. 55-85.

[32] Debabrata Patra, Samudra Sengupta, Wentao Duan, Hua Zhang, Ryan Pavlick, and Ayusman Sen. "Intelligent, self-powered, drug delivery systems." In: Nanoscale 5.4 (2013), pp. 1273-1283.

[33] Wei Gao and Joseph Wang. "The environmental impact of micro/nanomachines: a review." In: Acs Nano 8.4 (2014), pp. 31703180.

[34] M Cristina Marchetti, Jean-François Joanny, Sriram Ramaswamy, Tanniemola B Liverpool, Jacques Prost, Madan Rao, and R Aditi Simha. "Hydrodynamics of soft active matter." In: Reviews of Modern Physics 85.3 (2013), p. 1143.

[35] Hugo Wioland, Francis G Woodhouse, Jörn Dunkel, John O Kessler, and Raymond E Goldstein. "Confinement stabilizes a bacterial suspension into a spiral vortex." In: Physical review letters 110.26 (2013), p. 268102.

[36] Jonathan R Howse, Richard AL Jones, Anthony J Ryan, Tim Gough, Reza Vafabakhsh, and Ramin Golestanian. "Self-motile colloidal particles: from directed propulsion to random walk." In: Physical review letters 99.4 (2007), p. 048102.

[37] Marco Polin, Idan Tuval, Knut Drescher, Jerry P Gollub, and Raymond E Goldstein. "Chlamydomonas swims with two "gears" in a eukaryotic version of run-and-tumble locomotion." In: Science 325.5939 (2009), pp. 487-490. 
[38] ME Cates and J Tailleur. "When are active Brownian particles and run-and-tumble particles equivalent? Consequences for motility-induced phase separation." In: EPL (Europhysics Letters) 101.2 (2013), p. 20010.

[39] Alexandre P Solon, ME Cates, and Julien Tailleur. "Active brownian particles and run-and-tumble particles: A comparative study." In: The European Physical Journal Special Topics 224.7 (2015), pp. 1231-1262.

[40] Knut Drescher, Kyriacos C Leptos, Idan Tuval, Takuji Ishikawa, Timothy J Pedley, and Raymond E Goldstein. "Dancing volvox: hydrodynamic bound states of swimming algae." In: Physical Review Letters 102.16 (2009), p. 168101.

[41] Nguyen HP Nguyen, Daphne Klotsa, Michael Engel, and Sharon C Glotzer. "Emergent collective phenomena in a mixture of hard shapes through active rotation." In: Physical review letters 112.7 (2014), p. 075701.

[42] Xiao-Lun $\mathrm{Wu}$ and Albert Libchaber. "Particle diffusion in a quasi-two-dimensional bacterial bath." In: Physical review letters 84.13 (2000), p. 3017.

[43] Pulak K Ghosh, Yunyun Li, Giampiero Marchegiani, and Fabio Marchesoni. Communication: Memory effects and active Brownian diffusion. 2015.

[44] Antoine Bricard, Jean-Baptiste Caussin, Nicolas Desreumaux, Olivier Dauchot, and Denis Bartolo. "Emergence of macroscopic directed motion in populations of motile colloids." In: Nature 503.7474 (2013), p. 95.

[45] Udo Erdmann and Werner Ebeling. "Collective motion of Brownian particles with hydrodynamic interactions." In: Fluctuation and Noise Letters 3.02 (2003), pp. L145-L154.

[46] Fernando Peruani, Andreas Deutsch, and Markus Bär. "Nonequilibrium clustering of self-propelled rods." In: Physical Review E 74.3 (2006), p. 030904.

[47] Khanh-Dang Nguyen Thu Lam, Michael Schindler, and Olivier Dauchot. "Self-propelled hard disks: implicit alignment and transition to collective motion." In: New Journal of Physics 17.11 (2015), p. 113056.

[48] Allison P Berke, Linda Turner, Howard C Berg, and Eric Lauga. "Hydrodynamic attraction of swimming microorganisms by surfaces." In: Physical Review Letters 101.3 (2008), p. 038102.

[49] Vasily Kantsler, Jörn Dunkel, Marco Polin, and Raymond E Goldstein. "Ciliary contact interactions dominate surface scattering of swimming eukaryotes." In: Proceedings of the National Academy of Sciences 110.4 (2013), pp. 1187-1192. 
[50] Guanglai Li and Jay X Tang. "Accumulation of microswimmers near a surface mediated by collision and rotational Brownian motion." In: Physical review letters 103.7 (2009), p. 078101.

[51] Jens Elgeti and Gerhard Gompper. "Wall accumulation of selfpropelled spheres." In: EPL (Europhysics Letters) 101.4 (2013), p. 48003 .

[52] Orsolya Sipos, K Nagy, R Di Leonardo, and P Galajda. "Hydrodynamic trapping of swimming bacteria by convex walls." In: Physical review letters 114.25 (2015), p. 258104.

[53] Saverio E Spagnolie, Gregorio R Moreno-Flores, Denis Bartolo, and Eric Lauga. "Geometric capture and escape of a microswimmer colliding with an obstacle." In: Soft Matter 11.17 (2015), pp. 3396-3411.

[54] A Kaiser, HH Wensink, and H Löwen. "How to capture active particles." In: Physical review letters 108.26 (2012), p. 268307.

[55] Alejandro Guidobaldi, Yogesh Jeyaram, Ivan Berdakin, Victor V Moshchalkov, CA Condat, Veronica I Marconi, Laura Giojalas, and AV Silhanek. "Geometrical guidance and trapping transition of human sperm cells." In: Physical Review E 89.3 (2014), p. 032720.

[56] Laura Restrepo-Pérez, Lluís Soler, Cynthia S Martínez-Cisneros, Samuel Sánchez, and Oliver G Schmidt. "Trapping self-propelled micromotors with microfabricated chevron and heart-shaped chips." In: Lab on a Chip 14.9 (2014), pp. 1515-1518.

[57] R Di Leonardo, L Angelani, D Dell'Arciprete, Giancarlo Ruocco, V Iebba, S Schippa, MP Conte, F Mecarini, F De Angelis, and E Di Fabrizio. "Bacterial ratchet motors." In: Proceedings of the National Academy of Sciences 107.21 (2010), pp. 9541-9545.

[58] Giorgio Volpe, Giovanni Volpe, and Sylvain Gigan. "Brownian motion in a speckle light field: tunable anomalous diffusion and selective optical manipulation." In: Scientific reports 4 (2014), p. 3936.

[59] Sungsu Park, Hyejin Hwang, Seong-Won Nam, Fernando Martinez, Robert H Austin, and William S Ryu. "Enhanced Caenorhabditis elegans locomotion in a structured microfluidic environment." In: PloS one 3.6 (2008), e2550.

[6o] Alexandre Morin, David Lopes Cardozo, Vijayakumar Chikkadi, and Denis Bartolo. "Diffusion, subdiffusion, and localization of active colloids in random post lattices." In: Physical Review E 96.4 (2017), p. 042611.

[61] Alexandre Morin, Nicolas Desreumaux, Jean-Baptiste Caussin, and Denis Bartolo. "Distortion and destruction of colloidal flocks in disordered environments." In: Nature Physics 13.1 (2017), p. 63. 
[62] C Reichhardt and CJ Olson Reichhardt. "Active matter transport and jamming on disordered landscapes." In: Physical Review E 90.1 (2014), p. 012701.

[63] Charles Reichhardt and Cynthia J Olson Reichhardt. "Absorbing phase transitions and dynamic freezing in running active matter systems." In: Soft Matter 10.38 (2014), pp. 7502-7510.

[64] Oleksandr Chepizhko, Eduardo G Altmann, and Fernando Peruani. "Optimal noise maximizes collective motion in heterogeneous media." In: Physical review letters 110.23 (2013), p. 238101.

[65] Amir Nourhani, Vincent H Crespi, and Paul E Lammert. "Guiding chiral self-propellers in a periodic potential." In: Physical review letters 115.11 (2015), p. 118101.

[66] Aparna Baskaran and M Cristina Marchetti. "Enhanced diffusion and ordering of self-propelled rods." In: Physical Review Letters 101.26 (2008), p. 268101.

[67] J Tailleur and ME Cates. "Statistical mechanics of interacting run-and-tumble bacteria." In: Physical review letters 100.21 (2008), p. 218103.

[68] Yaouen Fily and M Cristina Marchetti. "Athermal phase separation of self-propelled particles with no alignment." In: Physical review letters 108.23 (2012), p. 235702.

[69] Gabriel S Redner, Michael F Hagan, and Aparna Baskaran. "Structure and dynamics of a phase-separating active colloidal fluid." In: Physical review letters 110.5 (2013), p. 055701.

[70] Julian Bialké, Hartmut Löwen, and Thomas Speck. "Microscopic theory for the phase separation of self-propelled repulsive disks." In: EPL (Europhysics Letters) 103.3 (2013), p. 30008.

[71] Ivo Buttinoni, Julian Bialké, Felix Kümmel, Hartmut Löwen, Clemens Bechinger, and Thomas Speck. "Dynamical clustering and phase separation in suspensions of self-propelled colloidal particles." In: Physical review letters 110.23 (2013), p. 238301.

[72] I Theurkauff, C Cottin-Bizonne, J Palacci, C Ybert, and L Bocquet. "Dynamic clustering in active colloidal suspensions with chemical signaling." In: Physical review letters 108.26 (2012), p. 268303.

[73] Jeremie Palacci, Stefano Sacanna, Asher Preska Steinberg, David J Pine, and Paul M Chaikin. "Living crystals of light-activated colloidal surfers." In: Science 339.6122 (2013), pp. 936-940.

[74] Julian Bialké, Thomas Speck, and Hartmut Löwen. “Crystallization in a dense suspension of self-propelled particles." In: Physical review letters 108.16 (2012), p. 168301.

[75] G Briand and Olivier Dauchot. "Crystallization of Self-Propelled Hard Discs." In: Physical review letters 117.9 (2016), p. o98004. 
[76] Andreas M Menzel, Takao Ohta, and Hartmut Löwen. "Active crystals and their stability." In: Physical Review E 89.2 (2014), p. 022301.

[77] Roland Thar and Michael Kühl. "Complex pattern formation of marine gradient bacteria explained by a simple computer model." In: FEMS microbiology letters 246.1 (2005), pp. 75-79.

[78] Andreas M Menzel and Hartmut Löwen. "Traveling and resting crystals in active systems." In: Physical review letters 110.5 (2013), p. 055702.

[79] Maria R D'Orsogna, Yao-Li Chuang, Andrea L Bertozzi, and Lincoln S Chayes. "Self-propelled particles with soft-core interactions: patterns, stability, and collapse." In: Physical review letters 96.10 (2006), p. 104302.

[8o] J Schwarz-Linek, C Valeriani, A Cacciuto, ME Cates, D Marenduzzo, AN Morozov, and WCK Poon. "Phase separation and rotor self-assembly in active particle suspensions." In: Proceedings of the National Academy of Sciences 109.11 (2012), pp. 40524057.

[81] A Suma, G Gonnella, D Marenduzzo, and E Orlandini. "Motilityinduced phase separation in an active dumbbell fluid." In: EPL (Europhysics Letters) 108.5 (2014), p. 56004.

[82] Clarion Tung, Joseph Harder, Chantal Valeriani, and Angelo Cacciuto. "Micro-phase separation in two dimensional suspensions of self-propelled spheres and dumbbells." In: Soft Matter 12.2 (2016), pp. 555-561.

[83] Alexander P Petroff, Xiao-Lun Wu, and Albert Libchaber. "Fastmoving bacteria self-organize into active two-dimensional crystals of rotating cells." In: Physical review letters 114.15 (2015), p. 158102.

[84] Jing Yan, Sung Chul Bae, and Steve Granick. "Rotating crystals of magnetic Janus colloids." In: Soft Matter 11.1 (2015), pp. 147153 .

[85] Zahra Mokhtari, Timo Aspelmeier, and Annette Zippelius. "Collective rotations of active particles interacting with obstacles." In: EPL (Europhysics Letters) 120.1 (2017), p. 14001.

[86] PG Saffman. "Brownian motion in thin sheets of viscous fluid." In: Journal of Fluid Mechanics 73.4 (1976), pp. 593-602.

[87] Andrea Fiege, Benjamin Vollmayr-Lee, and Annette Zippelius. "Anomalous velocity distributions in active Brownian suspensions." In: Physical Review E 88.2 (2013), p. 022138. 
[88] Joshua A Anderson, Chris D Lorenz, and Alex Travesset. "General purpose molecular dynamics simulations fully implemented on graphics processing units." In: Journal of Computational Physics 227.10 (2008), pp. 5342-5359.

[89] Jens Glaser, Trung Dac Nguyen, Joshua A Anderson, Pak Lui, Filippo Spiga, Jaime A Millan, David C Morse, and Sharon C Glotzer. "Strong scaling of general-purpose molecular dynamics simulations on GPUs." In: Computer Physics Communications 192 (2015), pp. 97-107.

[90] AD Maude. "Non-random distribution of bull spermatozoa in a drop of sperm suspension." In: Nature 200.4904 (1963), pp. 381-381.

[91] Knut Drescher, Jörn Dunkel, Luis H Cisneros, Sujoy Ganguly, and Raymond E Goldstein. "Fluid dynamics and noise in bacterial cell-cell and cell-surface scattering." In: Proceedings of the National Academy of Sciences 108.27 (2011), pp. 10940-10945.

[92] Daisuke Takagi, Jérémie Palacci, Adam B Braunschweig, Michael J Shelley, and Jun Zhang. "Hydrodynamic capture of microswimmers into sphere-bound orbits." In: Soft Matter 10.11 (2014), pp. $1784-1789$.

[93] Mehdi Molaei, Michael Barry, Roman Stocker, and Jian Sheng. "Failed escape: solid surfaces prevent tumbling of Escherichia coli." In: Physical review letters 113.6 (2014), p. 068103.

[94] Mark C van Loosdrecht, Johannes Lyklema, Willem Norde, and AJ Zehnder. "Influence of interfaces on microbial activity." In: Microbiological reviews 54.1 (1990), pp. 75-87.

[95] Christophe Beloin, Agnès Roux, and J-M Ghigo. “Escherichia coli biofilms." In: Bacterial Biofilms. Springer, 2008, pp. 249-289.

[96] Jacinta C Conrad. "Physics of bacterial near-surface motility using flagella and type IV pili: implications for biofilm formation." In: Research in microbiology 163.9 (2012), pp. 619-629.

[97] Enkeleida Lushi, Vasily Kantsler, and Raymond E Goldstein. "Scattering of biflagellate microswimmers from surfaces." In: Physical Review E 96.2 (2017), p. 023102.

[98] Marlin U Thomas. "Some mean first-passage time approximations for the Ornstein-Uhlenbeck process." In: Journal of Applied Probability 12.3 (1975), pp. 600-604.

[99] Larbi Alili, P Patie, and Jesper Lund Pedersen. "Representations of the first hitting time density of an Ornstein-Uhlenbeck process 1." In: Stochastic Models 21.4 (2005), pp. 967-980. 
[10o] Susanne Ditlevsen and Ove Ditlevsen. "Parameter estimation from observations of first-passage times of the Ornstein-Uhlenbeck process and the Feller process." In: Probabilistic Engineering Mechanics 23.2 (2008), pp. 170-179.

[101] Konstantin Schaar, Andreas Zöttl, and Holger Stark. "Detention times of microswimmers close to surfaces: influence of hydrodynamic interactions and noise." In: Physical review letters 115.3 (2015), p. 038101.

[102] TW Marshall and EJ Watson. "A drop of ink falls from my pen... it comes to earth, i know not when." In: Journal of Physics A: Mathematical and General 18.18 (1985), p. 3531.

[103] Matteo Contino, Enkeleida Lushi, Idan Tuval, Vasily Kantsler, and Marco Polin. "Microalgae scatter off solid surfaces by hydrodynamic and contact forces." In: Physical review letters 115.25 (2015), p. 258102.

[104] Jan Groenewold and Willem K Kegel. "Anomalously large equilibrium clusters of colloids." In: The Journal of Physical Chemistry B 105.47 (2001), pp. 11702-11709.

[105] Anna Stradner, Helen Sedgwick, Frédéric Cardinaux, Wilson CK Poon, Stefan U Egelhaaf, and Peter Schurtenberger. "Equilibrium cluster formation in concentrated protein solutions and colloids." In: Nature 432.7016 (2004), pp. 492-495.

[106] Andrew I Campbell, Valerie J Anderson, Jeroen S van Duijneveldt, and Paul Bartlett. "Dynamical arrest in attractive colloids: The effect of long-range repulsion." In: Physical review letters 94.20 (2005), p. 208301.

[107] Daniel Ben-Avraham and Shlomo Havlin. Diffusion and reactions in fractals and disordered systems. Cambridge university press, 2000.

[108] Felix Höfling, Tobias Munk, Erwin Frey, and Thomas Franosch. "Critical dynamics of ballistic and Brownian particles in a heterogeneous environment." In: The Journal of chemical physics 128.16 (2008), p. 164517.

[109] Markus Spanner, Felix Höfling, Gerd E Schröder-Turk, Klaus Mecke, and Thomas Franosch. "Anomalous transport of a tracer on percolating clusters." In: Journal of Physics: Condensed Matter 23.23 (2011), p. 234120.

[110] Bong June Sung and Arun Yethiraj. "Lateral diffusion and percolation in membranes." In: Physical review letters 96.22 (2006), p. 228103 .

[111] Maria Zeitz, Katrin Wolff, and Holger Stark. "Active Brownian particles moving in a random Lorentz gas." In: The European Physical Journal E 40.2 (2017), p. 23. 
[112] Alexandre Morin, David Lopes Cardozo, Vijayakumar Chikkadi, and Denis Bartolo. "Self-propulsion through disorder: localization transition in active Lorentz gases." In: arXiv preprint arXiv:1702.07655 (2017).

[113] Teresa Bauer, Felix Höfling, Tobias Munk, Erwin Frey, and Thomas Franosch. "The localization transition of the two-dimensional Lorentz model." In: The European Physical Journal Special Topics 189.1 (2010), pp. 103-118.

[114] Felix Höfling, Thomas Franosch, and Erwin Frey. "Localization transition of the three-dimensional Lorentz model and continuum percolation." In: Physical review letters 96.16 (2006), p. 165901.

[115] Simon K Schnyder, Markus Spanner, Felix Höfling, Thomas Franosch, and Jürgen Horbach. "Rounding of the localization transition in model porous media." In: Soft Matter 11.4 (2015), pp. 701-711.

[116] J Harder, SA Mallory, C Tung, C Valeriani, and A Cacciuto. "The role of particle shape in active depletion." In: The Journal of Chemical Physics 141.19 (2014), p. 194901.

[117] Joseph Harder, Chantal Valeriani, and Angelo Cacciuto. "Activityinduced collapse and reexpansion of rigid polymers." In: Physical Review E 90.6 (2014), p. 062312.

[118] Andreas Kaiser and Hartmut Löwen. "Unusual swelling of a polymer in a bacterial bath." In: The Journal of chemical physics 141.4 (2014), p. 044903.

[119] Laura Mely Ramírez, Charles A Michaelis, Javier E Rosado, Elias K Pabon, Ralph H Colby, and Darrell Velegol. "Polloidal chains from self-assembly of flattened particles." In: Langmuir 29.33 (2013), pp. 10340-10345.

[120] Yuji Sasaki, Yoshinori Takikawa, Venkata SR Jampani, Hikaru Hoshikawa, Takafumi Seto, Christian Bahr, Stephan Herminghaus, Yoshiki Hidaka, and Hiroshi Orihara. "Colloidal caterpillars for cargo transportation." In: Soft Matter 10.44 (2014), pp. 8813-8820. 


\section{DECLARATION}

I declare that this thesis was composed by myself, and that this work has not been submitted for any other degree or professional qualification except as specified.

Göttingen, 2018

Zahra Mokhtari 



\section{COLOPHON}

This document was typeset using the typographical look-and-feel classicthesis developed by André Miede. The style was inspired by Robert Bringhurst's seminal book on typography "The Elements of Typographic Style". classicthesis is available for both LTEX and LYX:

https://bitbucket.org/amiede/classicthesis/

Happy users of classicthesis usually send a real postcard to the author, a collection of postcards received so far is featured here:

http://postcards . miede. de/

Final Version as of September 4, 2018 (classicthesis). 